\title{
An fMRI study of chromatic processing in humans
}

\author{
Spatial and temporal characteristics \\ of the cortical visual areas
}

\begin{abstract}
PhD Thesis
in partial fulfillment of the requirements for the degree

"Doctor of Philosophy (PhD)/Dr. rer. nat."

in the Center for Systems Neuroscience Graduate Program

Faculty of Biology, Georg-August-Universität Göttingen
\end{abstract}

Submitted by

DANY V. D'SOUZA

Göttingen, 2009 
Doctoral thesis committee:

External thesis advisor:

Date of submission of the thesis:

Date of disputation:
Prof. Dr. Jens Frahm (Advisor, First Referee) Biomedizinische NMR Forschungs $\mathrm{GmbH}$ am Max-Planck-Institut für biophysikalische Chemie Am Fassberg 11

37077 Göttingen

Prof. Dr. Barry B. Lee (Advisor)

SUNY - State College of Optometry Biological Sciences

33 West 42nd Street, New York, NY 10036

Prof. Dr. Stefan Treue (Second Referee) Abt. Kognitive Neurowissenschaften Deutsches Primatenzentrum (DPZ) Kellnerweg 4

37077 Göttingen

Prof. Dr. Hans Strasburger

Abt. Medizinische Psychologie

Universität Göttingen

Waldweg 37

37073 Göttingen

22 July, 2009

09 September, 2009 
I hereby declare that this thesis has been written independently and with no other sources and aids than quoted.

Göttingen, 22 July, 2009

Dany V. D'Souza 

For my beloved parents 



\section{Contents}

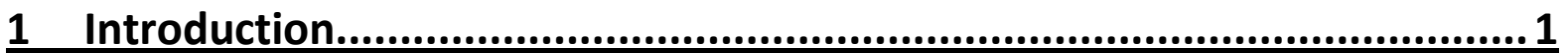

1.1 The organization of the visual system ………

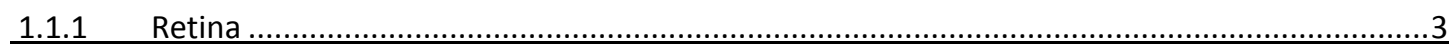

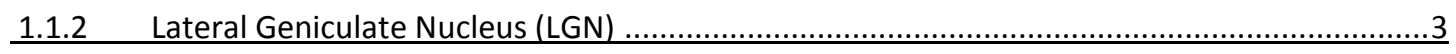

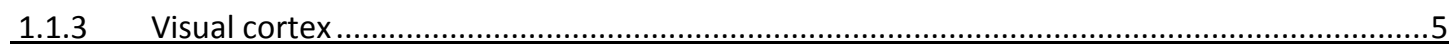

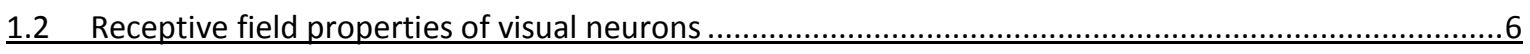

1.2.1 Receptive fields of retinal ganglion and LGN cells ....................................................

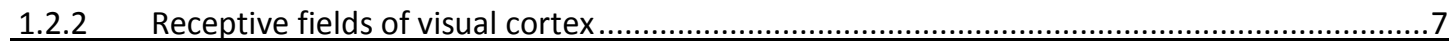

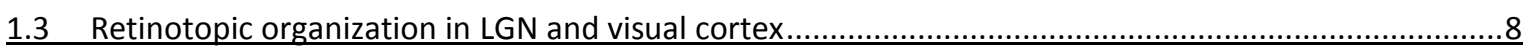

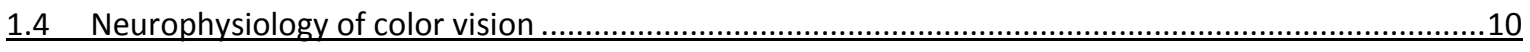

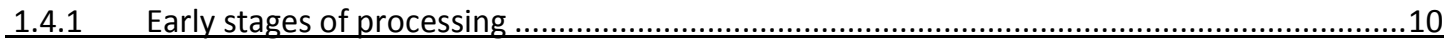

Spectral coding by cone photoreceptors in the retina .............................................10

Spectral opponency in retinal ganglion cells ..............................................................12

Segregation and spectral opponency in LGN cells.......................................................14

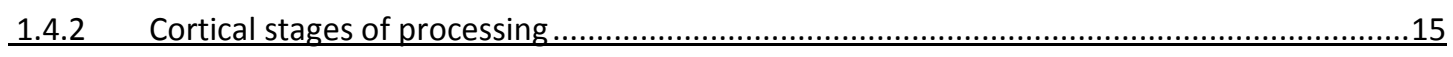

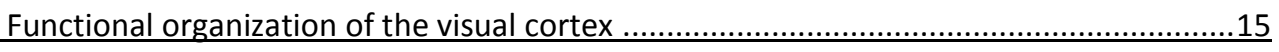

Chromatic properties of V1 receptive fields ................................................................16

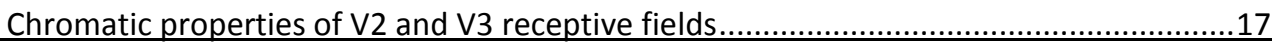

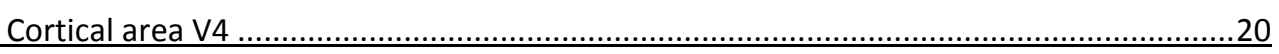

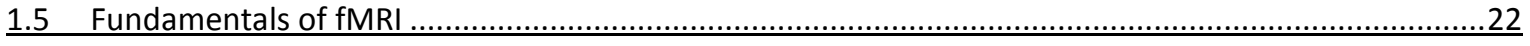

1.5.1 Basic principles of magnetic resonance imaging (MRI) ………………………………....22

1.5.2 Blood oxygenation level dependent (BOLD) fMRI .......................................................24

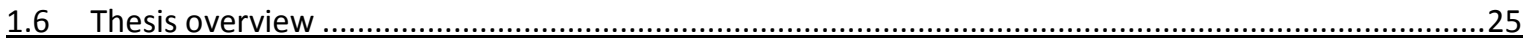

1.6.1 Color discrimination deteriorates across the visual field ...................................................25

1.6.2 Eccentricity-dependent chromatic responses in human visual cortex .................................28

1.6.3 Psychophysical chromatic contrast sensitivity deteriorates at high temporal modulation frequencies ................................................................................

1.6.4 Cortical responses to temporal frequency-dependent chromatic information......................33

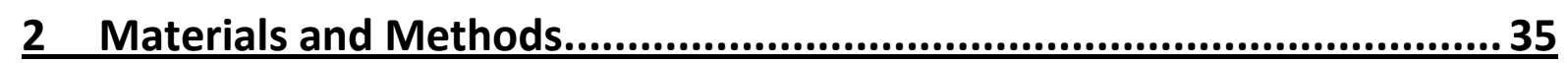

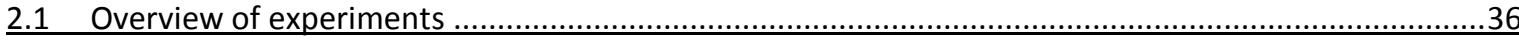

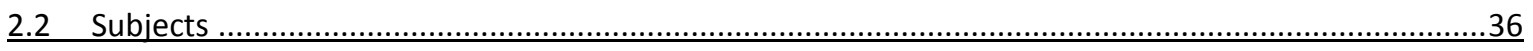

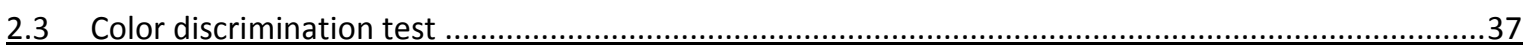

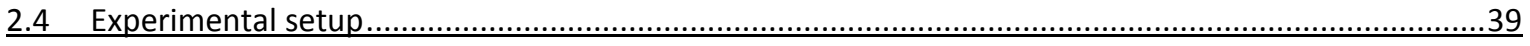




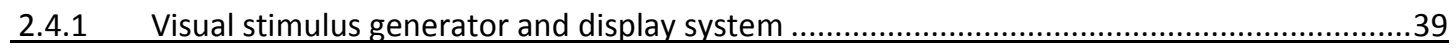

2.4.2 Calibration of the LCD projector ……………….......................................................40

2.5 Retinotopic mapping experiments …………………

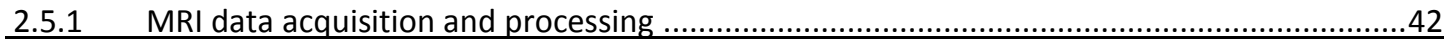

2.5.2 Visual stimuli, experimental design, and data analysis .....................................................44

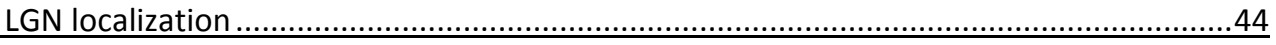

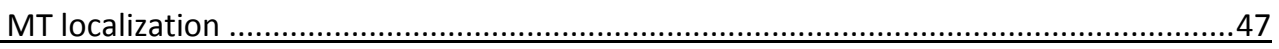

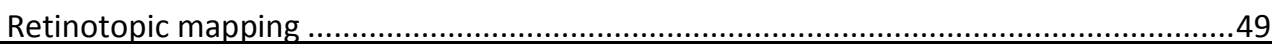

2.6 Definition of the chromatic and luminance pathway selective stimuli ………………………………...56

2.6.1 L-M cone-opponent (red-green) pathway selective stimulus ............................................59

2.6.2 L+M (luminance) pathway selective stimulus ……………………………………………..61

2.6.3 S-cone (blue-yellow) pathway selective stimulus ............................................................62

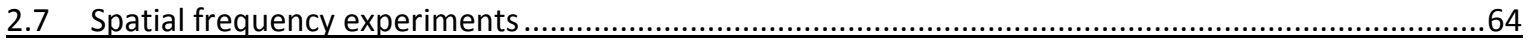

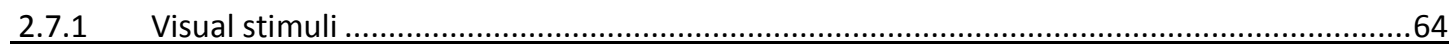

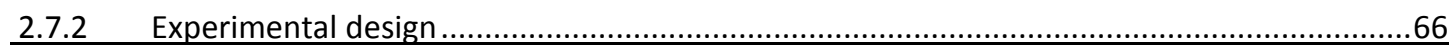

2.7.3 fMRI measurements and data processing ..................................................................68

2.7.4 Eccentricity-based region-of-interest analysis ...................................................................69

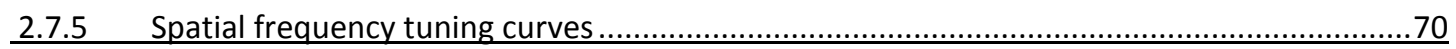

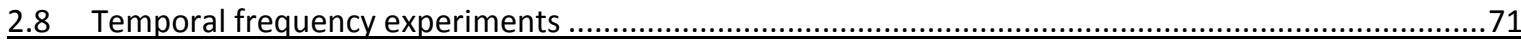

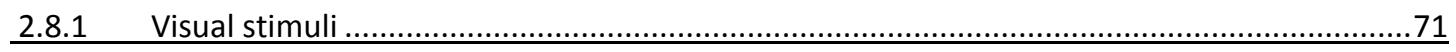

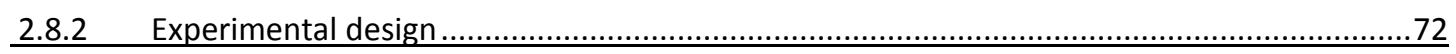

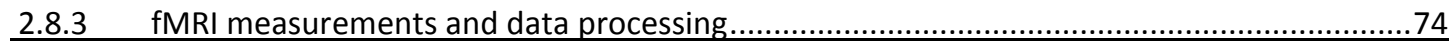

2.8.4 Visual area based region-of-interest analysis ...................................................................74

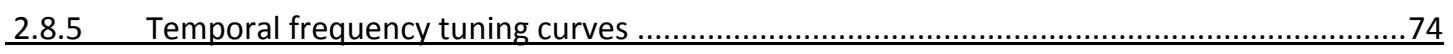

2.8.6 Cluster analysis of visual areas .........................................................................................75

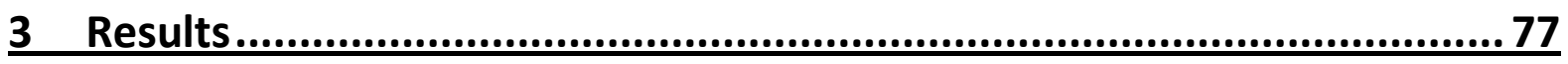

3.1 Dependence of chromatic and luminance responses on visual field eccentricity

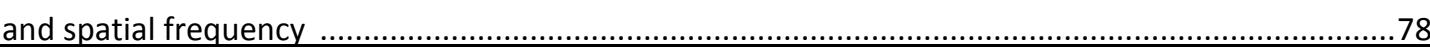

3.1.1 Sensitivity as a function of visual field eccentricity in V1 .................................................78

3.1.2 Sensitivity as a function of spatial frequency across visual field

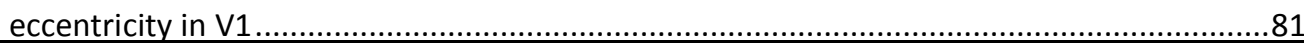

3.1.3 Sensitivity as a function of visual field eccentricity in extrastriate visual areas (V2v, VP, and V4) ……………………………............................................

3.2 Temporal frequency tuning of chromatic and luminance responses in human

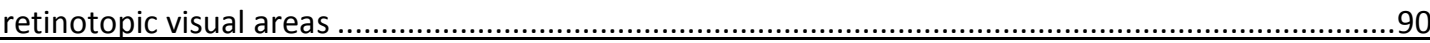

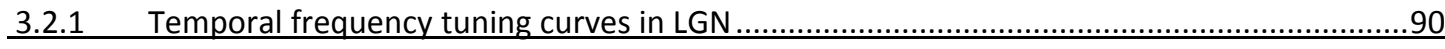

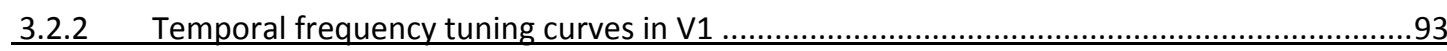

3.2.3 Temporal frequency tuning curves in ventral visual areas: V2v, VP, and V4 ........................96

3.2.4 Temporal frequency tuning curves in dorsal visual areas: V2d, V3d, V3a, and MT ..............99

3.2.5 Summary of temporal frequency tuning curves ..............................................................102

3.2.6 Cluster analysis of visual areas ......................................................................................103 


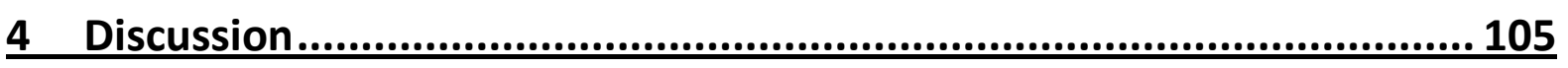

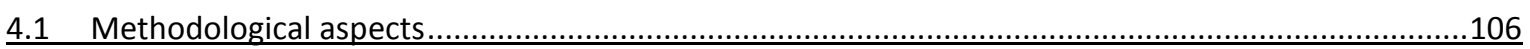

4.2 Dependence of chromatic and luminance responses on visual field eccentricity and spatial frequency …….......................................................................................................108

4.2.1 Psychophysical sensitivity loss to peripheral chromatic modulation does not have a neural correlate in V1 ...................................................................................108

4.2.2 Interaction of the stimulus spatial frequency with eccentricity-dependent responses in V1 ..............................................................................................................109

4.2.3 Does eccentricity-dependent psychophysical chromatic sensitivity loss have neural correlates in the extrastriate visual areas?

4.3 Temporal frequency tuning of chromatic and luminance responses in human retinotopic visual areas .114

4.3.1 Does high temporal frequency chromatic information pass through LGN? ......................114

4.3.2 V1 filters high temporal frequency blue-yellow chromatic information .............................117

4.3.3 Temporal frequency tuning in extrastriate visual areas..................................................118

4.3.4 Do 'Slow' and 'fast' information processing streams exist in the visual cortex? ..................120

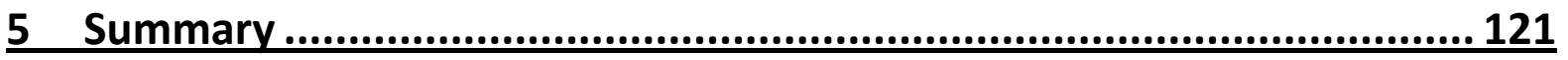

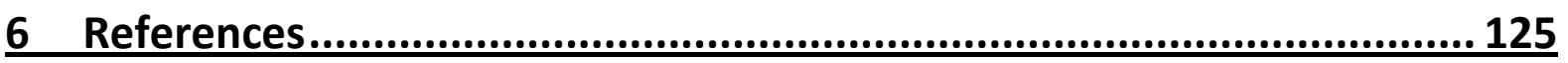

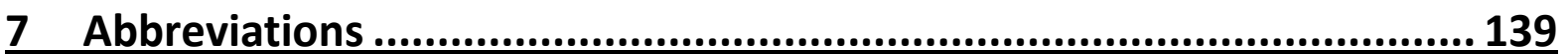

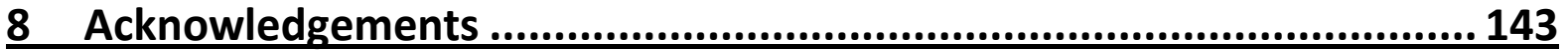

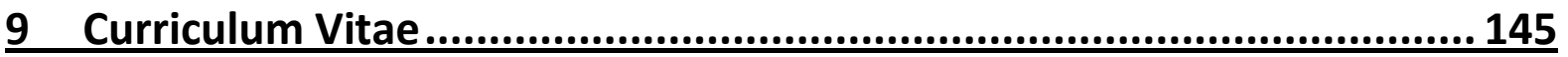


Introduction 
"To a large extent, we conceive of the cerebral cortex, or at least the sensory part of it, as an organ that represents the external world in some form of code. We thus speak of the visual cortex as a "cortical retina", involved in "analyzing" or "representing" the visual world and of "interpreting" the visual image that is formed on the retina. It is not surprising to find therefore that, in effort to understand how this retinal image is formed, most of the physiological work on color vision has concentrated on the retina and most of the perceptual work has been designed and interpreted in retinal terms. We have obtained a great deal of important and interesting information as a result. But in the last few years, the emphasis has started to shift to the cortex. With this shift has come a more general realization that color vision is a much elaborate process, requiring the cortex to undertake complex operations to construct colors."

Semir Zeki, 1990

This thesis attempts to elucidate the human cortical representation of chromatic information in the intermediate periphery of the visual field using functional magnetic resonance imaging (fMRI). Similarly, it also aims to investigate how different cortical visual areas respond to temporal modulation of chromatic fields. In this introductory chapter, an overview of scientific background pertaining to this thesis is provided. The organization of the primate visual system as well as the neurophysiological basis of color vision are described in the early sections of the chapter, followed by a brief introduction to blood oxygenation level dependent (BOLD) fMRI. Finally, the research questions are addressed in the thesis overview section. 


\subsection{The organization of the visual system}

\subsubsection{Retina}

In primates, the path for visual information processing begins with light absorption in a dense mosaic of light-sensitive cells, the rod and the cone photoreceptors. The photoreceptors are in the deepest layers of the retina, next to the pigment epithelium, and the light must pass through rows of other, transparent cell layers (ganglion-, amacrine-, bipolar-, and horizontal-cells) before reaching the receptors (Figure 1.1). Functionally, the retina consists of these five main cell types, and in the retinal transmission of information the receptors are the first elements and ganglion cells are the last. The ganglion cells project to higher brain centers via their axons that form between 1-2 million nerve fibers. The optic nerve leaves the eye at about 17 deg to the nasal side of the fovea. These are the nerve bundles that form the connections between the ganglion cells in the retina and the cells in the lateral geniculate nucleus (LGN), the relay station in the pathway to higher brain centers.

\subsubsection{Lateral Geniculate Nucleus (LGN)}

The LGN constitutes the main relay station for visual information from retina to cortex. Approximately $90 \%$ of the retinal ganglion cells project to the LGN, while the remainder connect to the superior colliculi and the pulvinar. The LGN in each thalamic hemisphere is distinguishable by its 6 distinct layers (Figure 1.1). The two ventral layers (layers 1 and 2) consist of relatively large magnocellular neurons, while the four dorsal layers (3 through 6) contain relatively small parvocellular neurons. In layers intercalated between the parvo- and magnocellular layers are some granular neurons, called koniocellular neurons. Because retinal fibers of the nasal retina cross over in the optic chiasm, each LGN receives visual information stemming from the contra-lateral visual field. For instance, the left LGN receives information from the left halves of each retina, which sample the right visual field. Each layer in LGN receives input from only one eye; layers 2, 3 and 5 from the eye on the same side as the LGN, and layers 1, 4 and 6 from the eye on the opposite side. 


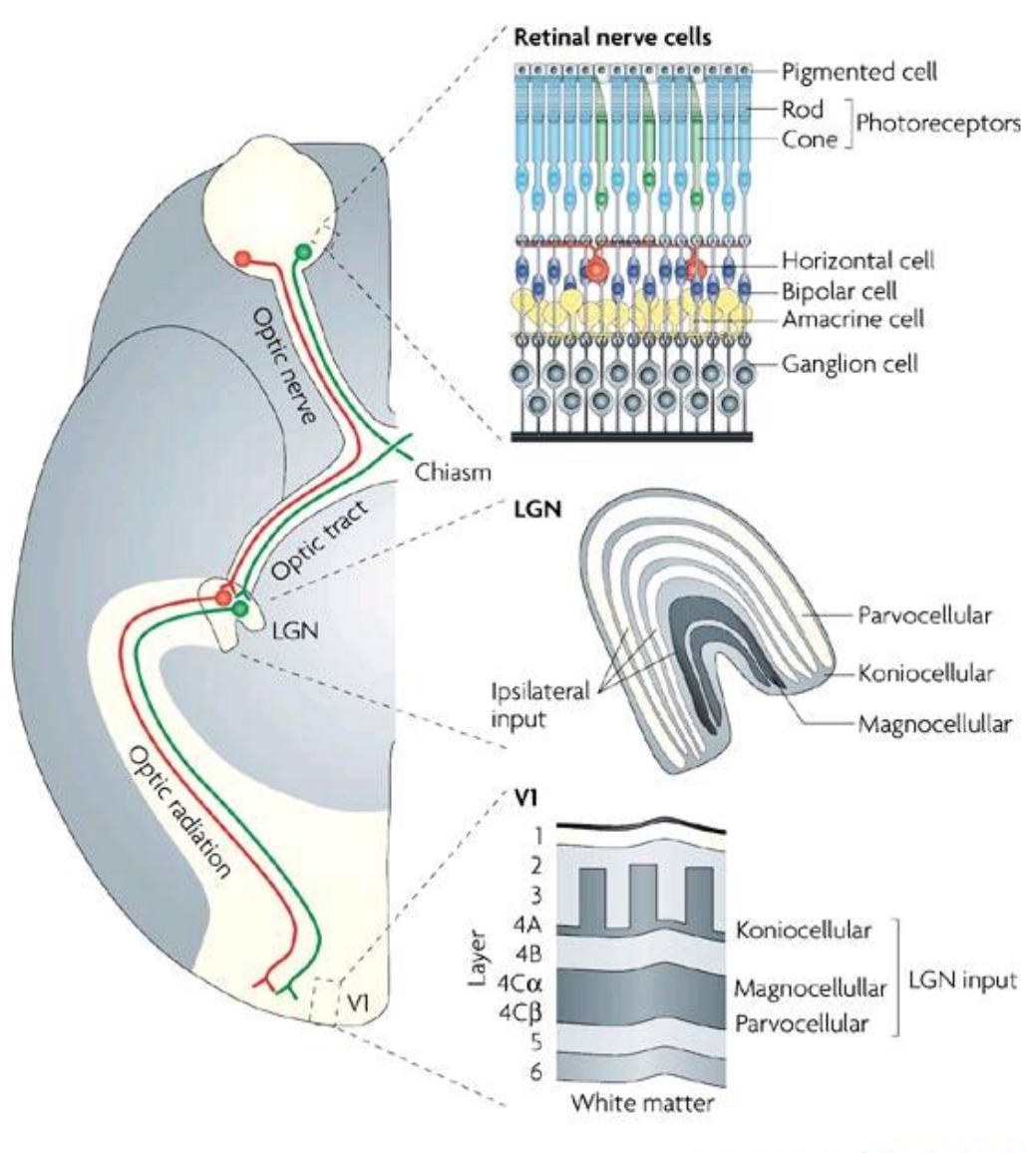

Nature Reviews | Neuroscience

Figure 1.1: The dominant visual pathways in primates

The left panel shows a schematic drawing of the pathway from the retina to the primary visual cortex (V1) through the dorsal lateral geniculate nucleus (LGN) of the thalamus. The right panels highlight the important anatomical structures. Light entering the eye passes through the ganglion cells and is imaged on the photoreceptor layer (rod photoreceptors, which are not active in color vision, are found between the cones). Signals from photoreceptors pass through bipolar cells to ganglion cells, the axons of which form the optic nerve, which projects principally to the LGN. The horizontal and amacrine cell pathways within the retina allow spatial comparisons of cone signals. Ganglion cells from the temporal retina project to the ipsilateral LGN (red lines) and those from the nasal retina project to the contralateral LGN (green lines). Within the LGN, the projections from the two eyes are aligned, so the same topographic map (of the contralateral half of the visual field) is found in all layers. The axons of LGN neurons project almost exclusively to V1, where they terminate primarily in layer 4 and form ocular dominance columns (a small fraction of LGN cells project to extrastriate areas: see Sincich et al. (2004) and the references therein). The termination site within layer 4 depends on the layer in which the LGN neuron is found: parvocellular (PC) cells project mainly to layer $4 C \beta$, magnocellular (MC) to layer $4 \mathrm{C} \alpha$, and koniocellular (KC) cells to layer $4 \mathrm{~A}$ and lower layer 3 . The shading depicts the distinct pattern that emerges when slices through $\mathrm{V} 1$ are stained for cytochrome oxidase (CO) activity. Reactivity is particularly high in layer 4 and in patches that dot the superficial layers 2 and 3. Source: Solomon \& Lennie (2007). 


\subsubsection{Visual cortex}

An estimated $50-60 \%$ of the cortex is engaged in the processing of visual information. Thirtytwo anatomically distinct visual areas have been identified in macaque cortex, of which 25 are primarily visual and 7 are involved in visually-guided motor control (Felleman \& Essen, 1991; Merigan \& Maunsell, 1993; Werner). The optic radiation forwards the signals from LGN towards the primary visual cortex (V1) (Figure 1.1), situated in the calcarine sulcus of the occipital lobe of primates, and from V1 the information is subsequently distributed across several visual areas. It is believed that visual information is segregated into two main cortical streams, "what" and "where" streams (Ungerleider \& Mishkin, 1982; Goodale \& Milner, 1992), which seem to operate across distinct areas (Figure 1.2). The "what" stream (or temporal pathway) - traveling ventrally in the brain includes areas such as V2, VP, and V4 - is believed to be mainly associated with recognition of objects, a perceptual system, and thus with color-, pattern- and form-perception. The "where" stream (or parietal pathway) encompassing mainly the dorsal cortical areas such as V3, medial temporal (MT) cortex, and parietal areas - is assumed to be mostly associated with spatio-temporal information processing.
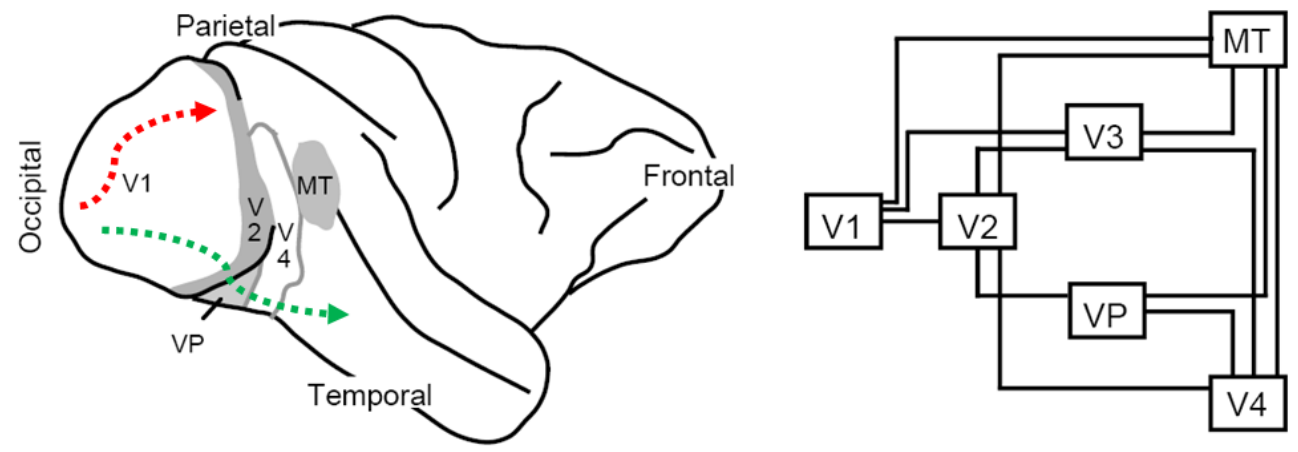

Figure 1.2: Visual streams and areas

Location of select visual areas (left panel) and the major lobes (occipital, parietal, temporal, frontal) of the right hemisphere of macaque cortex (lateral view). Cortical area V3 cannot be seen from this view. The parietal and temporal pathways are shown in red and green dashed lines, respectively. The schematic on the right shows some of the major connecting circuits among visual areas that constitute the "where" and "what" pathways. Areas V1, V2, V3, V4, ventral posterior (VP) and medial temporal (MT) are shown. Source: Modified after Werner J. S.; https://redwood.berkeley.edu/bruno/npb261b/werner-reading/Werner2.pdf. 


\subsection{Receptive field properties of visual neurons}

\subsubsection{Receptive fields of retinal ganglion and LGN cells}

The source of output from the retina to the rest of the brain is the action potentials arising from the ganglion cells. The inputs to a ganglion cell originate from neighboring photoreceptors in a circumscribed area of the retina (and a corresponding area in the visual field), the receptive field for that cell. In effect, the ganglion cell's receptive field is the area of the retina that the ganglion cell monitors. The receptive fields of ganglion cells have two important features. First, the receptive fields prove to be roughly circular. Second, in most ganglion cells the receptive field is divided into two parts: a circular zone at the center, called receptive field center, and the remaining area of the field, called the surround (Lee, 1996). Ganglion cells respond optimally to differential illumination of the receptive field center and surround (Figure 1.3).

Two classes of ganglion cells can be distinguished by their response to small spot of light applied to the center of their receptive field. ON-center ganglion cells are excited when light is directed to the center of their receptive field (Figure 1.3). Light applied to the surround inhibits the cell. OFF-center ganglion cells are inhibited by light applied to the center of their receptive field. Light excites an OFF-center ganglion cells when it is directed to the surround of the receptive field. In both types of cells the response evoked by a ring of light on the entire surround partially cancels the response evoked by light directed to the center. For this reason, diffuse illumination of the entire receptive field (ganzfeld stimulus) evokes a smaller response in either type of cell. The receptive fields of the LGN cells are essentially identical to ganglion cell receptive fields (Wiesel \& Hubel, 1966). 
a

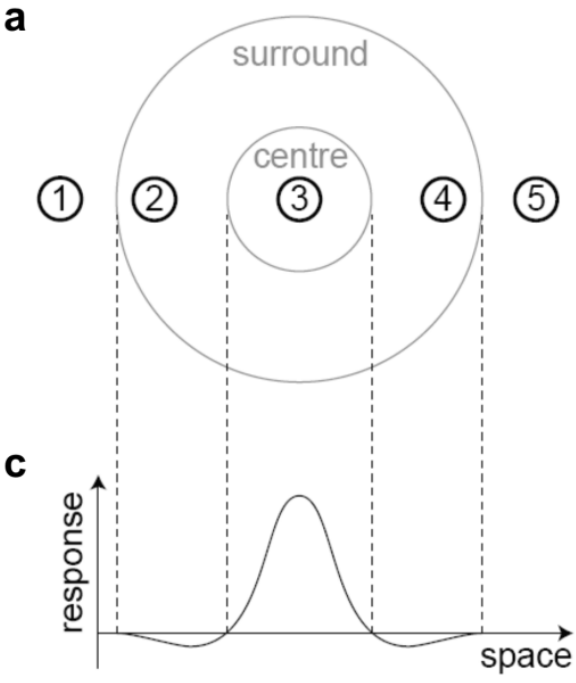

b

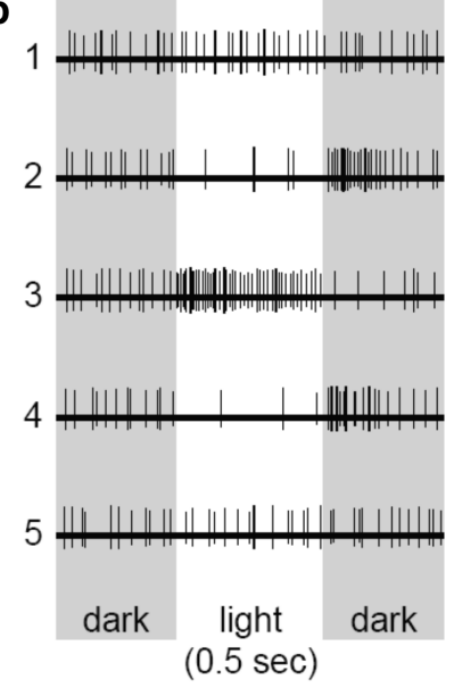

Figure 1.3: Center-surround receptive field structure of visual neurons

a) The receptive field of visual neurons consists of an excitatory center region, with a larger, concentric, inhibitory surround. The cell modeled here is an ON-center cell. An increment of light in the regions 1-5 will elicit the corresponding response shown in b). An increment of light at positions 1 or 5 causes no change in the firing rate of the cell, as both positions are outside the receptive field. Positions 2 and 4 fall within the inhibitory surround, and thus an increment of light at either of these positions will decrease the firing rate of the cell. Position 3 falls within the center region, and thus an increment of light at this position causes an increase in the firing rate of the cell. c) The spatial response of visual neurons can be modeled by a differenceof-gaussians (DOG) model, with a typical output shown here. Source: Adapted from Szmajda (2006).

\subsubsection{Receptive fields of visual cortex}

Unlike the simple center-surround receptive field organization of the ganglion and LGN cells, the receptive fields of cells of the primary visual cortex (striate cortex or V1) have more complex stimulus properties such as orientation and direction selectivity. Hubel and Wiesel (1962) classified cortical neurons into three types, namely simple-, complex-, and hypercomplex-cells, depending on their receptive field characteristics. The simple cells respond optimally to lines or bars having particular orientation and position in the visual field, and their receptive fields are elongated. Complex cells also respond optimally to lines or edges of a particular orientation, but are insensitive to the location or phase of the lightdark areas. The responses of some complex cells are enhanced by motion in a particular direction and speed. Hypercomplex cells respond to stripes and angles of a certain length, 
which move in the preferred direction across the receptive field (Hubel \& Wiesel, 1962). The elongated excitatory and inhibitory zones of simple and complex receptive fields are thought to emerge through combination of several concentrically organized cells. In the extrastriate visual areas, cells can have very large receptive fields requiring very complex images to excite the cell.

\subsection{Retinotopic organization in LGN and visual cortex}

The spatial position of the ganglion cells within the retina is preserved by the spatial organization of the neurons within the LGN layers. The back of the LGN contains neurons whose receptive fields are near fovea. Toward front of the nucleus, the receptive field locations become increasingly peripheral. This spatial layout is called retinotopic organization because of the topological organization of the receptive fields in the LGN parallels the organization in the retina. The neurons in area V1 are also retinotopically (visuotopically) arranged.

V1 contains an ordered map of the visual field. Each hemifield is represented on the contralateral hemisphere. On each side, the upper quadrants are represented below the calcarine sulcus, while the lower quadrants are represented above the calcarine sulcus. In each hemisphere, a disproportionately large anatomical area of the visual cortex is devoted to the representation of the fovea, which is the retinal area of greatest acuity (Figure 1.4). The connections between V1 and extrastriate areas, and among extrastiate areas, are also topographically organized; thus, neurons in the extrastriate area form a separate map of the visual field. In fMRI experiments, visual field topography is used to identify and map visual areas (Sereno et al., 1994; Sereno et al., 1995; DeYoe et al., 1996; Wandell, 1999). Mapping between the retina and the cortex can be best described as a log-polar transformation, in which standard axes in the retina are transformed into polar axes in the cortex: eccentricity (distance from fovea) and polar angle (angle from horizontal axis). In order to determine the topographical cortical representations in $\mathrm{fMRI}$ experiments, subjects are usually stimulated at selected locations while fixating on a central fixation cross. Mapping the angle component of the retinotopic map reveals multiple horizontal and vertical meridian 
representations arranged in approximately parallel bands along the cortical surface. These vertical and horizontal meridian representations alternate and define the borders between mirror-symmetric retinotopic areas. Perpendicular to these bands lie iso-eccentricity bands, which constitute an eccentricity gradient. As mentioned earlier, the representation of the fovea is greatly expanded compared to the representation of the periphery.

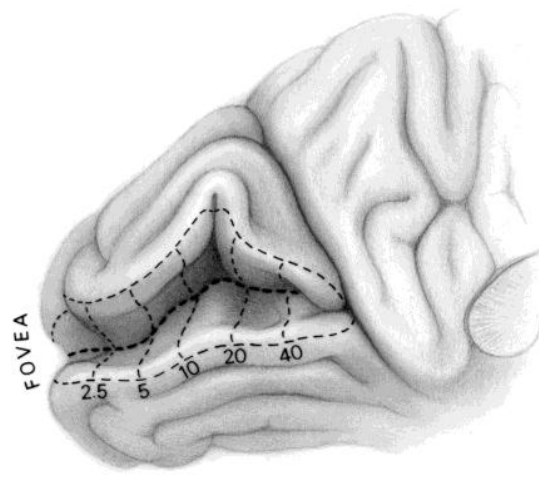

LEFT VISUAL CORTEX

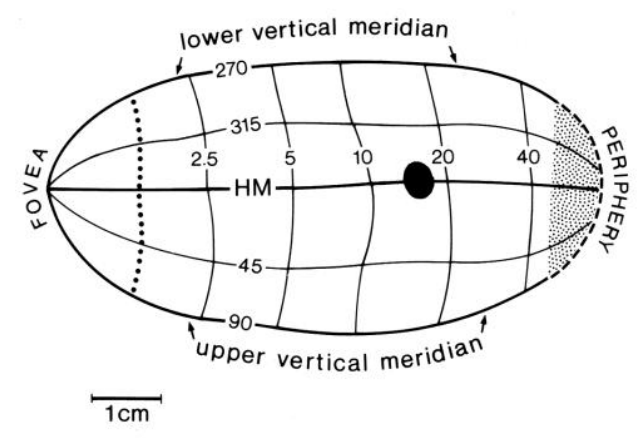

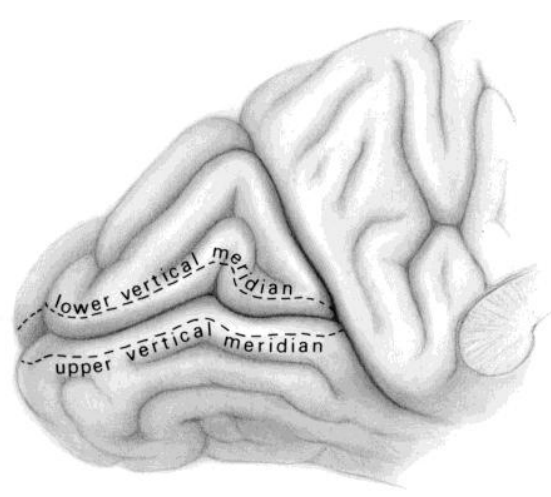

RIGHT VISUAL FIELD

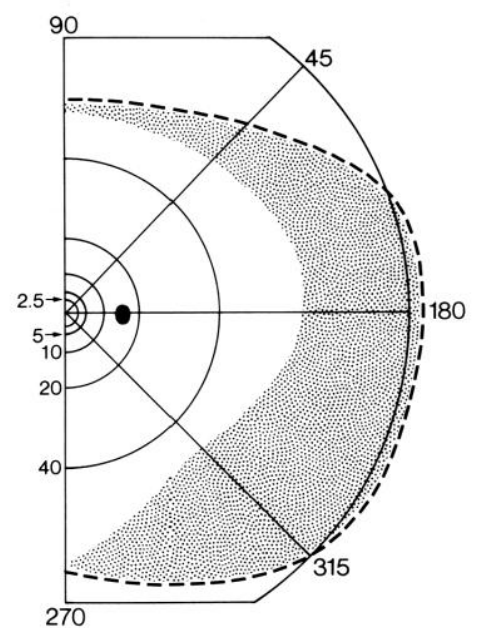

Figure 1.4: Retinotopic map of the human striate cortex

Upper right shows left occipital lobe, with most of striate cortex buried in the calcarine fissure. Upper left shows the fissure opened, with distance (eccentricity) from the fovea (center of gaze) marked in degrees. The horizontal meridian (HM) runs roughly along the base of the fissure. Lower left shows the map, removed from the calcarine fissure and flattened artificially. Dots depict occipital pole; the central 1 deg is located on the exposed lateral convexity, although this varies from person to person. Note the immense magnification of central vision. Dark oval $=$ blind spot, stippled zone $=$ monocular crescent.

Source: http://vision.ucsf.edu/hortonlab/ResearchProgram\%20Pics/retinotopicMap.jpg. 


\subsection{Neurophysiology of color vision}

Perception of color, of which underlying various neurophysiological processes occur at different stages along the visual pathway, is initiated by light falling on the retina. In the primate visual system, color information is considered to be processed at three different stages. First, at the retinal stage, light energy is transformed into L-, M-, and S-cone signals. The retinal ganglion cells and cells of LGN constitute the second stage of processing. Here, the L-, M-, and S-cone signals are linearly combined (either added or subtracted), forming cell systems for three parallel pathways which carry red-green, luminance and blue-yellow information about the visual input. The parallel pathways have projections in the primary visual cortex (V1). Subsequently, from here the information is distributed to extrastriate visual areas for higher-order processing. An association of cortical visual areas constitutes the third stage of color information processing. The physiological properties of each stage of color processing are described in the following sections.

\subsubsection{Early stages of processing}

\section{Spectral coding by cone photoreceptors in the retina}

Humans with normal color vision have three cone types, each type with different pigment and spectral (de Monasterio et al., 1981; Lennie, 2000) sensitivity. A cone photoreceptor is named after the spectral region it covers: L-, M-, and S-cones for long-, middle-, and shortwavelength sensitive receptors. The spectral sensitivities of the cones can be seen in Figure 1.5 (Stockman et al., 2000). The S-cone type is sensitive to shorter wavelengths and has its peak sensitivity around $440 \mathrm{~nm}$ (commonly referred to as "blue (B)-cone"). The M-cone is sensitive to the middle wavelengths of the spectrum, peaking around $535 \mathrm{~nm}$ (the "green (G)-cone"). Finally, the L-cone is sensitive to longer wavelengths, peaking around $565 \mathrm{~nm}$ (the "red(R)-cone") (Schnapf \& Baylor, 1987). The number and density of each cone class varies across the retinal receptor mosaic. The S-cones amount $5-10 \%$ in the human retina. The ratio of L- and M-cones varies among individuals (Brainard et al., 2000; Hofer et al., 2005). The region of highest acuity in human retina is called the fovea, in which receptor 
density is highest. The center fovea contains only $\mathrm{L}-$ and $\mathrm{M}$ - and no S-cones. The $\mathrm{L}-$ and $\mathrm{M}$ cones in the fovea are slender, optimizing their ability to sample small areas of visual space.

Although the relative excitation of cones cannot be directly associated with the color perceived, the three different cone types make trichromatic color vision, i.e. color matches with three variables, possible. If one type is lacking, for instance the L- or the M-cones, the result is color vision with only two variables, called dichromacy. This leads to color confusions, most commonly in a failure to distinguish between red and green colors.

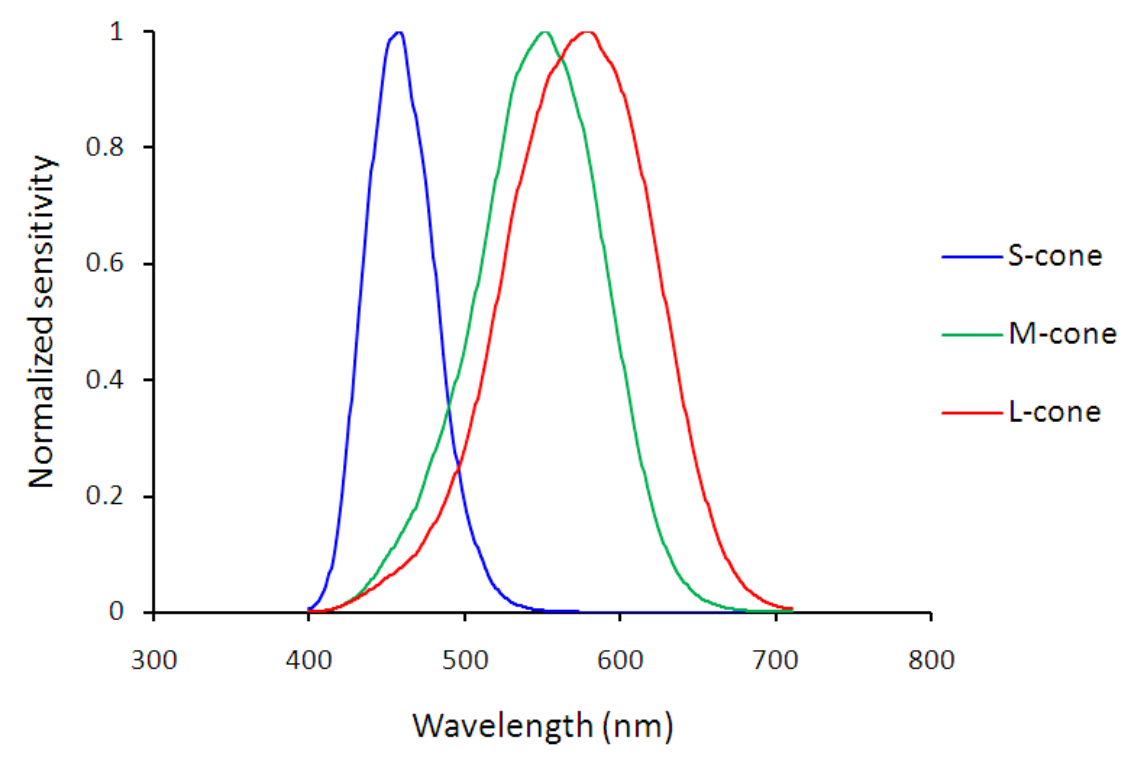

Figure 1.5: Normalized cone spectral sensitivities

Spectral sensitivity curves, measured in vivo, taking into account lens and macula effect on the transmitted light signal based on the Stockman and Sharpe (2000) 10 deg cone fundamentals.

A photoreceptor responds to light by absorbing light quanta (photons) in its photopigment (also called excitation) and by converting this event to an electrical potential difference. Although the probability that a photon is absorbed by a photoreceptor varies by many orders of magnitude with wavelength, its effect, once it is absorbed, is independent of wavelength. This property is called univariance. A photoreceptor is essentially a sophisticated photon counter, the output of which varies according to the number of photons it absorbs. Since a change in photon count could result from a change in wavelength, from a change in intensity, or from both, individual photoreceptor is color 
blind. The visual system is able to distinguish color from intensity changes only by comparing the outputs of two or three cone types (L-, M-, and S-) with different spectral sensitivities. This comparison is performed in the second stage constituted by the retinal ganglion cells and the LGN neurons.

\section{Spectral opponency in retinal ganglion cells}

At the ganglion cell level, cone signals are added and subtracted to provide cell systems, which form the basis of the luminance and chromatic (red-green and blue-yellow) parallel channels of psychophysics. The main classes of ganglion cells, which project in parallel to the LGN are described below (see Lee (2004) for review):

Midget ganglion cells or PC cells: These cells which project to the parvocellular (PC) layers of the LGN, are thought to form the basis of red-green color vision (red-green coloropponent channel) in primates. Midget cells difference of the signals from L- and M-cones (L-M).

Parasol ganglion cells or MC cells: The MC cells which project to the magnocellular (MC) layers of the LGN, are thought form the substrates for luminance vision (luminance channel) in primates. $M C$ cells sum their input from the $L$ and $M(L+M)$ cones and are very selective in changes to luminance.

Small-bistratified cells or KC cells: The KC cells, which project to the koniocellular (KC) layers of the LGN, are thought form the substrates for blue-yellow vision (blue-yellow coloropponent channel) in primates. These cells receive input from the S-cones and compare it to the summed input from the $L-$ and $M$-cones $(S-(L+M))$. A further cell type receives the opposite cone input ((L+M)-S).

Figure 1.6 illustrates the transformation from the cone signals into color-opponent signals. These three channels, - which correspond to the 'cardinal directions' of color (DKL) space (Krauskopf et al., 1982), are functionally independent and transmitted in anatomically distinct retino-geniculo-cortical pathways (Gegenfurtner, 2003). 

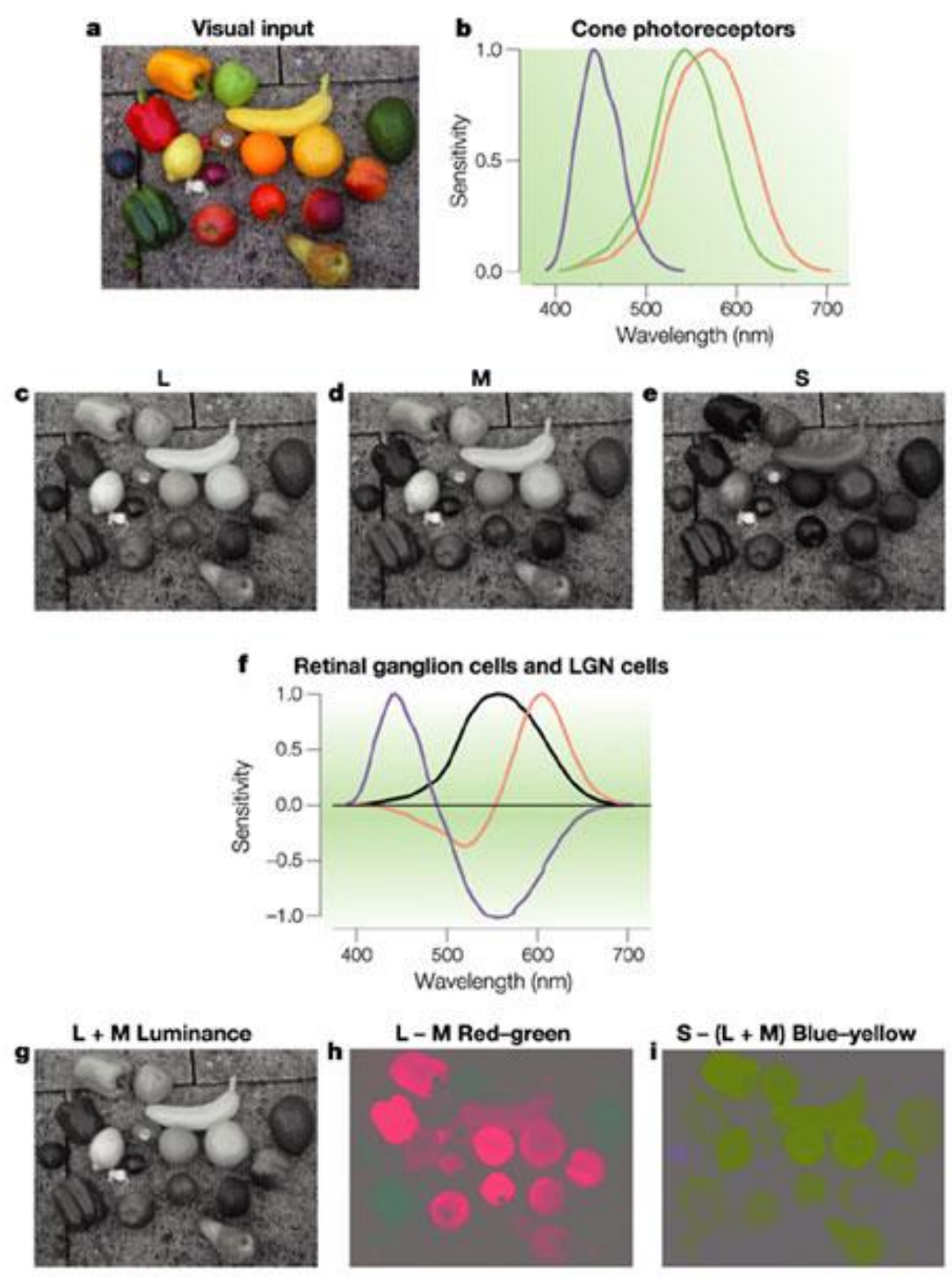

Nature Reviews | Neuroscience

Figure 1.6: Transformation of cone signals into color-opponent signals

Color vision (for example, of the picture shown in a) starts with the absorption of light by three types of cone photoreceptor (L, M and S) in the eye b). The three black and white pictures (c-e) show how the three cone types are excited by the image in $\mathrm{a}$. The L- and $\mathrm{M}$-cone images are similar. The electrical signals generated by these photoreceptors go through complex circuitry f) that transforms the signals into three channels - one carrying luminance and the other two being color-opponent, red-green and blue-yellow (g-i). These coloropponent signals are sent to the visual cortex by way of the thalamic lateral geniculate nucleus (LGN). Source: Gegenfurtner (2003). 


\section{Segregation and spectral opponency in LGN cells}

In the LGN, the segregation of the information from the retina is maintained. Axons of both the spectrally opponent midget retinal ganglion cells synapse on cells of the parvocellular (PC) layers, while the koniocellular (KC) layers appear to receive projections from the smallbistratified cells (Hendry \& Reid, 2000). Axons of the spectrally non-opponent parasol ganglion cells synapse on cells of the magnocellular (MC) layers (Leventhal et al., 1981; Rodieck et al., 1985). Reflecting the qualities of their retinal inputs, the magnocellular cells are very sensitive to luminance and much less so to chromatic contrast, unlike the parvocellular neurons which preferentially respond to chromatic contrast but are responsive to luminance as well (Shapley, 1990). Over $90 \%$ of parvocellular neurons are particularly sensitive to differences in wavelength (DeValois et al., 1966; Derrington et al., 1984).

Like the spectrally sensitive retinal ganglion cells, chromatically sensitive cells of the macaque LGN were shown by De Valois and others, to have opponent responses for different wavelengths (DeValois et al., 1966; DeValois \& Jacobs, 1968). They could be excited by some wavelengths and inhibited by others, in specific combinations. In a major advance, Derrington, Krauskopf, and Lennie (1984) used modulation in the DKL space (named after Derrington, Krauskopf, and Lennie) to study the chromatic properties of cells in the LGN. They showed that the chromatically opponent parvocellular cells specifically responsive to $|\mathrm{L}-\mathrm{M}|$ modulation were much more numerous as those specifically responsive to S-cone modulation. Cells in the magnocellular layers preferred primarily modulation in luminance. They further demonstrated that LGN cells are similar to retinal ganglion cells in that they linearly combine their inputs. Indeed, in PC opponent cells, L-and M-cone signals are almost always opponent; the inputs are received in opposite polarities $(+L-M$, or $-L+M)$. Blue-yellow opponent cells signal the input from the S-cones minus the sum of the L- and Mcones $(S-(L+M))$, or vice versa. 


\subsubsection{Cortical stages of processing}

\section{Functional organization of the visual cortex}

The functional organization of the visual cortex is partially determined by the segregation of inputs from the LGN to area V1. This area is also called striate cortex because of the distinctive white stripe (the stripe of Gennari) created by the myelinated fibres that enter layer 4 of the six cell layers of cortex (see Figure 1.1). Layer 4 is a large layer within area V1 and has distinct subdivisions as illustrated earlier in Figure 1.1. Parvocellular (PC) axons from the LGN, project to the $4 C \beta$ and to $4 A$. They also have a minor projection to layer 1 and the upper region of layer 6 . Layer $4 C \beta$ has output projections to the lower part of layer 3 and from there to layer 2 and the upper sublaminae of 3. Magnocellular (MC) axons from the LGN project to layer $4 \mathrm{C} \alpha$ and have a minor projection to the lower region of layer 6 . From layer $4 \mathrm{C} \alpha$ there is a projection to layer 4B. The main outputs of this layer are to the second (V2) and third (V3) visual areas and then to the medial temporal (MT) area, which appears to be specialized for processing motion signals. Beyond layer 4, most of the MC and PC circuits interconnect different striate layers and then different cortical regions, although the MC and PC streams are less strictly segregated than at the level of the LGN.

All cells contain mitochondria which can be stained by the mitochondrial enzyme, cytochrome oxidase (CO). Higher $\mathrm{CO}$ staining is associated with cells having higher metabolic activity. Throughout V1, there are regularly-spaced regions that stain densely for CO; they appear as slightly irregular ovoids, about 150 × $200 \mu \mathrm{m}$, called blobs (Livingstone \& Hubel, 1984). They are most apparent in cortical layers 2 and 3, but are aligned with fainter CO regions in layers 4 and 6, as illustrated by Figure 1.1. The koniocellular (KC) layers of LGN project directly to layer 3 blobs (Fitzpatrick et al., 1983). There are also intracortical connections to the blobs that provide inputs from both $\mathrm{MC}$ and $\mathrm{PC}$ pathways. It has been suggested that blob cells may be specialized for the analysis of color. The output of striate cortex is primarily from the upper layers $(2,3,4 A$ and $4 B$ ) to extrastriate cortex, most prominently to cortical area V2. Outputs from the lower layers go to deep structures; layer 5 projects to the superior colliculus, while layer 6 projects back to the LGN. 


\section{Chromatic properties of $\mathrm{V} 1$ receptive fields}

Early studies described the existence of color selective cells of the macaque monkey primary visual cortex (Hubel \& Wiesel, 1968; Dow \& Gouras, 1973; Gouras, 1974; Thorell et al., 1984). A turning point regarding the understanding of cortical color mechanisms commenced by the work of Livingstone and Hubel (1984). They reported conglomerates of non-oriented, monocular, color-selective cells in CO-blobs of macaque V1. Within the COblobs, however, an additional color cell type was identified. Livingstone and Hubel had described double-opponent cells in V1, which were first found in the goldfish retina (Daw, 1968). These cells respond most vigorously when their receptive field centers are stimulated with one color and their surround with the opponent color (Daw, 1968; Hubel \& Wiesel, 1968; Conway, 2001). The existence of these cells remains controversial.

Ts'o and Gilbert observed blue-yellow or red-green opponent-color mechanisms in the blobs of V1, and specified additional cells with receptive fields having either a blue-yellow or red-green opponent color mechanism solely in the center, while a broadband inhibitory surround was found (Ts'o \& Gilbert, 1988). They called this additional cell class modified Type II-cells. In addition, they described cells that were color selective and orientation sensitive. These cells were most frequently found between blobs and interblobs regions. In the same study they suggested that CO-blob color cells are organized in clusters with similar sensitivity to opponent colors. Some CO-blobs are described as responding to either redgreen or blue-yellow opponent colors. This suggestion was not supported by later work, which showed that, as opposed to LGN neurons, V1 contains cells that are not exclusively sensitive along the two cardinal color mechanisms (S- $(L+M), L-M)$, but also respond vigorously to mixed colors (Lennie et al., 1990; Solomon \& Lennie, 2005; Conway \& Livingstone, 2006). This mixed color selectivity is also prevalent in macaque V2 (Gegenfurtner et al., 1996) and V3 (Gegenfurtner et al., 1997). 


\section{Chromatic properties of V2 and V3 receptive fields}

One view of striate cortical function is that it segregates information about color, form, motion and depth and distributes that information to extrastriate regions for specialized processing. The CO staining patterns have been thought to reveal parallel pathways that originate from retinal $M C$ and $P C$ streams, forming the inputs to posterior parietal cortex through area MT and to inferior temporal cortex through area V4. Their streams are shown schematically in Figure 1.7. It has been suggested that the temporal stream is specialized for identification of objects based on form and color, while the parietal cortical path is specialized for motion perception, spatial localization and control of attention. Ungerleider and Mishkin (Ungerleider \& Mishkin, 1982) called the temporal and parietal streams the "what" vs. "where" paths, respectively. 


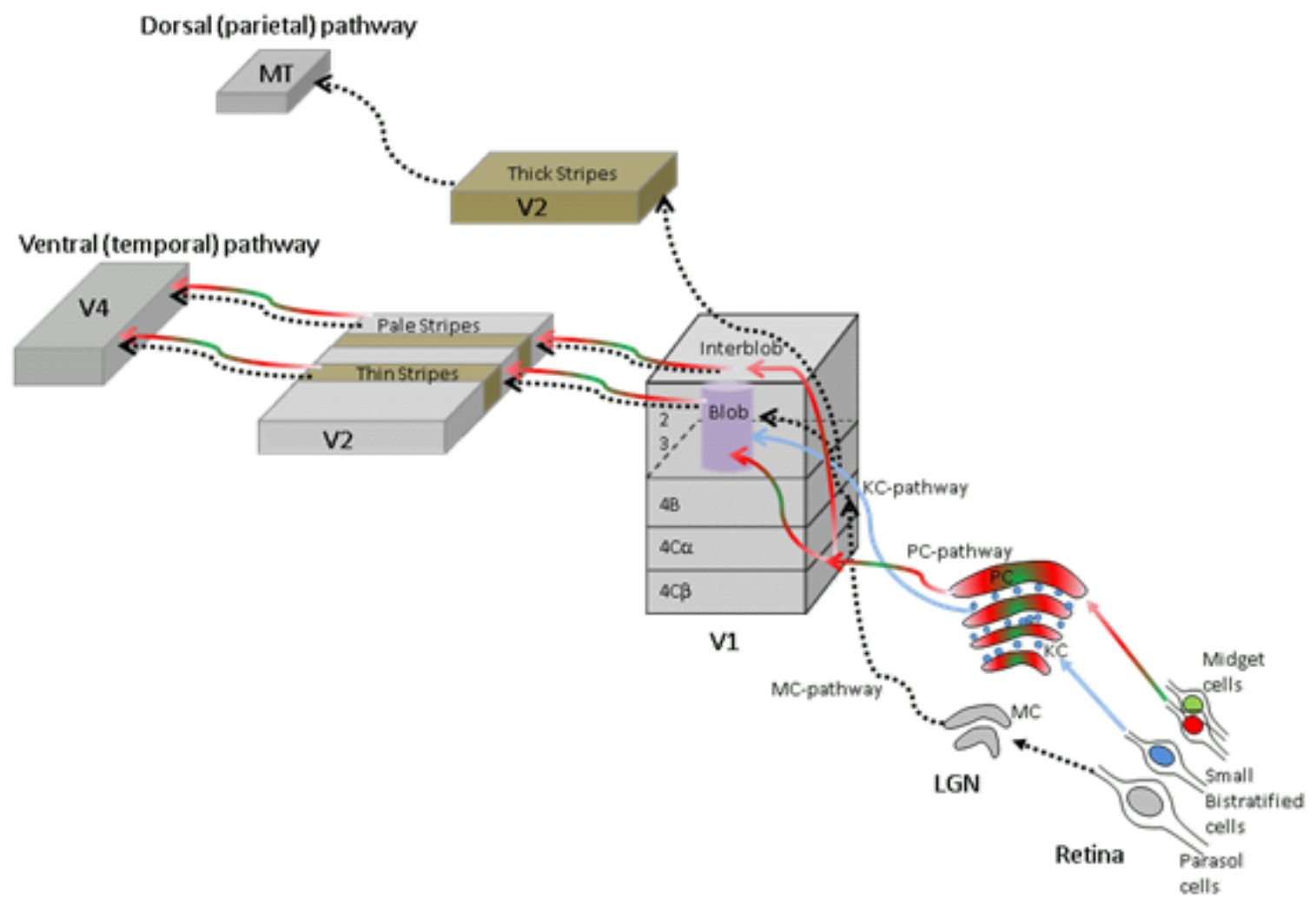

Figure 1.7: A schematic of the major feedforward connections that give rise to serial, hierarchical processing from the LGN through extrastriate cortex.

This schematic shows the parallel pathways through cortical areas V1 and V2 that have been identified by cytochrome oxidase (CO) staining. V3/VP connections between V2 and MT and V4 are not shown here because $\mathrm{CO}$ staining has not revealed divisions there. Magnocellular (MC) pathways are shown by dashed lines, parvocellular (PC) pathways by solid lines (red-green), and a koniocellular (KC) pathway by a blue line. It is not known whether there is a parallel KC path beyond the blobs that is separate from the PC paths, nor is it clear how $M C$ inputs reach the interblobs, although there are numerous lateral connections within cortex. Source: Modified after Kandel et al. (2000).

\section{Area V2}

Like V1, V2 is organized retinotopically, and contains anatomical subdivisions revealed by patterns of CO staining. These CO staining patterns suggest three anatomical divisions or "compartments" in V2: densely stained thin and thick stripes separated by pale stripes (Figure 1.7). These stripes are visible in horizontal sections throughout all V2 layers (Tootell et al., 1988), but the difference between thin and thick stripes is often subtle. The thick stripes can be identified more clearly by counterstaining with the monoclonal antibody Cat- 
301 (DeYoe \& Van Essen, 1985a). The thin stripes receive much of their input from blobs in the upper layers of area V1, while the pale stripes seem to receive their main inputs from PC cells of the interblobs. MC cell signals are relayed from layer $4 \mathrm{~B}$ in V1 to the thick stripes in V2. Livingstone and Hubel (1984a) initially suggested that MC paths are linked to thick stripes, orientation-selective PC paths to thin stripes, and color-coding PC-paths to pale stripes. However, intracortical connections to V1 blobs come from both MC and PC pathways, as noted in the previous section, and it seems unlikely that $M C$ and $P C$ segregation could be more specific than when the signals arrive in area V2. The connections shown in Figure 1.7 reflect this mixed input, but do not rule out the possibility of some bias in strength of inputs along the lines suggested by Livingstone and Hubel. In one of the few quantitative studies of V2 receptive fields, Levitt et al. (1994) studied chromatic and achromatic receptive field properties using diffuse stimuli and gratings adjusted to the optimal spatial frequency for each cell. The results were quite similar to those obtained for striate cortex. Most V2 cells were more responsive to luminance modulation than chromatic modulation. Cells that were highly color-selective tended to have poorer orientation selectivity, although some cells tuned to orientation also responded to chromatic modulation. Consistent with results of Cottaris and DeValois (1998) for V1, Levitt et al. (1994) reported that many V2 cells combined cone signals nonlinearly. Cells that were selective in their response to color, size and motion were found mainly, but not entirely, in different V2 stripes. Most color-selective cells were found in the upper layers of V2. Within the various layers, there were more color-selective cells in the thin and pale stripes, consistent with greater PC than MC input. Cells more responsive to chromatic than luminance modulation were found only in the thin stripes. Gegenfurtner et al. (1996) reached similar conclusions after mapping V2 receptive fields with probes for color, motion and form. However, many cells were selective to more than one of these types of stimuli, regardless of their CO compartment. Kiper et al. (1997) concluded that one of the major differences between V1 and V2 is in the higher proportion of cells in V2 ( 35\%) with narrow color tuning, a property that some researchers suggested to be characteristic of cells in area V4. They found very few cells with double-opponent receptive fields. 


\section{Area V3/VP}

Cortical area V3 receives input from layer 4B (dominated by MC cells) of striate cortex as well as from area V2. This cortical area does not have regions that stain selectively for $\mathrm{CO}$, but it is known to have inputs from both $M C$ and $P C$ pathways. The major efferent projections from V3 are to areas MT and V4. Most of the cells in V3 respond selectively to orientation, direction of motion, or binocular disparity. While earlier studies suggested that cells in area V3 have little or no selectivity to color, several more recent studies reach different conclusions (Felleman \& Essen, 1987). Gegenfurtner et al. (1997) using the same methods described previously for their studies of area V2, verified that directional selectivity was an important characteristic of cells in V3. These and other data support the conjecture that this area is important in processing higher-level motion signals. In addition, they found about half of V3 cells to be color selective, essentially the same proportion previously found in area V2 using the same criteria. Many of these cells had more S-cone input than these researchers observed in cells of area V2, but the input was weak and combined with strong additive signals from $\mathrm{M}$ - and L- cones. Chromatic contrast sensitivity was generally higher for V3 than V2 cells. Finally, some of the directionally-selective cells responded to isoluminant chromatic gratings, leading Gegenfurtner et al. (1997) to conclude that there is significant interaction between color and motion signals in this extrastriate area.

\section{Cortical area V4}

The topographic mapping of visual space is generally less strict at those higher cortical levels where specialized processing has been suggested to occur. The existence of specialized cortical regions is also reasonable from computational considerations; breaking a task into components, is an efficient way to cope with complex tasks. Such specialization within cortex would also minimize the number and length of connections needed to link neural representations of common properties across widely separated portions of a stimulus (Barlow, 1986). However, this specialization might only be advantageous after earlier stages have accomplished preparatory steps common to all specialized modules; otherwise, there would be unnecessary duplication of circuitry. Zeki suggested that area V4 is a cortical module specialized for processing of color (Zeki, 1983a) and area MT for motion. This role of 
MT has been largely sustained; the hypothesis about V4 has been less completely supported (Schein \& Desimone, 1990). Zeki's hypothesis has nevertheless engendered a great deal of research regarding the properties of cells in these areas, their anatomical connections, and the consequences of circumscribed cortical damage.

Area V4 has a topographical organization, but the mapping is considerably more complicated than that found in V1. The topography is difficult to discern because it is in a highly convoluted region of cortex and the receptive fields are large. While the representation in V1 is concerned with the contralateral visual field, V4 is concerned primarily with only about $30 \mathrm{deg}$, mostly of the central visual field. Part of this field ( $5 \mathrm{deg}$ ) is ipsilateral, indicating that it has major inputs via the corpus callosum from the opposite hemisphere, presumably from the corresponding area V4. The main inputs to area V4 are from the thin and pale stripes of V2 (DeYoe \& Van Essen, 1985b) and from V3/VP. The latter input may be one of the sources of the MC-cell signals that it receives. These MC-cell inputs are apparently not appreciably segregated from those from PC-cell dominated paths (Ferrera et al., 1994). V4 provides a major source of input to inferotemporal cortex, which is known to be essential for visual recognition of objects. It is generally agreed that a high percentage of cells in area V4 respond selectively to color, although the exact proportion clearly depends on the criteria used. The spectral bandwidth of V4 cells is similar to that found in LGN PC-cells using similar methods, suggesting that V4 responses are based on color opponency established at a prior level, but half-wave rectified in the cortex (Demonasterio \& Schein, 1982). Receptive fields of area V4 are 4-6 times as large as those at comparable eccentricity in V1, and an individual receptive field may receive input from thousands of ganglion cells (Schein \& Desimone, 1990). Despite their large receptive fields, some V4 cells appear to be as selective as cells in striate cortex in their tuning for size, spatial frequency, and orientation (Desimone et al., 1985; Desimone \& Schein, 1987). Finding the optimal stimulus for a V4 cell can be difficult due to the complexity of their receptive fields (Werner). 


\subsection{Fundamentals of $\mathrm{fMRI}$}

\subsubsection{Basic principles of magnetic resonance imaging (MRI)}

Magnetic resonance imaging (MRI) is an imaging technique most commonly used in medical settings to obtain high-resolution images of the brain and other structures of the human body. MRI, in principle, exploits the magnetic properties of atomic nuclei, therefore it is also known as nuclear magnetic resonance imaging, or NMRI. Even though NMRI does not involve any radioactivity, the term 'nuclear' has nonetheless been omitted in medical circle to avoid patient concern, and the familiar appellation 'MRI' has been adopted. Functional magnetic resonance imaging ( $\mathrm{fMRI}$ ) is essentially an adaptation of the standard MRI, and is one of the most recently developed forms of neuroimaging. In the following sections some basic issues concerning the physical and physiological principles of fMRI will discussed.

At the core of the technique is a large permanent magnetic field $\left(B_{0} ; 2.9\right.$ Tesla in the current experiments). Protons that are placed into the field align their spins either parallel or antiparallel to $B_{0}$. The parallel state is energetically favorable compared to the antiparallel state. In other words, energy is required to move a proton from a state where its spin is parallel to $B_{0}$ into the antiparallel state. Consequently, if a proton falls from the antiparallel to the parallel state, energy is released. Due to thermal motion the total difference between protons in parallel and antiparallel states is very small, there are, however, an enormous number of protons in a tiny volume of tissue and it therefore becomes possible to exploit the effect of $B_{0}$ on them. In order to obtain a signal, we briefly apply an electromagnetic pulse at a specific frequency (Larmor Frequency). This pulse provides sufficient energy to move a tiny portion of protons from the parallel to the antiparallel spin state. The macroscopic manifestation is a flipping of the magnetization vector $(z)$ by a certain flip angle into the transverse plane. When the excitation pulse is switched off, protons with antiparallel spins gradually return to the parallel spin orientation. Macroscopically, this leads to a cycling of the $z$ vector in the transverse plane (with the characteristic frequency) gradually approaching its original configuration in the longitudinal plane. This process is characterized by two relaxation times, the longitudinal relaxation time, $T_{1}$ and the transverse relaxation time, $T_{2}$. During the relaxation process, the cycling magnetization 
vector induces a current in the receiving coil which is recorded and then converted into an intensity signal. The signal decays rapidly with time depending, in theory, on the rate of transverse relaxation, given by $1 / T_{2}$. In practice, however, signal decay occurs faster than would be expected from a $T_{2}$ - dependent decay. The reason for this can be seen when considering that the relaxation frequency is directly proportional to the strength of the magnetic field.

$$
\omega=\gamma B_{0}
$$

In the above equation $\omega$ represents the relaxation frequency and $v$ stands for the gyromagnetic moment (which is $42 \mathrm{MHz}^{*} \mathrm{~T}^{1}$ for protons). It follows from equation (1.5.1) that small but inevitable fluctuations in a magnetic field that is not perfectly homogeneous will lead to slightly different relaxation rates at different points in the tissue. This "dephasing" accumulates over time and leads to a progressive decrease in the signal. In order to take into account these effects, an effective transverse relaxation time $\left(T_{2}{ }^{*}\right)$ is usually stated. Thus, the amount of detectable signal is a function of the effective transverse relaxation time, $T_{2}{ }^{*}$.

Spatial encoding in MRI is made possible by applying additional magnetic fields during the acquisition (and excitation) period using gradient coils. These gradients bring about systematic changes in the magnetic field which lead to excitation and emission variations. Analyzing and transforming these variations eventually yields spatially localizable information. As an example, consider the problem of slice selection. In this case, a magnetic field gradient is applied during the excitation pulse such that, for example, the anterior end of the body experiences a stronger $B_{0}$ field than the posterior end. As the Lamor frequency depends on the magnitude of $B_{0}$, giving an excitation pulse with a very precisely determined wavelength will only excite the protons in the part of the body which meets the resonance condition (equation (1.5.1)). Consequently, a signal will only be recorded from the selected slice. 


\subsubsection{Blood oxygenation level dependent (BOLD) fMRI}

Before the advent of human fMRI, animal experiments had shown that the use of exogenous contrast agents (e.g. Gadolinium) can alter the signal strength during magnetic resonance imaging. Subsequent research (Ogawa et al., 1992) established that an endogenous contrast agent, deoxygenated hemoglobin $(\mathrm{rHb})$, could be used to monitor brain activity. When oxygenated hemoglobin releases its bound oxygen into the tissue, it becomes a paramagnetic substance which causes local dephasing of the relaxation process leading ultimately to a loss of the water proton signal, as discussed above. In other words, if MRI sequences are chosen that are susceptible to changes in $T_{2}{ }^{*}$ (gradient echo sequences with a long echo time, TE) the amount of signal obtained will be inversely proportional to the concentration of $r H b$. This technique, known as "blood oxygen level dependent functional magnetic resonance imaging" (BOLD fMRI), can be used for functional investigations of the brain because neural activity and blood oxygenation level are tightly coupled. Unfortunately, this relationship is non-trivial as multiple parameters with an influence on blood oxygen level change in response to a change in neural activity. Most importantly, however, an increase in neural activity is accompanied by a swift increase in the rate of cerebral blood flow (CBF) by $40 \%$, resulting in blood hyperoxygenation (reduced concentration of $r H b$ ). This effect leads to an fMRI signal gain, which is known as the BOLD response. The BOLD response has been extensively characterized (Fransson et al., 1998). In response to a brief visual stimulus, the signal increase can be detected 1.5 to 2 seconds after stimulus onset (due to hemodynamic latency) and achieves a maximum of around $4 \%$, with respect to the previous baseline, by 5 to 7 seconds after stimulus onset. 


\subsection{Thesis overview}

The first aim of the experiments is to investigate how the human visual cortex responds to chromatic modulation in the foveal compared to the peripheral parts of the visual field. The second aim is to investigate the temporal frequency dependent specialization of the cortical visual areas to chromatic modulation. The intention is to provide neurophysiological evidence relevant to the behavioral loss of sensitivity to discriminate colors in peripheral vision as well as to the loss of sensitivity to detect fast chromatic modulation. A detailed description of the research aims is provided in the following sections.

\subsubsection{Color discrimination deteriorates across the visual field}

Visual abilities change over the visual field. Humans possess a very high visual acuity and contrast sensitivity in foveal vision (humans can resolve spatial frequencies up to $40 \mathrm{cpd}$ in achromatic vision), whereas performance deteriorates rapidly with increasing visual field eccentricity (Virsu \& Rovamo, 1979). Studies of peripheral vision, however, are susceptible to stimulus parameters. Specifically, stimulus size and spatial frequency are critical factors, and must be taken into account by scaling in terms of 'cortical magnification' - the fact that progressively less neural resource is assigned to more peripheral regions of visual space. Often, when stimulus size is suitably increased (and spatial frequency decreased) for peripheral stimuli, foveal and peripheral visual performance can be made equivalent (Daniels \& Whitteridge, 1961; Cowey \& Rolls, 1974; Rovamo et al., 1978; Virsu \& Rovamo, 1979; Virsu et al., 1987). The above is valid for studies using achromatic (luminance modulated) stimuli, and is usually referred to as M-scaling. Color discrimination in peripheral vision was classically considered to be poor, but some studies have shown the critical importance of size of the stimulus in color naming in peripheral compared to foveal vision; with large enough stimuli, color naming in peripheral vision is as in the fovea (Abramov \& Gordon, 1977; Noorlander et al., 1983; Abramov et al., 1991; Vakrou et al., 2005; Hansen et al., 2009). However, chromatic discrimination is worse in peripheral compared to central vision even after M-scaling, especially for red-green sensitivity, which declines steeply away 
from the fovea (Anderson et al., 1991; Mullen, 1991; Stromeyer et al., 1992; Mullen \& Kingdom, 1996; Mullen \& Kingdom, 2002; Mullen et al., 2005).

As described in an earlier section of this chapter, in primates, ganglion cells of the midget, parvocellular pathway are thought to be the origin of the red-green channel for color vision. Two possibilities have been proposed for the red-green chromatic sensitivity in foveal and peripheral vision, respectively (Vakrou et al., 2005). In fovea, chromatic opponency results from the fact that a midget ganglion cell's center is driven by a single cone (e.g. L-cone) via a bipolar cell, while the surround receives input either from a different cone type (e.g. M-cone) (selective surround) or from mixed cone-types (mixed surrounds). Both schemes result in color opponency (Figure 1.8, top) due to the fact that the receptive field center receives its input from a single cone type via a single bipolar cell - the so called 'private-line arrangement' (Wiesel \& Hubel, 1966; Wässle \& Boycott, 1991; Reid \& Shapley, 1992; Calkins et al., 1994; Reid \& Shapley, 2002). The discussion as to whether surrounds receive selective or mixed cone input, or are partially selective, has not been resolved (Lee, 2008). However, with increasing eccentricity, midget ganglion cells receive input from large numbers of bipolar cells and the single-cone input to the receptive field center is lost (Figure 1.8, bottom). According to the 'random wiring' hypothesis (Lennie et al., 1991; Dacey, 1993), the qualitative loss of red-green chromatic discrimination in peripheral vision has been associated with random wiring causing a loss of chromatic responsivity in peripheral midget ganglion cells (Mullen, 1991; Mullen \& Kingdom, 1996; Mullen \& Kingdom, 2002; Newton \& Eskew, 2003). 


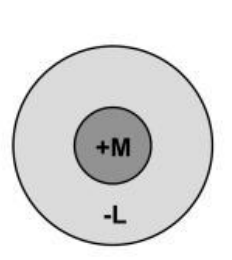

cone-selective hypothesis parafovea

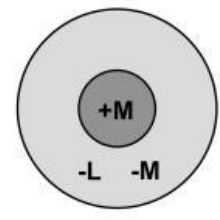

random-wiring hypothesis

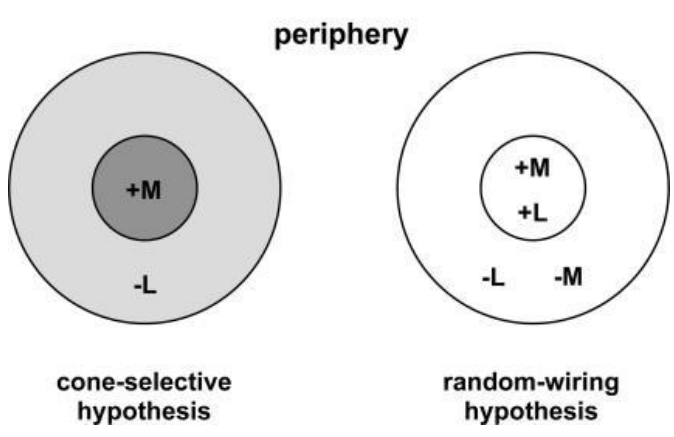

Figure 1.8: Models proposed for L-M chromatic opponency, based on center-surround receptive field antagonism

The resulting opponency is determined by the relative weights of the cone input to the center versus the surround. In the parafovea a 'private-line' arrangement exists, where a single cone type (+M here) provides input to the center of the ganglion cell's receptive field, whilst the surround gets input from another cone type ('cone-selective' hypothesis) or from mixed cone types ('random-wiring' hypothesis). Chromatic opponency is preserved in both cases. In the peripheral retina midget ganglion cells' receptive fields are much larger and receive convergent input from a number of photoreceptors. The cone-selective hypothesis postulates a selective circuitry where both center and surround receive input from a single cone type; chromatic opponency is preserved. The 'random-wiring' hypothesis postulates mixed input both to the center and surround of receptive field, resulting in a non-opponent peripheral cell. Source: Vakrou et al. (2005).

\section{Evidence against 'random-wiring' hypothesis}

However, recently it has been shown that cone-specific connectivity is likely to exist in the peripheral parts of the visual field. Martin et al. (2001) presented red-green chromatic modulation at eccentricities from 20 to $50 \mathrm{deg}$ and recorded midget ganglion cell responses from macaque monkey's retina. Both peripheral and foveal ganglion cells showed almost equivalent chromatic responsivity. When the same stimuli were shown to a human subject, they observed that the psychophysical performance deteriorates rapidly with eccentricity (Figure 1.9). They argued that the psychophysically demonstrated deterioration of color discrimination in the peripheral visual field occurred at a cortical site; Derrington et al. (2001), however, suggested that information from the cone opponent cells is not used 
during further cortical processing. It should also be noted that the psychophysical deterioration of red-green sensitivity is already marked at 10 deg eccentricity, at this eccentricity the 'private line' wiring is still maintained; substantial convergence from midget bipolar cells onto midget ganglion cells only occurs at eccentricities of 20 deg or more.

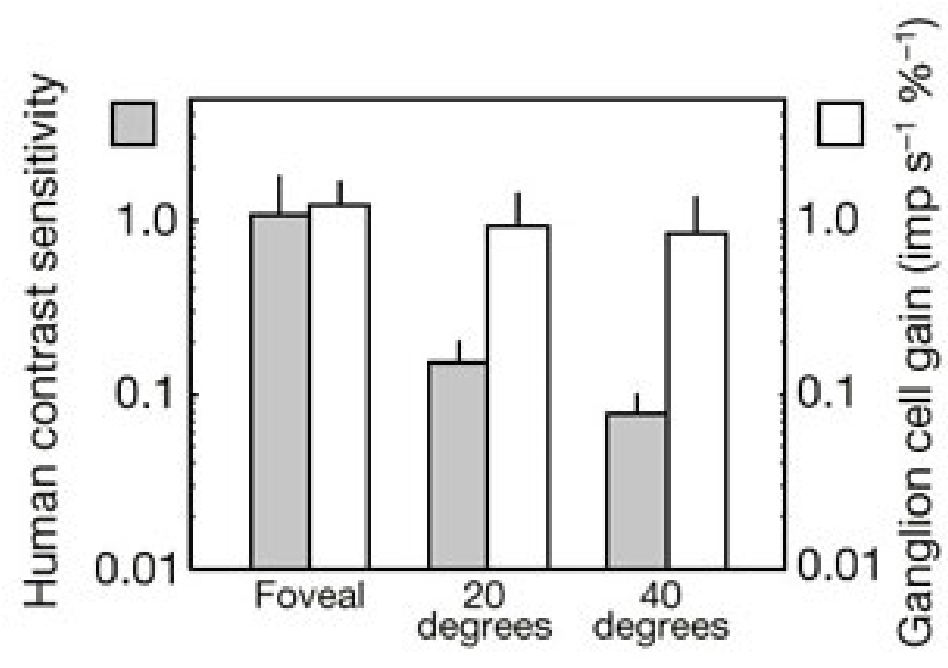

Figure 1.9: Comparison of psychophysical sensitivity of three human observers with opponent PC cell contrast gain to isoluminant red-green modulation Source: Martin et al. (2001).

\subsubsection{Eccentricity-dependent chromatic responses in human visual cortex}

Our understanding of cortical specialization for peripheral color vision in humans is very limited and restricted mainly to the primary visual cortex (V1). To our knowledge, until now, only two studies (Vanni et al., 2006; Mullen et al., 2007) have used fMRI to investigate the distribution of eccentricity-dependent chromatic response in V1. Both studies suggest a decline in red-green response across eccentricity, and in contrast, a shallow or no decline in luminance and blue-yellow responses. Further, the authors were inclined to suggest that their physiological findings match closely to the previously observed psychophysical loss of red-green chromatic sensitivity in peripheral vision (Mullen, 1991; Mullen \& Kingdom, 2002; Mullen et al., 2005). However, careful inspection of the fMRI data from Mullen et al. (2007) reveals that there is a substantial inter-subject variability in the response pattern, with some 
subjects showing no or little decline in red-green responsivity across eccentricity. Moreover, they show eccentricity-dependent responses only for stimuli matched in multiples of detection threshold (MDT). The stimuli (red-green, luminance, and blue-yellow) matched in MDT appeared to have approximately the same visibility, however, the cone-contrast varied between different stimuli as was the case in Vanni et al. (2006). It has been shown, that better comparison of $\mathrm{fMRI}$ responses is possible in early visual areas only if stimuli are matched in terms of their cone-contrast values (Kleinschmidt et al., 1996; Engel et al., 1997a; Liu \& Wandell, 2005).

The experiments conducted by Vanni et al. (2006) constitute a more extensive study. This study employed three different spatial patterns. In their first experiment, with checkerboard stimulus patches (M-scaled), an even distribution of red-green response was observed across eccentricity. For stimuli (second experiment) with a constant checkerboard pattern across eccentricity a decline in red-green response was seen, and this is likely to be because constant check size (without M-scaling), create a non-optimum spatial frequency profile across eccentricity, resulting in a response decrease. Finally, in their third experiment, spatially Gaussian modulated white-noise patterns (spatial frequency was restricted to a 0.2-0.8 $\mathrm{cpd}$ frequency band) were used, for which an eccentricity-related decline in red-green response was reported. Once again, the response drop may have been due to the lack of scaling of spatial frequency in the stimulus pattern. However, strikingly, no decline in blue-yellow and luminance responses was observed across eccentricity, which might imply that these three pathways have different spatial frequency profiles across eccentricity. It has been shown in psychophysical experiments (Vakrou et al., 2005), that red-green (Hansen et al., 2009), luminance, and blue-yellow contrast sensitivities can be matched across the visual field by accounting for changes in scale of the underlying mechanism, i.e. different scaling of spatial frequencies applies for red-green, luminance, and blue-yellow stimuli across eccentricity. However, it should be noted that M-scaling is based on retinal cell ganglion cell distribution and cortical magnification across eccentricity, and it is not clear why this should be different for different pathways. It is true that the blueyellow system is phylogenetically ancient in comparison with L-M cone-opponent pathway. On the other hand, the sparse S-cone density in the central retina may also affect the 
psychophysical scaling stimulus size for blue-yellow. All in all there is no consensus as to which (or if any) of the human cortical areas provide neural substrates for the behaviorally observed loss of red-green chromatic sensitivity in peripheral vision.

One further aspect of spatial frequency selectivity that has received little attention is visual resolution of $P C$ and $M C$ cells for chromatic and luminance modulation. Both cell groups show similar visual resolution for luminance gratings (Derrington \& Lennie, 1984; Crook et al., 1988), extending up to $30-40 \mathrm{cpd}$ in the fovea, which is about the resolution limit of the macaque (Cavonius \& Robbins, 1973). It is well established that visual resolution of ganglion cell is determined by the center diameter (Peichl \& Wassle, 1979), with one cycle period at the resolution limit roughly corresponding to the center Gaussian radius. For a PC cell, the resolution limit for luminance and chromatic gratings should thus be similar, since the center size is the same in both cases. On the other hand, visual resolution psychophysically is much less for chromatic ( 10 cpd) than for luminance ( $\sim 0 \mathrm{cpd}$ ) gratings. This is illustrated in Figure 1.10 (Lee B. B. \& Sun H.; unpublished observations), which shows psychophysical sensitivity curves and responses of a typical PC cell for red-green chromatic and luminance modulation. Thus, there is a further mismatch between psychophysics and physiology in this instance; presumably spatial summation at a central site reduces psychophysical sensitivity to high spatial frequency chromatic gratings. 
a

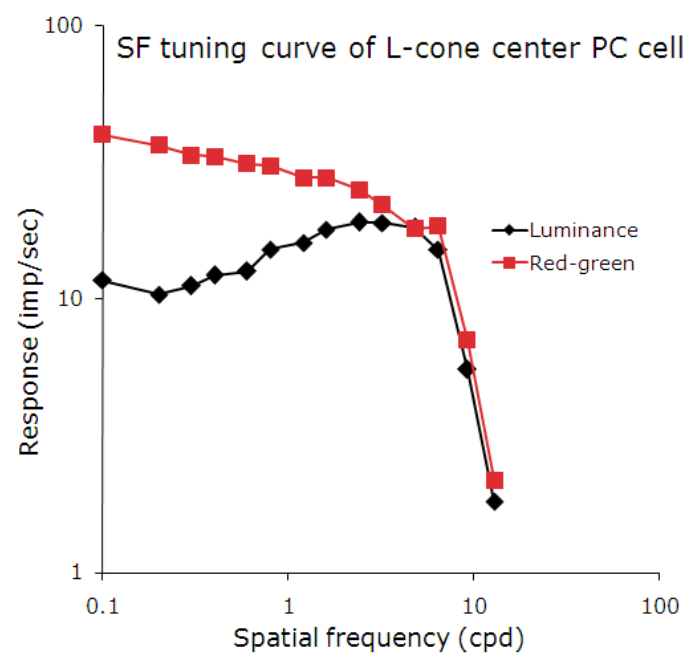

b

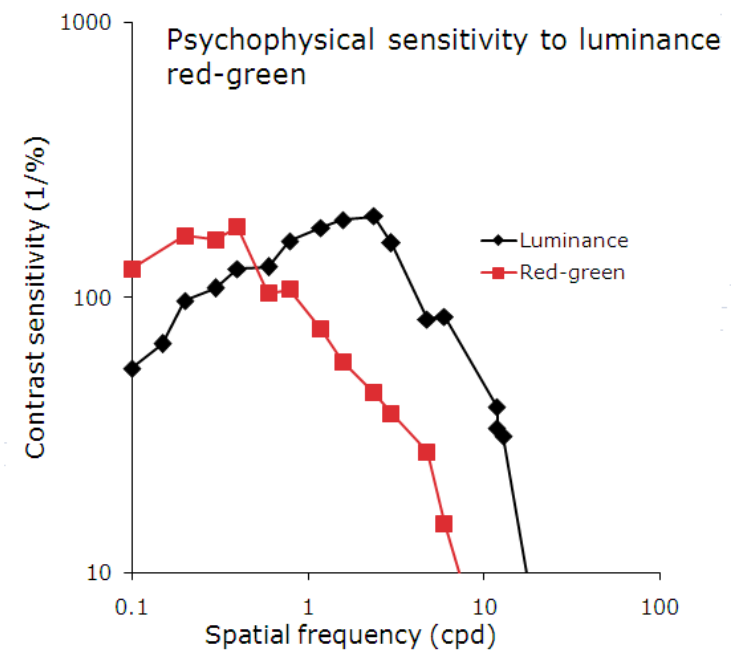

Figure 1.10: Spatial frequency tuning curves: $P C$ cell vs. psychophysics

a) Response of parafoveal +L-M parafoveal midget ganglion cell to luminance and red-green chromatic gratings as a function of spatial frequency. Gratings (30\% RMS cone contrast) were drifted across the receptive field at $4 \mathrm{~Hz}$. The cell responds to both gratings up to a similar spatial frequency limit. This is consistent with the resolution limit of the cell being determined by center size irrespective of grating type. b) Psychophysical thresholds (foveal viewing) for luminance and red-green chromatic gratings as a function of spatial frequency. Visual resolution differs for the two grating types, as described in previous studies (Mullen, 1985). Source: Data are from Lee B. B. and Sun H.; (unpublished observations).

In this thesis, I performed a Spatial Frequency Experiment to study the chromatic and luminance responses as a function of eccentricity to grating stimuli of various spatial frequencies. $\mathrm{fMRI}$ responses were measured in V1 as well as retinotopically mapped visual areas in the ventral visual pathway using stimuli which produced a high cone-contrast. Our results suggest an interaction of the spatial frequency with eccentricity-dependent responses in V1. Furthermore, different $\mathrm{M}$-scaling functions are required to obtain approximately an even distribution of red-green, luminance, and blue-yellow responses across eccentricity. The findings suggest that area V1 may not contribute the psychophysical loss of red-green chromatic discrimination sensitivity. Interestingly, however, activation patterns in area V4 suggested a probable role of the area in the psychophysical sensitivity loss. 


\subsubsection{Psychophysical chromatic contrast sensitivity deteriorates at high temporal modulation frequencies}

If the luminance of a stimulus is modulated in time, sensitivity of the human observer is maximal at approximately at $10 \mathrm{~Hz}$; moreover, humans are capable of detecting luminance changes to modulation frequencies up to $\sim 40-50 \mathrm{~Hz}$ (Swanson et al., 1987). The ganglion cells of the magnocellular (MC) pathway, which are thought to provide a neural substrate for a psychophysical luminance channel show similar temporal frequency tuning characteristics. For chromatic modulation between two lights (such as red-green and blueyellow) matched in luminance, sensitivity of the human observer is maximum at $2 \mathrm{~Hz}$ or less and deteriorates rapidly at modulation frequencies beyond about $5 \mathrm{~Hz}$, and ability to detect chromatic alternation is lost around $12-15 \mathrm{~Hz}$; critical flicker fusion (CFF) occurs. Remarkably, however, the responses, of the ganglion cells of the parvocellular (PC) pathway, which provide neural substrate for our red-green vision, persist up to $30-40 \mathrm{~Hz}$ (Lee et al., 1990). Temporal frequency tuning curves for red-green chromatic modulation are compared in Figure 1.11. This same finding holds for cells with S-cone input (Yeh et al., 1995). This inconsistency between psychophysics and ganglion cell response for red-green and blueyellow vision hints that high temporal frequency chromatic information is subjected to lowpass filtering post-retinally, i.e. in cortical visual areas.

There is considerable evidence for the existence of dorsal and ventral cortical streams in humans and non-human primates alike (Ungerleider \& Mishkin, 1982; Goodale \& Milner, 1992). Whereas the dorsal stream is mainly involved in motion processing, the ventral stream is mainly concerned with object and form vision. Motion processing seems to be dominated by luminance mechanisms (Maunsell et al., 1990), although slower chromatic motion mechanisms may also exist. The ventral stream receives both luminance and chromatic input (Merigan \& Maunsell, 1993b; Merigan et al., 1997). It seems likely that motion processing requires higher temporal resolution than form analysis, and cortical filtering of high temporal frequency information is supposed to be more marked in the ventral than in the dorsal pathway. 


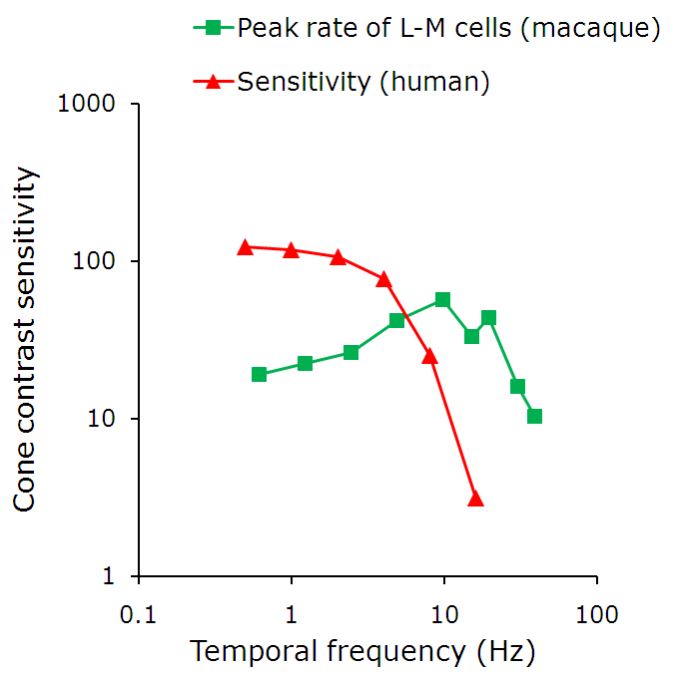

Figure 1.11: Cell and psychophysical sensitivity to chromatic and luminance modulation

Sensitivity has been converted to cone contrast using the cone fundamentals. Source: Adapted from Lee et al. (2007).

\subsubsection{Cortical responses to temporal frequency-dependent chromatic information}

In the past electrophysiological recordings in macaque monkeys have shown that cells of the lateral geniculate nucleus (LGN) and primary visual cortex (V1) respond to high temporal frequency chromatic modulation (Gur \& Snodderly, 1997), at least for red-green modulation. However, little known as to how the extrastriate cortex represents high temporal frequency information.

fMRI studies in humans have attempted to locate a cortical specialization for color and motion (high temporal frequency color information). It has been shown that high temporal frequency red-green and luminance responses are maintained at the entry to the visual cortices, i.e. the primary visual cortex (V1) (Liu \& Wandell, 2005; Jiang et al., 2007). There have been incidental observations suggesting that blue-yellow responses decrease with increasing temporal frequency in V1. Further, there is some evidence suggesting that extrastriate areas have different temporal frequency tuning characteristics, with areas in the ventral pathway preferring low temporal frequencies, while on the other hand dorsal areas responding equally well to both low as well as high frequencies. There is also evidence that 
high temporal frequency information can reach area V4 in humans (Jiang et al., 2007). As for specialization for temporal frequency, Liu \& Wandell (2005) have proposed a common functional network comprising visual areas $\mathrm{V} 3 \mathrm{a}$ and $\mathrm{MT}$, despite these areas being well separated on the cortical surface. The proposed network is believed to be responsible for processing high temporal frequency information; however, the chromatic component may not be encoded efficiently. Recently, the findings of (Grueshow, 2007) suggest a functional color-motion-network comprising areas $\mathrm{V} 3 \mathrm{a}, \mathrm{MT}$, and $\mathrm{V} 4$, which raises some questions because of their widely separated locations; however, the notion, that there is an extensive cross talk between these remotely situated areas cannot be disregarded. Although these previous $\mathrm{fMRI}$ studies have been successful in unraveling, to some extent, how the striate and extrastriate areas handle high temporal chromatic information, more quantitative measurements are necessary to investigate visual areas individually regarding their temporal frequency tuning characteristics.

To ascertain the neural loci, which could possibly contribute to the psychophysical sensitivity loss to high temporal frequency chromatic information, I performed a Temporal Frequency Experiment, in which chromatic and luminance responses were measured as a function of temporal frequency. I measured $\mathrm{FMRI}$ responses in LGN, and other retinotopically mapped cortical visual areas using high cone-contrast stimuli. Our findings indicate that both chromatic and luminance high temporal frequency information reaches V1 through LGN. High-frequency blue-yellow responses were lost in all areas from V1 onward. In MT color and luminance responses increased with temporal frequency, indicating its specialization for high temporal frequency information. Ventral areas showed a drop in response amplitude at high temporal frequencies, V4 showing a greater specialization for color at the lowest temporal frequency used in the experiment. We propose three clusters of visual areas with specialization for different temporal frequencies; one of the clusters could have a potential role in the psychophysical sensitivity loss. 


\section{2}

Materials and Methods 


\subsection{Overview of experiments}

Using a combination of three fMRI experiments, the spatial and temporal characteristics of chromatic and luminance information processing in human retinotopic visual areas are investigated in this thesis. In the Retinotopic Mapping Experiment, the lateral geniculate nucleus (LGN) and cortical visual areas (V1, V2v, V2d, VP, V3d, V3a, V4, and MT) were identified. Further, for each of the latter (except MT), maps representing the visual field were obtained. In the Spatial Frequency Experiment, the aim was to measure the distribution of chromatic and luminance responses in cortical visual areas as a function of visual field eccentricity. In addition, the effect of spatial frequency of the stimulus on the responses was investigated at different eccentricities. Finally, in the Temporal Frequency Experiment, the responsivity across visual areas to modulation of the chromatic and luminance pathways at various temporal frequencies was assessed.

\subsection{Subjects}

Healthy student volunteers ( 3 female and 3 male; mean age $24 \pm 4$ years) from the University of Göttingen participated in the final fMRI experiments as subjects (a total of 25 subjects participated in the preliminary experiments during the course of this thesis work). Six subjects participated in retinotopic mapping and temporal frequency experiments, whereas three out of the six participated in the spatial frequency experiments. No participant had a history of psychiatric or neurological disorder. Informed written consent was obtained from all subjects prior to participation in each experimental session. All subjects received training for maintaining their fixation (fixation task) to the center of the stimulus screen during visual experiments. Trial experiments were conducted in order to familiarize the subjects with the scanning environment. The experimental procedures strictly conformed to the institutional guidelines. All subjects had normal visual acuity and were color-normal trichromats as assessed by the Farnsworth-Munsell 100-Hue Test (Farnsworth, 1943; Rigby et al., 1991). 


\subsection{Color discrimination test}

Briefly, the Farnsworth-Munsell 100-Hue Test (Farnsworth, 1943; Rigby et al., 1991) consists of 84 color caps. These color samples define equal, barely distinguishable hue increments around the hue circle of the Munsell Color Order System (Nickerson, 1940; Tyler \& Hardy, 1940; Nickerso.D, 1969) at a low chroma (the colors appear to be unsaturated). The hue circle is divided into four trays (Figure 2.1), and all colors within each tray have a constant lightness. Thus, only hue can be used to discriminate between the color samples in each tray. Subjects are asked to take all the color caps within one tray at a time and sort them left to right in a continuous hue sequence (for example, from red to yellow or turquoise to violet). On the back of each cap is a number from 1 to 84 indicating its correct place in the hue sequence around the Munsell color circle. When the subject had finished sorting, the caps were turned over and errors in their ordering were scored as the sum of the differences between the cap number and its rank in the sorted sequence. When this task is given to subjects with normal color vision, few score (Figure 2.2) perfectly, and most confuse the ordering of some hues, but the errors are distributed randomly around the hue circle and involve hues next to one another in the sequence. When individuals with congenital color deficiencies take the test, they tend to confuse widely different hues. Moreover, the confusions fall on specific areas of the hue circle.

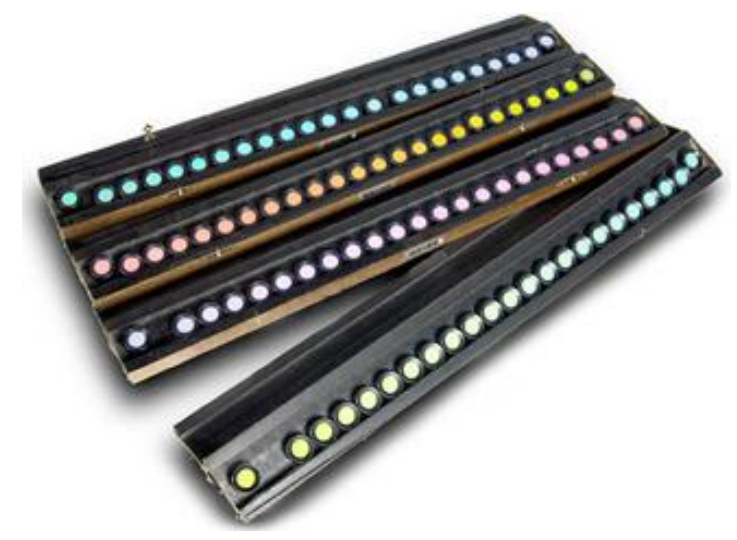

Figure 2.1: Farnsworth-Munsell 100-Hue Test trays

The test comprises 84 color caps stored in four wooden trays. On the back of each cap is a number from 1 to 84 indicating its correct position in the hue sequence around the Munsell color circle. Source: http://www.creativepro.com/files/story_images/img_8699.jpg. 


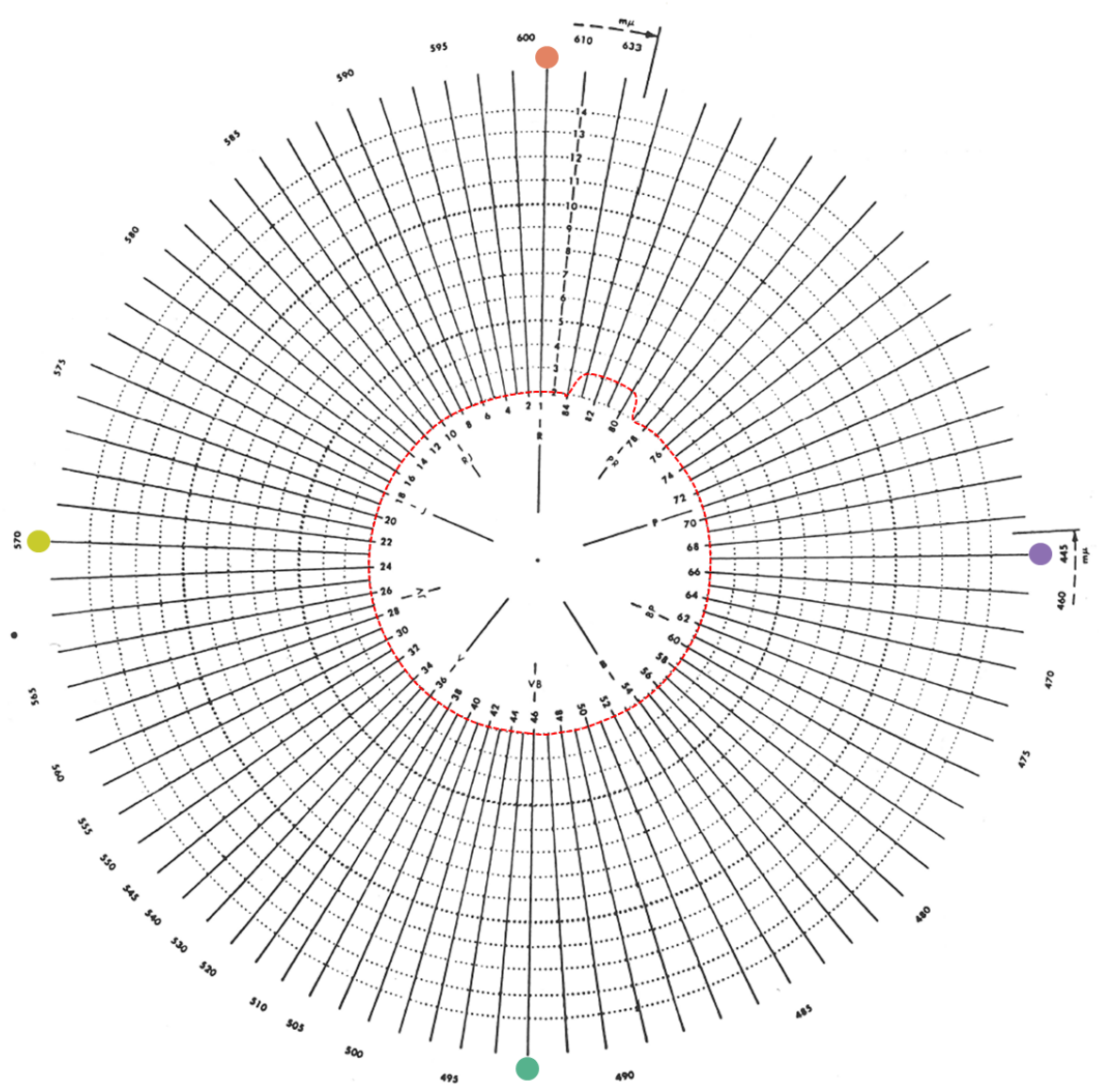

Figure 2.2: Results of the color discrimination test plotted on the Farnsworth-Munsell 100-Hue Test chart for a representative color-normal subject

In the chart, the central disk represents the entire color circle. Concentric rings are located outside the central ring at error score intervals of 1 . A perfect score of 2 per cap denotes that a participant had a superior competence for color discrimination along the hue circle. Error scores for a single subject are plotted in red along the hue circle. This subject made some errors close to number 82 , which corresponds to a hue of purplish red. 


\subsection{Experimental setup}

\subsubsection{Visual stimulus generator and display system}

For retinotopic mapping experiments, the visual stimuli were created using a stand-alone software tool based on the Microsoft DirectX library (StimulDX, Brain Innovation, Maastricht, The Netherlands). For the spatial frequency and temporal frequency experiments, stimuli were generated using a VSG ViSaGe system (Cambridge Research Systems Ltd., Rochester, UK) and the CRS VSG Toolbox for MATLAB ${ }^{\mathrm{TM}}$. A trigger pulse received from the MR-scanner was used to synchronize presentation of the visual stimuli with the fMRI experiments. All stimuli were presented projecting the image by a SANYO, PLC-XT 11 LCD projector (pixel resolution $1024 \times 768$, frame rate $80 \mathrm{~Hz}$ ) onto a translucent screen mounted on the top of the MRI head-coil visible by the subject through a $45 \mathrm{deg}$ tilted mirror (Schäfter \& Kirchhoff, Hamburg, Germany). The display screen subtended a visual angle of approximately 28 deg horizontally and 21 deg vertically. Light entered the scanner room through a tunnel in the scanner room's wall that was designed, by its length and diameter, for filtering out radiofrequency (RF) waves which might interfere with scanner frequencies. The configuration of the fMRI experimental setup is illustrated schematically in Figure 2.3. 


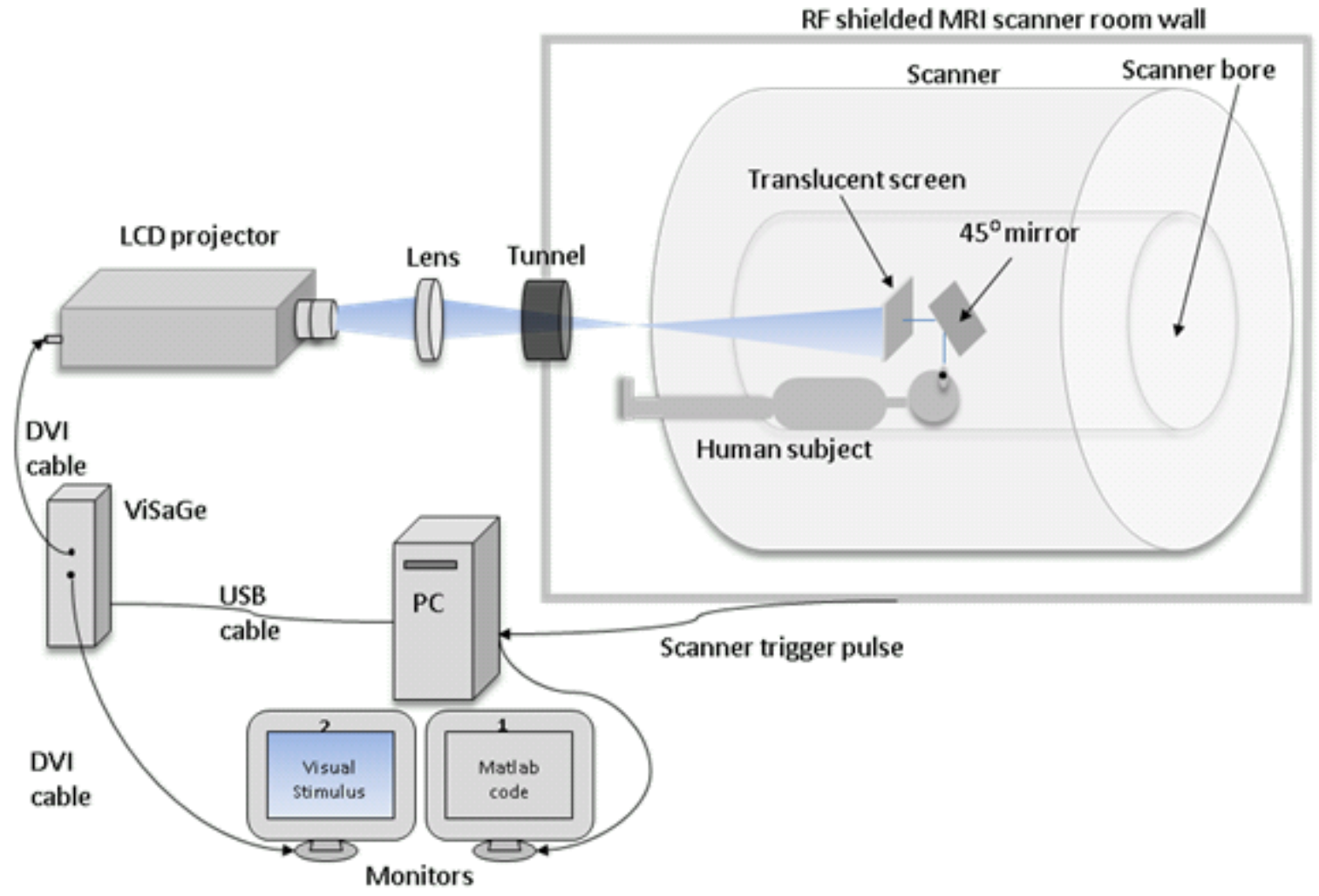

Figure 2.3: Schematic illustration of the fMRI experimental configuration

Visual stimuli were projected using an LCD projector (one inch LCD panel). A customized achromatic doublet lens was placed several inches away from the projector, in the path of the light, to focus the beam of light through a tunnel in the scanner room's wall onto the stimulus projection screen in the magnet bore. The tunnel, designed specifically for the transmission of light, filters radiofrequency (RF) waves which might interfere with scanner frequencies.

\subsubsection{Calibration of the LCD projector}

The LCD projector system was calibrated for luminance and chromaticity by defining gamma curves and spectral properties separately for the three primary color channels. Gamma correction refers to a procedure that captures and corrects the nonlinearity between the digital input value and output light intensity (luminance) level of a display (Bach et al., 1997; Brainard et al., 2002). The disadvantage of the standard luminance meters is that they do not function near an MRI scanner. We therefore used a fiber optic cable and a digital luminance meter (Mavo-Monitor USB, GOSSEN Foto- und Lichtmesstechnik GmbH, 
Nürnberg, Germany) to determine the relationship between the digital input value and the output luminance level of our LCD projector (Strasburger et al., 2002). One end of the cable was held against the display screen at the scanner where the observer viewed the stimulus, and the other end was connected to the luminance meter, which connected through a USB port to a PC housing the VSG stimulus generator. Special mechanical adaptors were used at the ends of the fiber optic cable to standardize and minimize the attenuation of light during the calibration process. Prior to gamma correction we determined the attenuation factor of the fiber optic to light energies of the three primary colors (Red (R), Green (G), and Blue (B)), and these values were used in subsequent steps to scale the measured luminance levels. The luminance values were entered manually into the VSG calibration software; and red, green, blue and white luminance outputs were subsequently calibrated (gamma corrected) by taking several readings for each primary color. These configurations were then saved in a calibration file that is loaded automatically whenever the VSG program initializes the ViSaGe hardware.

The chromaticity of the projector output (Kwak \& MacDonald, 2000) was calibrated with a PR-650 Spectra Colorimeter (PhotoResearch Inc., Chatsworth, MA). To allow measurement of the spectral emissions of the primary colors outside the scanner room, we placed a surface silver-coated mirror in the projector's light path at an angle of $45 \mathrm{deg}$; light directed with this setup was then made to fall onto a translucent screen outside the magnet room, thus enabling measurement of the spectra. The CIE chromaticity coordinates (Billmeyer \& Webber, 1953; Wyszecki, 1954; Chickering, 1969; Galbraith \& Marshall, 1985; Neri, 1990) for the R, G, and B colors obtained from the PR-650 were then entered into the VSG software before the gamma corrected look-up-tables (LUTs) were generated.

To study the temporal characteristics of the LCD projector we used a photodiode in combination with an operational amplifier, and a digital oscilloscope (TDS 2024B, Tektronix, Beaverton, OR) (Liu \& Wandell, 2005b). The rise and fall time of the LCD projector were sufficiently fast to present visual stimuli at temporal contrast modulation frequencies up to $12 \mathrm{~Hz}$. 


\subsection{Retinotopic mapping experiments}

\subsubsection{MRI data acquisition and processing}

All imaging studies for the retinotopic experiments were performed on a $2.9 \mathrm{~T}$ scanner (Siemens Tim Trio, Erlangen, Germany) using a 12-channel receive-only phased-array head coil in combination with the whole-body coil for radiofrequency (RF) pulse transmission.

Anatomical data: At the beginning of each retinotopic mapping session, T1-weighted MR images using a 3D MPRAGE sequence (Haase et al., 1989; Mugler \& Brookeman, 1990) at $1.3 \times 1 \times 1.3 \mathrm{~mm}^{3}$ resolution were acquired. These high-resolution anatomical images were used for reconstruction of the cortical surface in each subject. The surfaces were later used for overlaying activation maps, delineation of visual areas, as well as for defining region of interest (ROIs).

Functional data: A T2*-sensitive single-shot gradient-echo-planar-imaging (EPI) technique (Mansfield \& Maudsley, 1977; Ogawa et al., 1992) with an in-plane resolution of 2 $\times 2 \mathrm{~mm}^{2}$ (repetition time (TR): $2000 \mathrm{~ms}$, echo time (TE): $36 \mathrm{~ms}$, flip angle: $70 \mathrm{deg}$, acquisition matrix: $96 \times 96$ ) was used to acquire functional volumes. Twenty-two consecutive slices of 4 $\mathrm{mm}$ thickness approximately perpendicular to the calcarine fissure covering striate as well as the extrastriate visual areas in the occipital lobe were acquired.

Data processing: All data analyses were performed using BrainVoyager QX 1.10 software (Brain Innovation, Maastricht, The Netherlands). Standard procedures within BrainVoyager QX were used to segment white and gray matter and so identify the cortical boundary. The boundary is then inflated to give a smooth representation of the cortical surface which allows projection of the statistical maps of functional activation (Figure 2.4). 


\section{Materials and Methods}

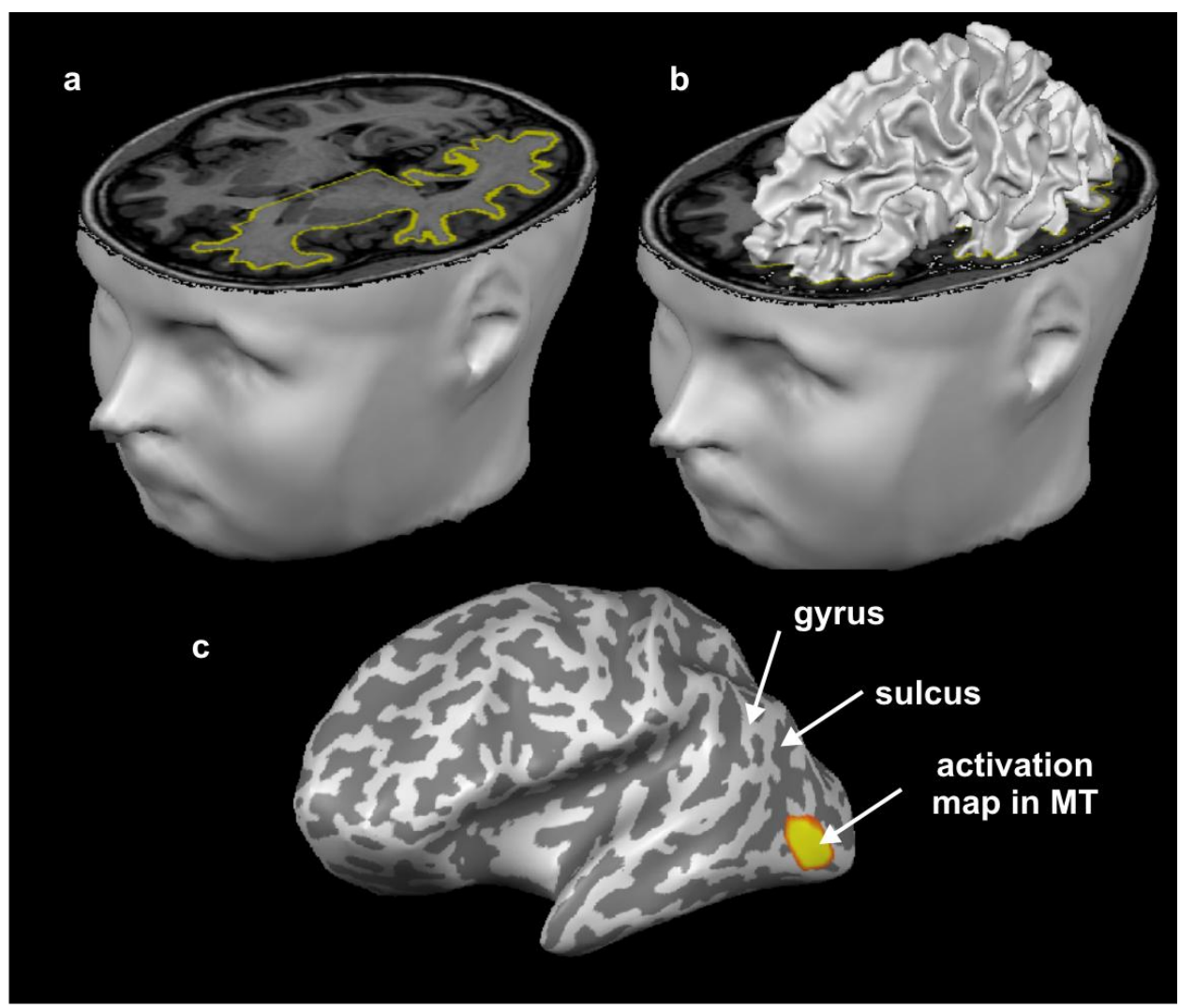

Figure 2.4: Reconstruction of the cortical surface

a) 3D reconstruction of the head of a representative subject. The boundary between the gray and the white matter is shown in yellow (as determined automatically in BrainVoyager QX). b) Reconstructed cortical surface for the left hemisphere. c) Inflated cortical surface. The sulci and gyri are represented in dark and gray shadings, respectively. Activation maps are overlaid on the inflated cortical surface (lateral views). MT is activated in response to moving-dot-pattern stimuli. 
Preprocessing of the functional data included deletion of a few initial volumes (to allow longitudinal magnetization to reach a steady state), 3D-motion correction (also including intra session alignment), slice-time correction, temporal high-pass filtering (3 cycles/run), linear trend removal, and spatial smoothing with a Gaussian kernel (full width at half maximum $4 \times 4 \times 4 \mathrm{~mm}^{3}$ ). After the preprocessing was completed, functional data were coregistered to the anatomical volume (MPRAGE) acquired at the beginning of the same session, and subsequently transformed into Talairach standard space (Talairach \& Tournaux, 1988). For further analyses, the Talairach transformed functional data sets were used. Detailed descriptions of the BrainVoyager data processing methods are available under: http://www.brainvoyager.com/bvqx/doc/UsersGuide/WebHelp/BrainVoyagerQXUsersGuide.htm

\subsubsection{Visual stimuli, experimental design, and data analysis}

\section{LGN localization}

For identification of LGN, a high-contrast ( $100 \%$ Michelson contrast) checkerboard stimulus was used (Kastner et al., 2004; Mullen et al., 2008). A fixation cross was presented at the center of the stimulus. The stimulus subtended a visual angle of 10.5 deg (radius) both horizontally and vertically. The spatial frequency and size of the checkerboard pattern was scaled by the human cortical magnification factor to activate the LGN approximately equivalently at all stimulated eccentricities. The spatial layout and timing of presentation of the stimulus is illustrated in Figure 2.5. In the experiment, the subjects' task was to maintain fixation at the center fixation cross and passively view the stimulus. The stimulus was presented using a block design comprising alternating blocks of $12 \mathrm{~s}$ stimulation and $18 \mathrm{~s}$ controls. The control block was a neutral gray background with luminance equal to the mean luminance of the checkerboard patterns. In the activation block, the contrast of the stimulus was reversed at a temporal frequency of $8.33 \mathrm{~Hz}$. The functional run (total length: 270 s) comprised eight cycles (one cycle = activation block + control block). In addition, a control block of $30 \mathrm{~s}$ was present at the start of the run. The data from the first $12 \mathrm{~s}$ (six volumes) of the control block, during which the magnetic field has to fully reach a steady state, was discarded in the analysis. General linear modeling (GLM) analysis of the data was performed for the individual subject to obtain activation maps in response to checkerboard 
stimulation. Statistical maps of t-values were visualized (Figure 2.6) at Bonferroni-corrected threshold level of $p<0.001$. ROls were constructed based on the statistically significant voxels. Further, it was found that the location of the functional maps corresponds well with the anatomical location of the LGN (Horton et al., 1990; Tamraz, 1994). The Talairach coordinates (Talairach \& Tournaux, 1988) for six subjects are given in Table 2.1.

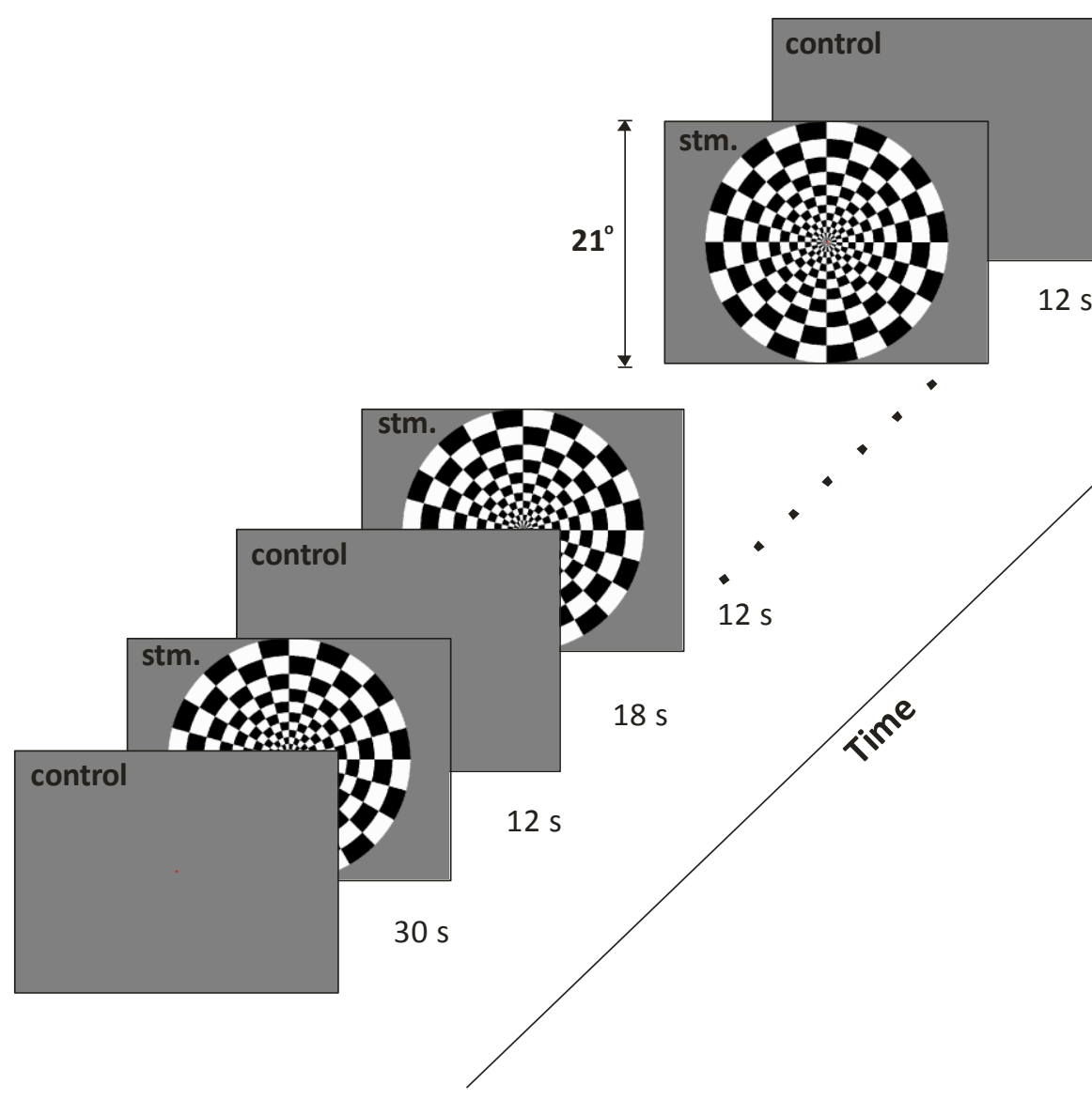

Figure 2.5: Spatial layout and timing of the LGN localizer stimulus

The high-contrast checkerboard stimulus subtended a visual angle of $10.5 \mathrm{deg}$ (radius). A central fixation cross was present at the center of the screen. The stimulus block (12 s) comprised flickering contrast patterns at $8.33 \mathrm{~Hz}$ flicker rate. In the control block (18 s) only a gray background was shown. Each functional run (270 s) comprised eight cycles (one cycle = activation block + control block). In addition, a control block of $30 \mathrm{~s}$ was used at the start of the run. 


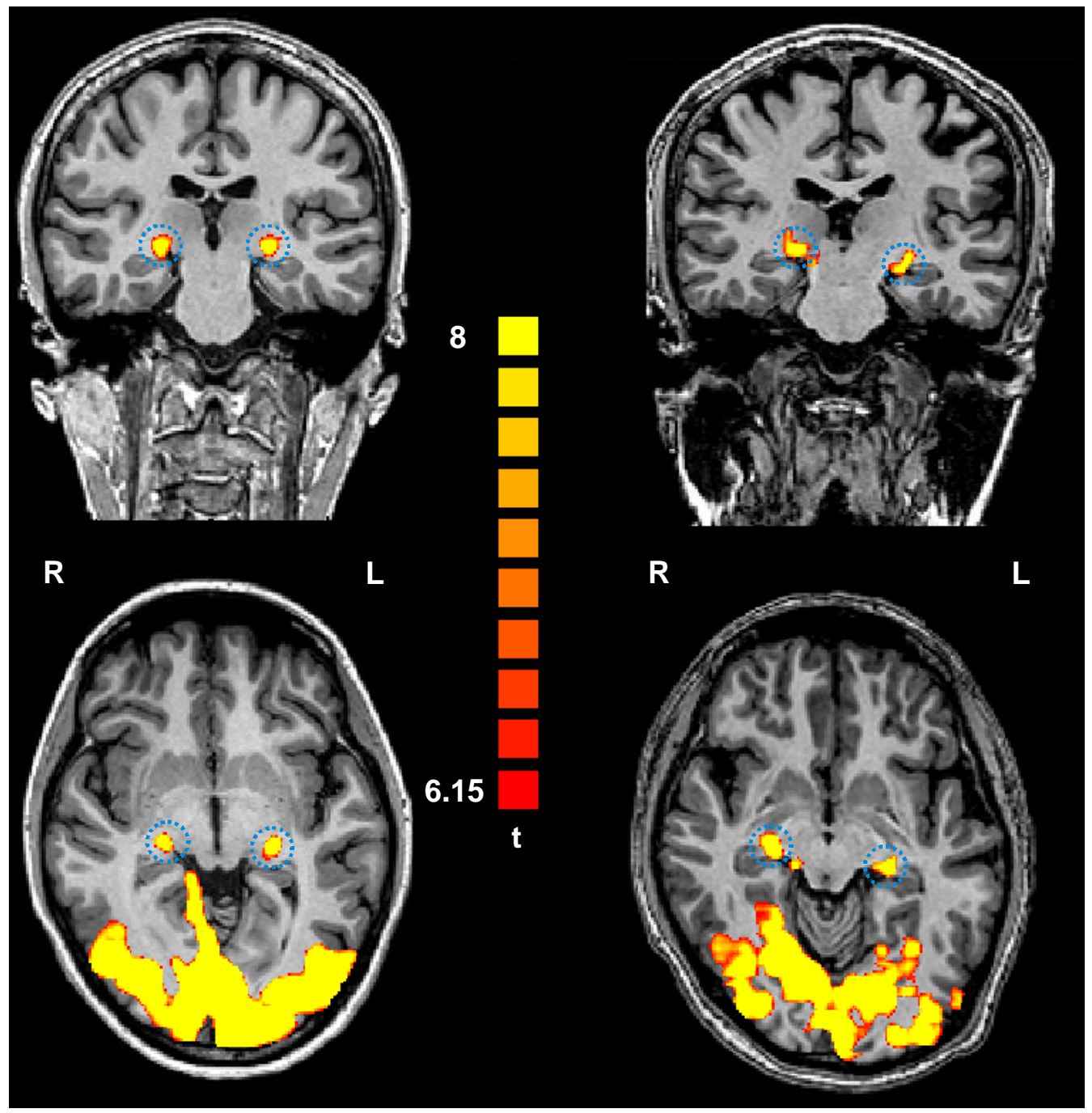

Figure 2.6: Functional activation of the lateral geniculate nucleus (LGN)

Activation patterns to the checkerboard stimulus are overlaid on structural images of two representative subjects (left and right columns, respectively). For each subject, activated regions are shown in coronal (first row) and axial (second row) planes. The dashed blue circles highlight LGNs. Scale indicates t-values of functional activity in colored regions. L: left hemisphere; R: right hemisphere. 


\begin{tabular}{|c|c|c|c|c|c|c|c|c|}
\hline \multirow[b]{3}{*}{ Subject } & \multicolumn{4}{|c|}{ Left LGN } & \multicolumn{4}{|c|}{ Right LGN } \\
\hline & \multicolumn{3}{|c|}{ TAL Coordinates $(\mathrm{mm})$} & \multirow{2}{*}{$\begin{array}{c}\text { Number } \\
\text { Of } \\
\text { voxels }\end{array}$} & \multicolumn{3}{|c|}{ TAL Coordinates $(\mathrm{mm})$} & \multirow{2}{*}{$\begin{array}{c}\text { Number } \\
\text { Of } \\
\text { voxels }\end{array}$} \\
\hline & $x$ & Y & z & & $x$ & y & Z & \\
\hline $\mathrm{DD}$ & -20 & -23 & -3 & 338 & 21 & -24 & -2 & 199 \\
\hline IB & -21 & -26 & -2 & 208 & 17 & -28 & -1 & 175 \\
\hline MR & -24 & -28 & 1 & 423 & 20 & -26 & 3 & 119 \\
\hline MT & -20 & -25 & 0 & 145 & 13 & -25 & 1 & 208 \\
\hline $\mathrm{MH}$ & -22 & -22 & -5 & 361 & 16 & -26 & -6 & 390 \\
\hline NJ & -22 & -28 & -3 & 293 & 19 & -24 & -1 & 184 \\
\hline Mean & -22 & -26 & -2 & 294 & 17 & -26 & -1 & 212 \\
\hline SD & 1 & 2 & 2 & 102 & 2 & 1 & 3 & 92 \\
\hline
\end{tabular}

Table 2.1: LGN coordinates

Talairach coordinates (Talairach \& Tournaux, 1988) and estimated numbers of voxels for six subjects.

\section{MT localization}

Stimuli were moving or stationary white-dot patterns presented on a gray background (Zeki et al., 1991; Watson et al., 1993; Tootell et al., 1995; Huk et al., 2002). The spatial layout and timing of presentation of the stimulus is illustrated in Figure 2.7. A fixation cross was present at the center of the stimulus. The stimuli were presented using a block design paradigm comprising blocks of $12 \mathrm{~s}$ stimulation (moving or stationary dots) and $12 \mathrm{~s}$ controls. The control block was a neutral gray background. Each functional run (total length: $204 \mathrm{~s}$ ) comprised four cycles (one cycle = moving dots block + control + stationary dots block + control). In addition, a control block of $12 \mathrm{~s}$ was used at the start of the run. The data from the first $12 \mathrm{~s}$ of the control block during which the magnetic field has to fully reach a steady state was discarded later in the analysis. GLM analysis was performed on the data to localize MT. Model regressors were created on the basis of the alternating moving- and static-dot blocks. Activation maps were created by contrasting the moving dot block with the stationary dot block. Statistical maps of t-values were visualized (Figure 2.8) at the Bonferroni corrected threshold level of $p<0.001$. ROls were constructed based on the statistically significant voxels. The Talairach coordinates (Talairach \& Tournaux, 1988) for six subjects are given in Table 2.2 . 


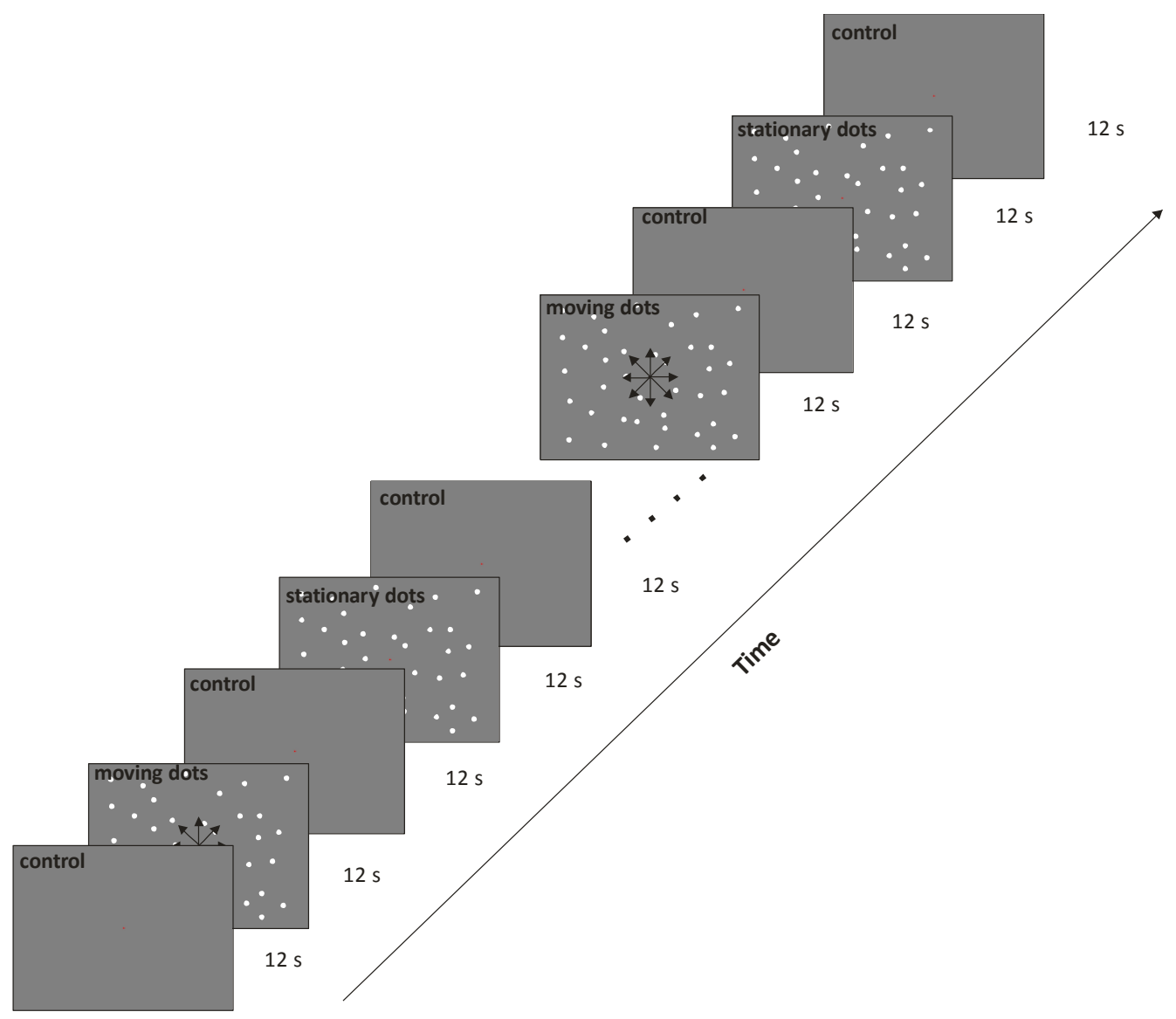

Figure 2.7: Spatial layout and timing of the MT localizer stimulus

Moving and stationary dot patterns were presented in a block design. A fixation cross was present at the center of the stimulus. The stimuli were presented using a block design comprising blocks of $12 \mathrm{~s}$ stimulation (moving or stationary dots) or and $12 \mathrm{~s}$ controls. The control block was a neutral gray background. Each functional run $(204 \mathrm{~s}$ ) comprised four cycles (one cycle = moving dots block + control block + stationary dots block + control). In addition, a control block of $12 \mathrm{~s}$ was used at the start of the run. 


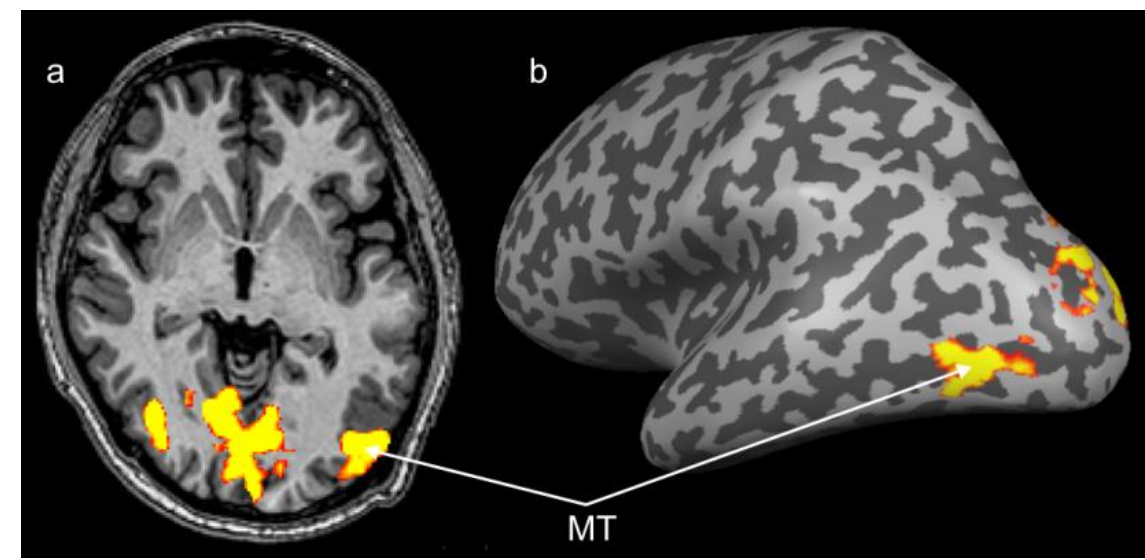

Figure 2.8: Functional activation of the MT

Activation map obtained from the MT localizer task is shown a) on an axial plane b) on the reconstructed cortical surface (left hemisphere, lateral view) of a representative subject.

\begin{tabular}{|c|c|c|c|c|c|c|c|c|}
\hline \multirow[b]{3}{*}{ Subject } & \multicolumn{4}{|c|}{ Left MT } & \multicolumn{4}{|c|}{ Right MT } \\
\hline & \multicolumn{3}{|c|}{ TAL Coordinates $(\mathrm{mm})$} & \multirow{2}{*}{$\begin{array}{c}\text { Number } \\
\text { Of } \\
\text { voxels }\end{array}$} & \multicolumn{3}{|c|}{ TAL Coordinates $(\mathrm{mm})$} & \multirow{2}{*}{$\begin{array}{c}\text { Number } \\
\text { Of } \\
\text { voxels }\end{array}$} \\
\hline & $x$ & $y$ & $z$ & & $x$ & $y$ & $z$ & \\
\hline $\mathrm{DD}$ & -46 & -64 & 5 & 1310 & 45 & -69 & -2 & 1218 \\
\hline IB & -44 & -62 & -2 & 1265 & 43 & -55 & -2 & 963 \\
\hline MR & -40 & -69 & 5 & 1569 & 40 & -64 & 2 & 975 \\
\hline MT & -39 & -70 & -2 & 226 & 39 & -67 & -1 & 277 \\
\hline $\mathrm{MH}$ & -42 & -66 & 1 & 472 & 43 & -66 & -1 & 857 \\
\hline NJ & -41 & -76 & 1 & 1127 & 36 & -67 & -1 & 590 \\
\hline Mean & -42 & -68 & 1 & 994 & 41 & -65 & -1 & 813 \\
\hline SD & 2 & 4 & 3 & 526 & 3 & 5 & 1 & 332 \\
\hline
\end{tabular}

Table 2.2: MT coordinates

Talairach coordinates (Talairach \& Tournaux, 1988) and estimated numbers of voxels are shown for six subjects.

\section{Retinotopic mapping}

Retinotopy links the position of each cortical neuron to the point in the visual field corresponding to the center of its receptive field. Adjacent neurons represent adjacent points in the visual field (Warnking et al., 2002). Thus, the position of neurons can be best described by two orthogonal dimensions of the visual field, namely polar angle and eccentricity. Several retinotopically organized visual areas are present in the visual cortex 
which abut one another at the representation of the horizontal (polar angle $=0$ deg and 180 deg) and vertical meridians (polar angle $=90 \mathrm{deg}$ and $270 \mathrm{deg}$ ) of the visual field.

\section{Eccentricity mapping}

Visual field eccentricities were mapped using high-contrast reversing $(8.33 \mathrm{~Hz})$ annular checkerboard ring stimuli. The size and spatial frequency of ring stimuli were scaled to activate an approximately equivalent area of visual cortex at all stimulated eccentricities. The spatial layout and timing of presentation of the stimulus are illustrated in Figure 2.9. In the experiment, the subjects' task was to maintain fixation at the central fixation cross and to passively view the stimulus. Six ring stimuli with increasing annular size were presented sequentially in blocks of $10 \mathrm{~s}$ durations. Each functional run (total length: $370 \mathrm{~s}$ ) comprised six cycles (one cycle = six activation blocks, each comprising a different ring stimulus). In addition, a control block of $10 \mathrm{~s}$ was used at the start of the run, and the data was discarded in the analysis. For the detection of activation maps a cross-correlation analysis was performed (Linden et al., 1999; Lu et al., 2005). Cross-correlation analysis can be used for identifying the time point (lag) at which an eccentricity responds maximally. A series of cross correlation coefficients were calculated between the signal time-course and temporally shifted stimulus paradigm. The activation map was then color coded (Figure 2.10) according to the phase shift at which the maximum cross-correlation coefficient was achieved. This coding scheme can distinguish cortical regions corresponding to different visual field eccentricities. Eccentricity maps were also confirmed by traveling wave stimuli (DeYoe et al., 1994; Engel et al., 1994; Sereno et al., 1995; Engel et al., 1997b; Tootell et al., 1998; Wandell, 1999; Warnking et al., 2002). 


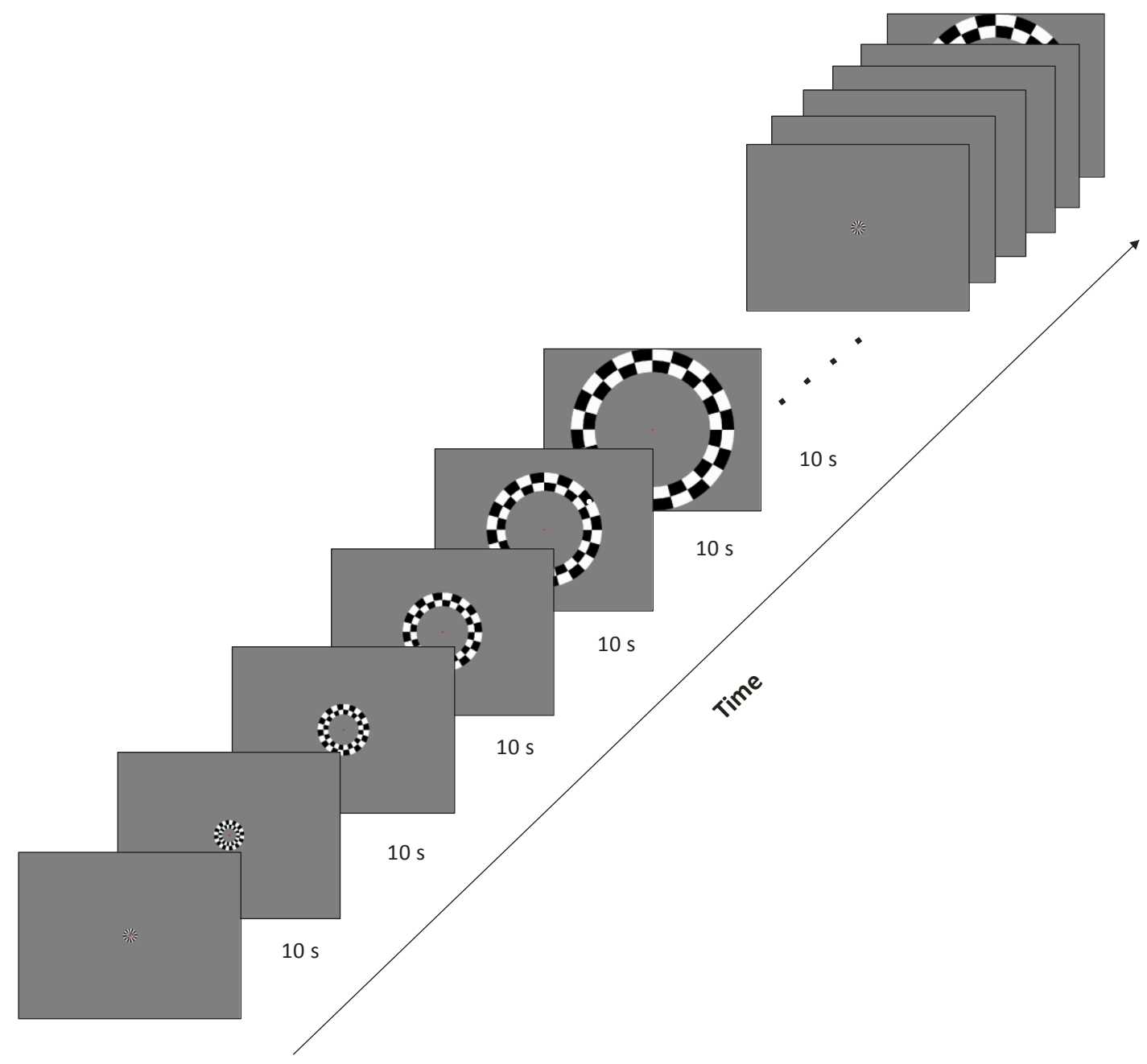

Figure 2.9: Spatial layout and timing of eccentricity mapping stimulus

Annular ring stimuli with increasing eccentricity were presented. The stimuli were presented using a block design comprising blocks of $10 \mathrm{~s}$ stimulation (for each ring). Each functional run (370 s) comprised six cycles (one cycle = six eccentricity rings). In addition, a control block of $10 \mathrm{~s}$ was used at the start of the run. 


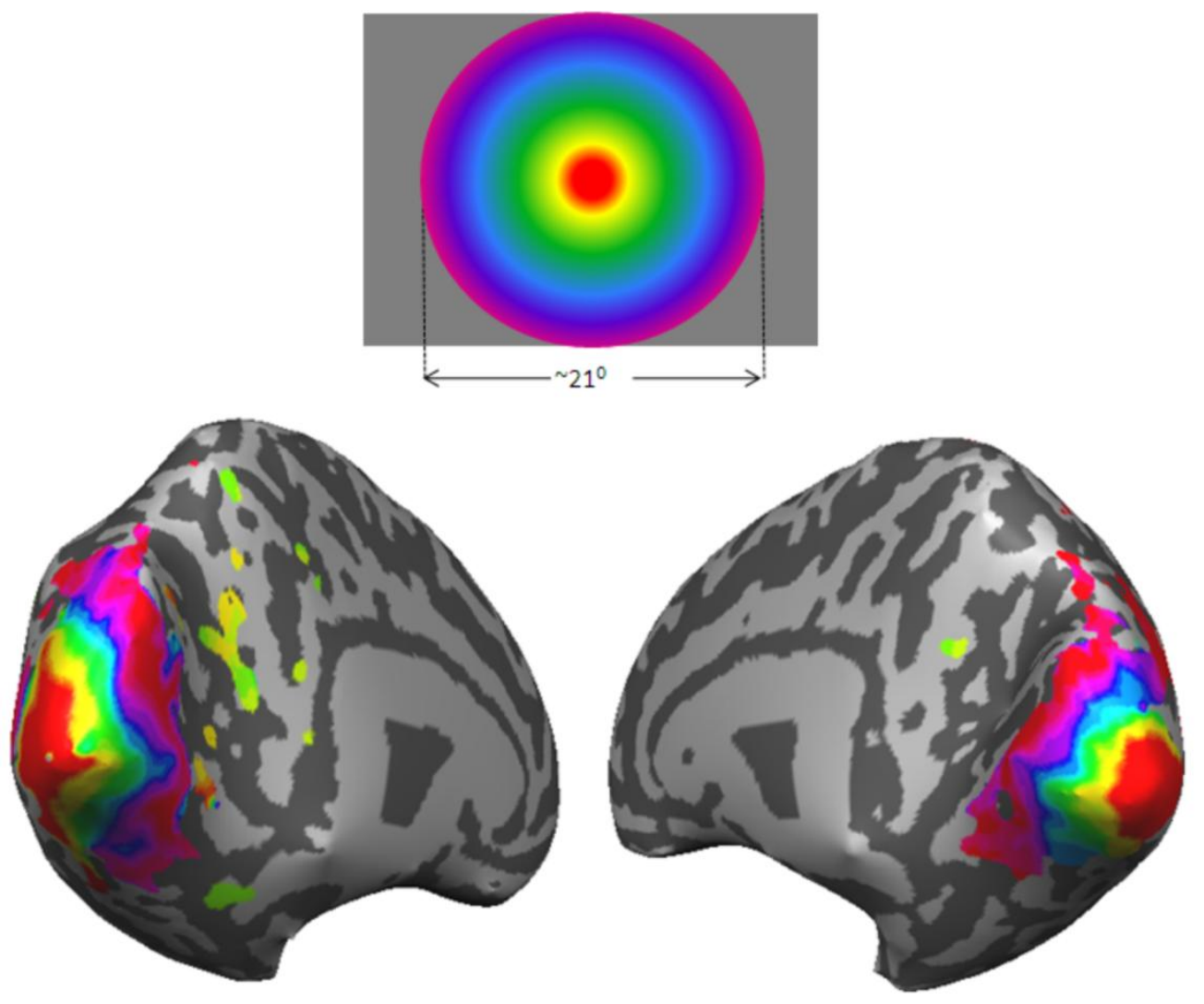

Figure 2.10: Eccentricity maps

Eccentricity maps are displayed on reconstructed cortical surfaces of the left and right hemispheres of a representative subject. An annular ring stimulus with increasing diameter (pseudocolor map: red to violet) results in a traveling wave of activation spreading from the occipital pole to more anterior parts of the brain. The maximal eccentricity measured was $10.5 \mathrm{deg}$ (radius).

\section{Meridian mapping}

Visual field meridians were mapped using high-contrast reversing $(8.33 \mathrm{~Hz})$ horizontal and vertical wedge (width $30 \mathrm{deg}$ ) stimuli. Such stimuli result in two activation patterns, corresponding to the horizontal and vertical meridians. Owing to the intrinsic mirror representation of the visual field in early visual areas, it is known that the meridian representations of each activation pattern specify the boundaries between adjacent visual areas (Sereno et al., 1994). The spatial layout and timing of stimulus presentation is illustrated in Figure 2.11. In the experiment, the subjects' task was to maintain fixation at the central fixation cross and to passively view the stimulus. Horizontal and vertical meridian stimuli were presented alternately in blocks of $12 \mathrm{~s}$ durations. Each functional run (total length: $312 \mathrm{~s}$ ) comprised twelve cycles (one cycle = horizontal meridian + vertical meridian). 


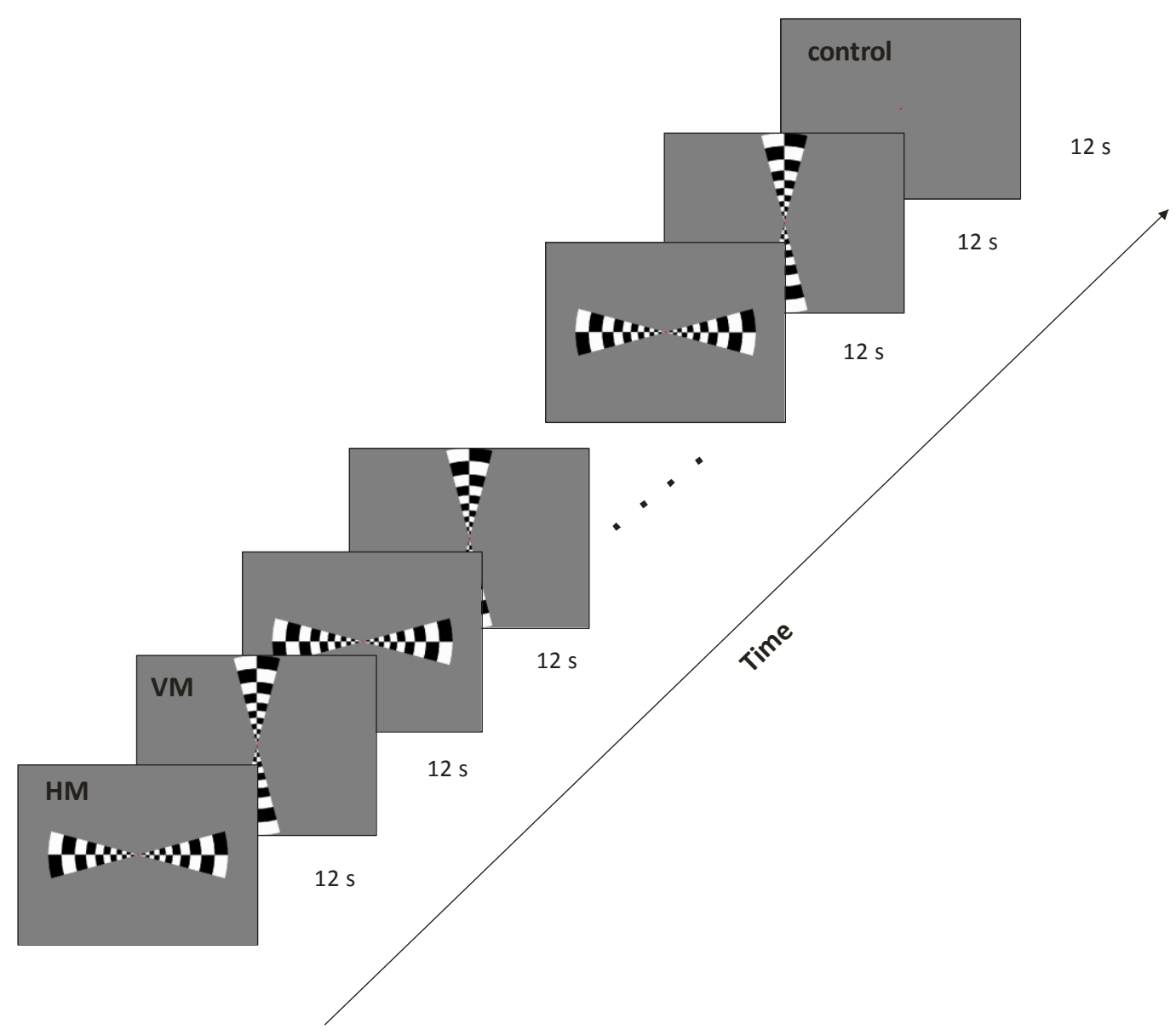

Figure 2.11: Spatial layout and timing of meridian mapping stimulus

Horizontal (HM) and vertical meridian (VM) wedge stimuli were presented alternatively in blocks of $12 \mathrm{~s}$ duration. The functional run (312 s) comprised twelve cycles (one cycle = horizontal-wedge block + verticalwedge block). In addition, a control block of $12 \mathrm{~s}$ was used at the beginning and end of the run. 
In addition, a control block of $12 \mathrm{~s}$ was used at the start and end of each run, and the data of the first 12 seconds of the experiment were discarded in the analysis. For detection of activation maps we performed a cross-correlation analysis (Linden et al., 1999; Lu et al., 2005). A series of cross-correlation coefficients were calculated between the signal timecourse and the temporally shifted stimulus paradigm. The activation map was then color coded (Figure 2.12) according to the phase shift at which the maximum cross-correlation coefficient was achieved. This coding scheme can distinguish cortical regions corresponding to the two meridian wedge stimuli, hence establishing borders between different visual areas. Similarly, we also used the standard rotating wedge stimuli for polar angle measurements, for confirmation of the exact location of the visual area boundaries (DeYoe et al., 1994; Engel et al., 1994; Sereno et al., 1995; Engel et al., 1997b; Warnking et al., 2002). The mean Talairach (Talairach \& Tournaux, 1988) coordinates (for six subjects) of the mapped visual areas are shown in Table 2.3. 


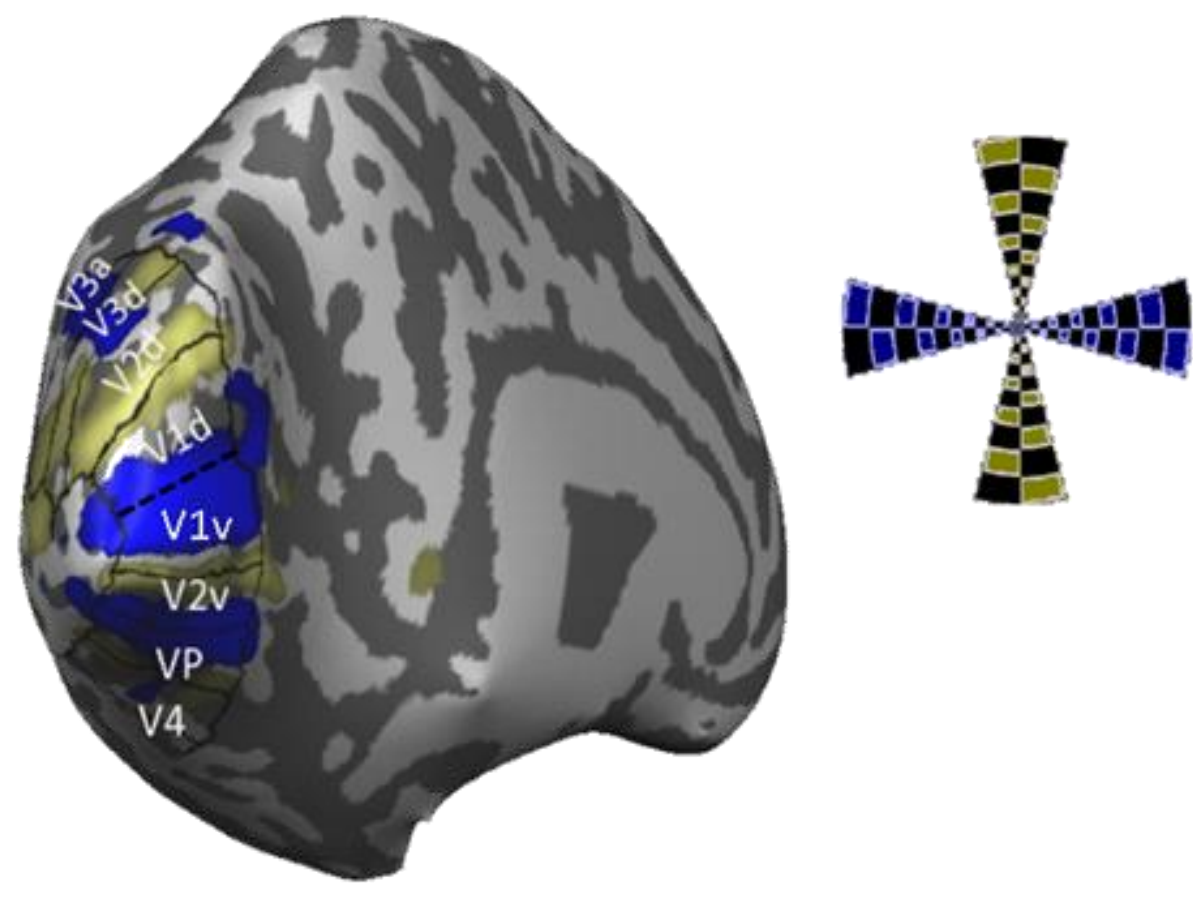

Figure 2.12: Meridian maps

Activation maps in response to horizontal (blue color) and vertical (golden color) meridian stimuli are displayed on reconstructed cortical surfaces for the left hemisphere of a representative subject. The borders between visual areas are demarcated.

\begin{tabular}{|c|c|c|c|c|c|c|c|c|}
\hline \multirow[b]{3}{*}{ Area } & \multicolumn{4}{|c|}{ Left hemisphere } & \multicolumn{4}{|c|}{ Right hemisphere } \\
\hline & \multicolumn{3}{|c|}{ TAL Coordinates (mm) } & \multirow{2}{*}{$\begin{array}{c}\text { Number } \\
\text { Of } \\
\text { voxels }\end{array}$} & \multicolumn{3}{|c|}{ TAL Coordinates (mm) } & \multirow{2}{*}{$\begin{array}{c}\text { Number } \\
\text { Of } \\
\text { voxels }\end{array}$} \\
\hline & $x$ & y & z & & $x$ & y & z & \\
\hline V1 & -8 & -86 & 1 & 5336 & 8 & -83 & 3 & 4940 \\
\hline $\mathrm{V} 2 \mathrm{v}$ & -11 & -78 & -9 & 2497 & 8 & -77 & -7 & 2269 \\
\hline V2d & -13 & -94 & 12 & 2900 & 13 & -89 & 12 & 2751 \\
\hline VP & -19 & -73 & -10 & 2063 & 16 & -71 & -8 & 2117 \\
\hline V3d & -23 & -88 & 10 & 2129 & 22 & -86 & 12 & 1997 \\
\hline V3a & -30 & -83 & 7 & 1730 & 30 & -80 & 6 & 1842 \\
\hline V4 & -27 & -68 & -11 & 1732 & 24 & -68 & -10 & 1900 \\
\hline
\end{tabular}

Table 2.3: Coordinates for visual areas

Average Talairach (Talairach \& Tournaux, 1988) coordinates (six subjects) and estimated numbers of voxels are shown for retinotopically mapped visual areas. 


\subsection{Definition of the chromatic and luminance pathway selective stimuli}

Three types of stimuli, namely "L-M", " $L+M$ ", and " $S$ " were designed to individually stimulate the L-M cone-opponent (red-green), luminance, and S-cone (blue-yellow) pathways, respectively. The L-M and S stimuli were isoluminant, i.e. varied in their chromatic content only (isoluminant chromatic stimuli), whereas in the L+M stimulus, only the luminance was modulated (isochromatic luminance stimulus). The chromaticity of the stimuli can be defined using a three dimensional cone-contrast space in which each axis represents the quantal catch of the L-, M-, and S-cone types, respectively, normalized with respect to a background color. Stimulus chromaticity is given by vector direction and contrast by vector length within that cone-contrast space. The space is designed to be device-independent and orthogonal with respect to post-receptoral pathway activation (Mullen et al., 2007). For example, a stimulus designed to elicit specific responses in the redgreen pathway will not activate the other two post-receptoral pathways (luminance and blue-yellow), although this is sometimes difficult to achieve in practice. In order to achieve equitable comparison between response properties in several visual areas at different eccentricities, originating from three post-receptoral pathways, the stimulus was made equal with regard to the initial coding stage. So the $L-M, L+M$, and $S$ stimuli in the experiments were designed such that the mean cone-contrast was equal in each case, i.e. the vectors defining the stimuli in the cone-contrast space had equal magnitudes for the three stimuli.

Selective stimulation of post-receptoral channels was achieved using red (R), green (G), and blue $(B)$ values for the LCD projector. These were derived using physiological criteria, which require knowledge of the spectral sensitivity of each cone type: S-, M- and L-cones (also known as "cone fundamentals" (Leonova \& Smithson, 2002)) as well as of the spectral profiles of the red, green, and blue lights produced by the LCD projector. For our calculations, the Stockman and Sharp (2000) 10 deg cone fundamentals (Figure 2.13), and the RGB spectra of the LCD projector as measured by a PR-650 spectroradiometer (Figure 2.14) were used. 


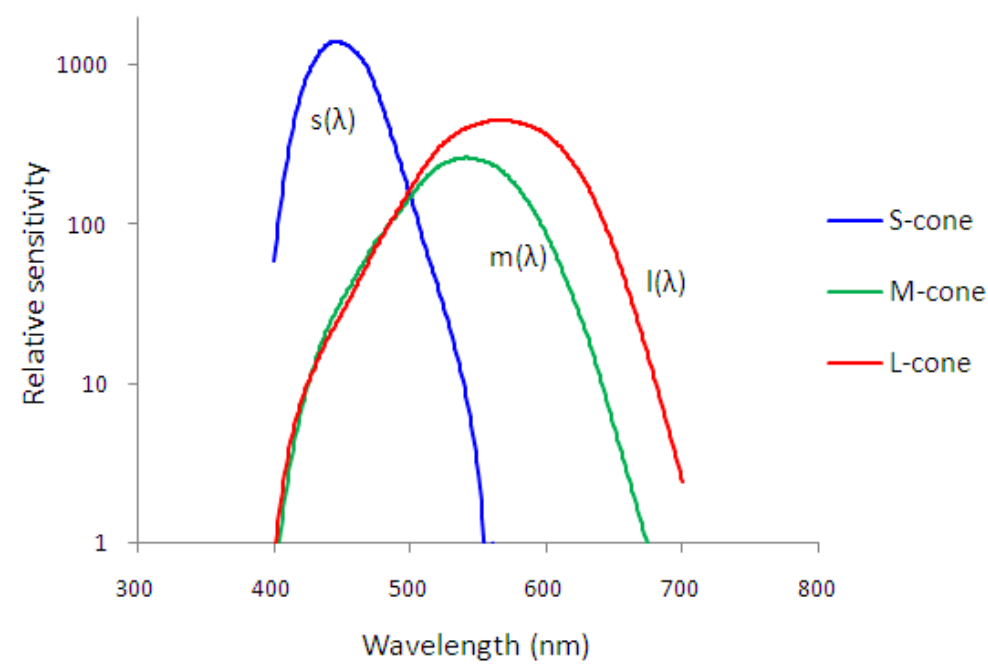

Figure 2.13: Human cone spectral sensitivities

The 10 deg cone fundamentals shown for S-, M-, and L-cones according to Stockman and Sharp (2000).

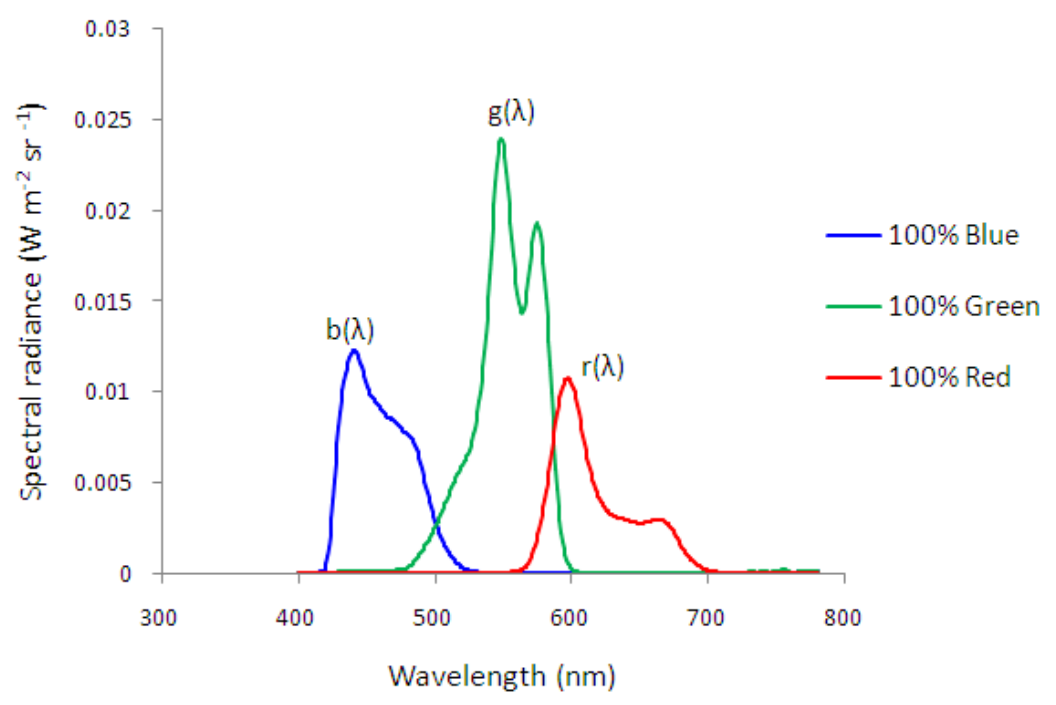

Figure 2.14: Spectral distribution of RGB lights

The spectral energy distributions of red, green and blue lights of the LCD projector, when displayed at their maximum intensities, were measured using the PR-650 spectroradiometer. Maximum intensities correspond to RGB values of [ [ $\left.\begin{array}{lll}1 & 1 & 1\end{array}\right]$. 
The matrix notations for the cone spectral sensitivities as well as for the RGB spectra are shown below:

$$
\text { Cone spectral sensitivities, } S=\left[\begin{array}{ccc}
s\left(\lambda_{400}\right) & m\left(\lambda_{400}\right) & l\left(\lambda_{400}\right) \\
\vdots & \vdots & \vdots \\
s\left(\lambda_{700}\right) & m\left(\lambda_{700}\right) & l\left(\lambda_{700}\right)
\end{array}\right] \text {, }
$$

where the columns contain the spectral sensitivities of the S-, M-, and L-cones, respectively at wavelength $\lambda_{i}(\mathrm{~nm})$ and

$$
\text { RGB spectra, } P=\left[\begin{array}{lll}
b\left(\lambda_{400}\right) & \cdots & b\left(\lambda_{700}\right) \\
g\left(\lambda_{400}\right) & \cdots & g\left(\lambda_{700}\right) \\
r\left(\lambda_{400}\right) & \cdots & r\left(\lambda_{700}\right)
\end{array}\right]
$$

where the rows contain the spectral density of the R-, G-, and B-light-emitting elements, respectively at wavelength $\lambda_{i}$ of the visual spectrum in steps of $4 \mathrm{~nm}$.

The maximum excitation produced by all three emitter systems in all three cone-types is the product of matrices $P$ and $S$. The resultant $3 \times 3$ matrix $E$ is, by definition,

$$
\begin{gathered}
\text { Cone excitation, } E=P \cdot S=\left[\begin{array}{ccc}
\sum_{\lambda} s(\lambda) * b(\lambda) & \sum_{\lambda} s(\lambda) * g(\lambda) & \sum_{\lambda} s(\lambda) * r(\lambda) \\
\sum_{\lambda} m(\lambda) * b(\lambda) & \sum_{\lambda} m(\lambda) * g(\lambda) & \sum_{\lambda} m(\lambda) * r(\lambda) \\
\sum_{\lambda} l(\lambda) * b(\lambda) & \sum_{\lambda} l(\lambda) * g(\lambda) & \sum_{\lambda} l(\lambda) * r(\lambda)
\end{array}\right] \\
E=\left[\begin{array}{lll}
S_{B} & S_{G} & S_{R} \\
M_{B} & M_{G} & M_{R} \\
L_{B} & L_{G} & L_{R}
\end{array}\right]
\end{gathered}
$$

In the above matrix $E$, each column describes maximum cone excitations caused by emission from one of the projector emitters. The excitations from the blue emitters are in the first column, and excitations due to the green and red emitter systems are in the second and third column, respectively. For further calculations, it is also necessary to calculate the maximum luminance of each emitter system. The luminance value $L U M$ of a light at any $R, G$ or $B$ value can be calculated as the integral over wavelength of the radiance of the light weighted with the luminosity function $V_{\lambda}$. This function describes the sensitivity of the human visual system to lights at wavelengths of the visible spectrum ( $400-700 \mathrm{~nm}$ ). It is generally assumed that the $V_{\lambda}$ function is a sum of the $\mathrm{M}$ - and L-cone excitations (i.e. the 
second and third row in the matrix). Thus, the maximum luminance values of our B-, G-, and R-emitters can be calculated from the cone excitation values from matrix $E$, and can be given as:

$$
\begin{aligned}
& L U M_{B}=M_{B}+L_{B} \\
& L U M_{G}=M_{G}+L_{G} \\
& L U M_{R}=M_{R}+L_{R}
\end{aligned}
$$

In the next sections, the steps involved in the calculation of RGB values required for the construction of pathway-selective stimuli are described.

\subsubsection{L-M cone-opponent (red-green) pathway selective stimulus}

The L-M cone-opponent pathway provides a neural substrate for the psychophysical redgreen color vision in humans. This pathway involves the differencing of signals from the Land M-cones. The "L-M" stimulus aimed to selectively stimulate the red-green pathway in the experiments comprised contrast reversal of red-green circular grating patterns (Figure 2.15).

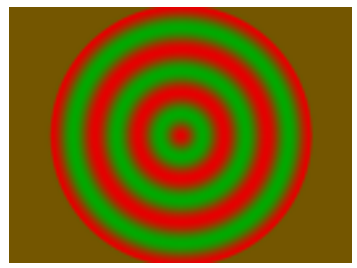

Figure 2.15: Example stimulus for isolation of the L-M cone-opponent (red-green) pathway

An ideal L-M stimulus would preferentially stimulate the red-green pathway and not activate the other two pathways, i.e. the luminance and blue-yellow pathways. The steps involved in deriving the required RGB values are described below.

First, the luminance of the red and green grating elements must be equal (isoluminance); failure to meet this requirement would result in an undesired stimulation of 
the luminance pathway due to a residual luminance signal. The equation which describes the equality of luminance between red and green grating patches is given as:

$$
r L U M_{R}+b L U M_{B}=g L U M_{G}
$$

Here, $r, g$, and $b$ are the required projector emitter values which are unknown. $L U M_{R}, L U M_{B}$, and $L U M_{G}$ are the maximum luminances of the projector R-, B-, and G-emitter systems as determined by summing the quantal excitations of $\mathrm{L}$ - and $\mathrm{M}$-cones as described above. In addition, each subject's isoluminance point was ascertained. Individuals may vary in their luminosity functions; the hypothesis that this variability is due to interindividual variability in the relative numerosity of the $\mathrm{L}$ - and $\mathrm{M}$-cones has now received experimental confirmation in experiments in which cone numerosity was directly assessed using adaptive optics (Hofer et al., 2005). The minimum motion technique (Cavanagh et al., 1984) was used to determine each subject's isoluminance points for the three lights of the projector. This technique involves an apparent motion stimulus, and relative luminance of two lights determines the direction of apparent motion. At isoluminance, little or no motion is apparent.

The stimulus was presented within the fMRI apparatus. Each subject set relative luminances of the red and blue relative to the green lights from the projector. The intention was to modify luminance values in the chromatic stimuli for each individual's isoluminance settings. It turned out that all subjects tested had luminosity functions close to the standard observer, so little or no correction was necessary.

Second, the contrast reversing red-green grating patterns should differentially excite only the L- and M-cones and should not modulate the S-cones, i.e. the amount of excitation produced in the S-cones by the red and green regions of the gratings must be equal. This condition can be stated as:

$$
r S_{R}+b S_{B}=g S_{G}
$$

with the same $r, g, b$ values as above; $S_{R}, S_{B}$, and $S_{G}$ are the maximum S-cone excitations produced by the projector RGB lights as obtained from the calibration matrix $E$. 
To achieve maximal activation of the L-M pathway, the $r$ value in equation (2.6.4) was set to a maximum value, i.e. $r=0.85$ (beyond this value the R-emitter intensity becomes strongly non-linear, as determined by the gamma correction procedure). In both equations (2.6.4) and (2.6.5), the blue term is added to the left side of the equation. This is done to cancel out the small amount of S-cone excitation produced by the green emitter. This combination was matched in luminance by the G-emitter. The G- and B-emitter values can be calculated from equation (2.6.4) and (2.6.5). The gratings varied in chrominance between two peaks, i.e. red ([0.85 00.006$])$ and green ([0 0.2 0 $]$ ), and the mean of these peaks results in a chrominance, which was used as the reference color. It is possible to express the modulation depth of the stimuli in terms of the contrast produced in individual cones, which is $\left(I_{\max }-I_{\min }\right) /\left(I_{\max }+I_{\min }\right)$, where $I$ is cone excitation. For example, for the red-green grating stimuli, the Michelson contrast generated in the L-cones $\left(C_{L}\right)$ is given by:

$$
\begin{gathered}
C_{L=}\left[\left(r_{\max } L_{R}+g_{\text {max }} L_{G}+b_{\text {max }} L_{B}\right)-\left(r_{\text {min }} L_{R}+g_{\text {min }} L_{G}+b_{\text {min }} L_{B}\right)\right] * 100 / \\
\left(r_{\text {max }} L_{R}+g_{\text {max }} L_{G}+b_{\text {max }} L_{B}\right)+\left(r_{\text {min }} L_{R}+g_{\text {min }} L_{G}+b_{\text {min }} L_{B}\right)
\end{gathered}
$$

here $r_{\text {max }}, g_{\max }, b_{\max }=\left[\begin{array}{lll}0.85 & 0 & 0.006\end{array}\right]$ and $r_{\min }, g_{\min }, b_{\min }=\left[\begin{array}{lll}0 & 0.2 & 0\end{array}\right]$ and $L_{R}, L_{G}$, and $L_{B}$ are the Lcone excitations from matrix $E$ (see above). The contrast values in the $\mathrm{S}$ - and $\mathrm{M}$-cones can be derived in the same way. The following contrast values were obtained, $C_{S}=0 ; C_{M}=27.19$; $C_{L}=10.56$; Thus, the L-M (red-green) stimulus is effective only to the L- and M-cones. In the experiments the stimulus contrast ("cone-contrast") is defined as the vector length (square root of the sum of squares) of individual cone contrasts.

Cone-contrast is defined as $\left(C_{S}^{2}+C_{M}^{2}+C_{L}^{2}\right)^{1 / 2}$. The cone-contrast value in case of the L-M stimulus was $29 \%$.

\subsubsection{L+M (luminance) pathway selective stimulus}

The $L+M$ pathway is thought to provide a neural substrate for the psychophysical luminance channel, and sums the responses from $L$ - and $M$-cones. The so-called " $L+M$ " stimulus aimed to selectively stimulate the luminance pathway comprised contrast reversing yellow-black circular grating patterns (Figure 2.16). 


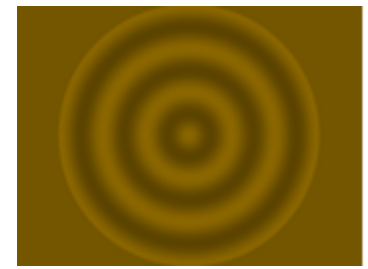

Figure 2.16: Example stimulus for isolation of $L+M$ (luminance) pathway

An L+M stimulus would preferentially stimulate the luminance pathway and be invisible to the other two pathways, i.e. the red-green and blue-yellow pathways. Unlike the L-M stimulus, the gratings were generated by in-phase summation of sinusoidal luminance modulations of the R- and G-emitters of the LCD projector. The resultant grating was uniform in chrominance (isochromatic) and at 100\% contrast appeared yellow ([0.85 0.2 0.006 $]$ ) and black ([ $\left.\left[\begin{array}{ll}0 & 0\end{array}\right]\right)$ at its modulation peaks. Luminance modulation was set to match the L-M cone contrast (29\%).

\subsubsection{S-cone (blue-yellow) pathway stimulus}

The S-cone pathway provides a neural substrate for psychophysical blue-yellow vision in primates. This pathway involves the differencing of the summed signal of L- and M-cones from the S-cones. The so called " $\mathrm{S}$ " stimulus in the experiments aimed to selectively stimulate the blue-yellow pathway comprised contrast reversing purple-green circular grating patterns (Figure 2.17).

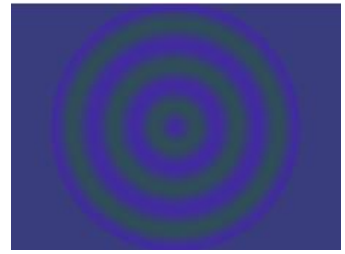

Figure 2.17: Example stimulus for isolation of S-cone (blue-yellow) pathway 
To arrive at the RGB values, first the luminance of the grating patches should be matched, which is given by

$$
r L U M_{R}+b L U M_{B}=g L U M_{G}
$$

Second, the contrast reversing grating patterns should differentially excite only the S cones and should be equally effective for the $\mathrm{M}$ - and L-cones, i.e. the amount of excitation produced in the M-cones by the purple and green patches must be equal. This condition can be described as:

$$
r M_{R}+b M_{B}=g M_{G}
$$

Here, $r, g$, and $b$ are again the unknown projector emitter values which are to be determined. $S_{R}, S_{B}$, and $S_{G}$ are the maximum S-cone excitations produced by the projector RBG lights as obtained from the calibration matrix $E$. By setting the $b$ value to 0.85 in equation (2.6.7), the remaining emitter values were calculated using equation (2.6.7) and (2.6.8) (as described previously in Section 2.6.1). The cone-contrast was scaled to match that of L-M and L+M stimuli (29\%). 


\subsection{Spatial frequency experiments}

\subsubsection{Visual stimuli}

This set of experiments was designed to study the influence of stimulus eccentricity and spatial frequency on activation in visual areas. The stimuli were contrast-reversing $(2 \mathrm{~Hz})$ circular sine-wave gratings (Mullen et al., 2007), spanning a visual angle of 21 deg (diameter) together with a cross (1 deg in diameter) at the center of the screen, which was used for the fixation task. Stimuli of various spatial frequencies $(0.27,0.55,1.09,2.2$, and 4.4 cpd) were used. In addition to using grating stimuli with a fixed spatial frequency within the pattern, we also tested an approximately M-scaled circular grating where the center spatial frequency $(4.4 \mathrm{cpd})$ was scaled across the radius, resulting in $0.16 \mathrm{cpd}$ at the stimulus perimeter. Three different stimulus conditions ( $L-M, L+M$, and $S$ ) were used that isolated the L-M cone opponent (or red-green), luminance, and the S-cone opponent (blue-yellow) pathways, respectively. Figure 2.18 illustrates the spatial layouts of the three pathwayisolating stimuli as well as different spatial frequency combinations used in the fMRI experiments. The stimuli had a mean luminance level of $127 \mathrm{~cd} / \mathrm{m}^{2}$ (equal to the luminance of the control background) and were adjusted to produce a mean cone-contrast of $29 \%$, calculated as the vector length (square root of the sum of squares) of individual cone contrasts (Cole et al., 1993). 
Materials and Methods

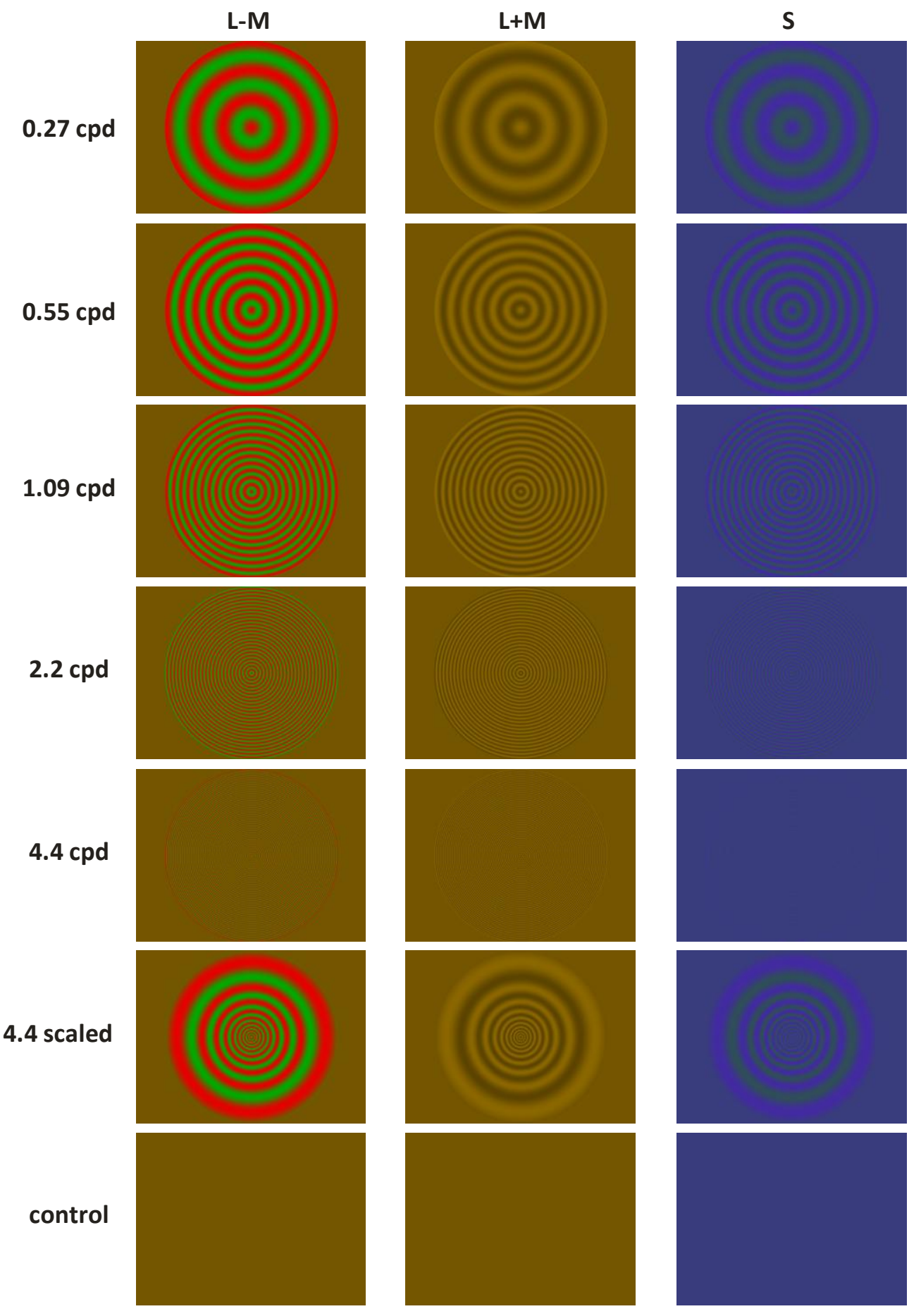

Figure 2.18: Spatial layouts of the pathway-selective stimuli at various spatial frequencies

Circular sine-wave gratings subtending a visual angle of 21 deg (diameter) were used. Stimuli to selectively stimulate the red-green, luminance, and blue-yellow pathways, respectively, are reproduced in three columns. Downsized versions for the various spatial frequencies $(0.27,0.55,1.09,2.2$, and $4.4 \mathrm{cpd})$ are shown in row 1 to 5 . Row 6 shows scaled stimuli. The latter have a center spatial frequency of $4.4 \mathrm{cpd}$ which is scaled across the radius, resulting in $0.16 \mathrm{cpd}$ at the stimulus perimeter. Corresponding control background stimuli are shown in the last row. Note that these printed images are for illustration only; they neither accurately represent the stimuli's actual chromaticity due to the different color-generating process, nor does the printer faithfully resolve these downsized images at the higher spatial frequencies. 


\subsubsection{Experimental design}

Each subject participated in three experimental runs; each run lasted ten minutes. In a typical run only one of the three stimulus types (e.g. luminance-pathway stimuli as shown in Figure 2.19) was shown for six different spatial frequencies $(0.27,0.55,1.09,2.2,4.4 \mathrm{cpd}$, and $4.4 \mathrm{cpd}$ scaled) using a block design paradigm (Henriksson et al., 2008).

Each experimental run commenced with a $24 \mathrm{~s}$ control period (data belonging to the first 12 seconds were discarded in the analysis), followed by six stimulus cycles. Each cycle (96 s) consisted of six stimulus blocks (12 s each) and a control block (24 s). In each stimulus block we presented grating stimuli with six different spatial frequency patterns. The order in which these spatial frequencies were presented was pseudo-randomized within each cycle and balanced across the run. During the control block, no stimulus appeared and a blank screen was shown. The latter had a chromaticity and luminance which was equal to the mean chromaticity and luminance of the grating stimulus. Each experimental run lasted 600 $s$ (300 volumes, $T R=2 \mathrm{~s})$. A small gray fixation cross was present throughout the experimental run.

To maintain attention and fixation during stimulus and control blocks, the volunteers performed a fixation task. The fixation cross was luminance modulated ( $2 \mathrm{~Hz}$ sinusoidal) with $50 \%$ Michelson contrast and at random intervals the contrast reduced to zero and a black cross appeared on the screen. In the fixation task, the subjects were asked to count the occurrences of the black fixation cross during the whole experimental run, in which there were 10-20 such events. All subjects performed well in the fixation task, detecting $>95 \%$ of the dimming events. 


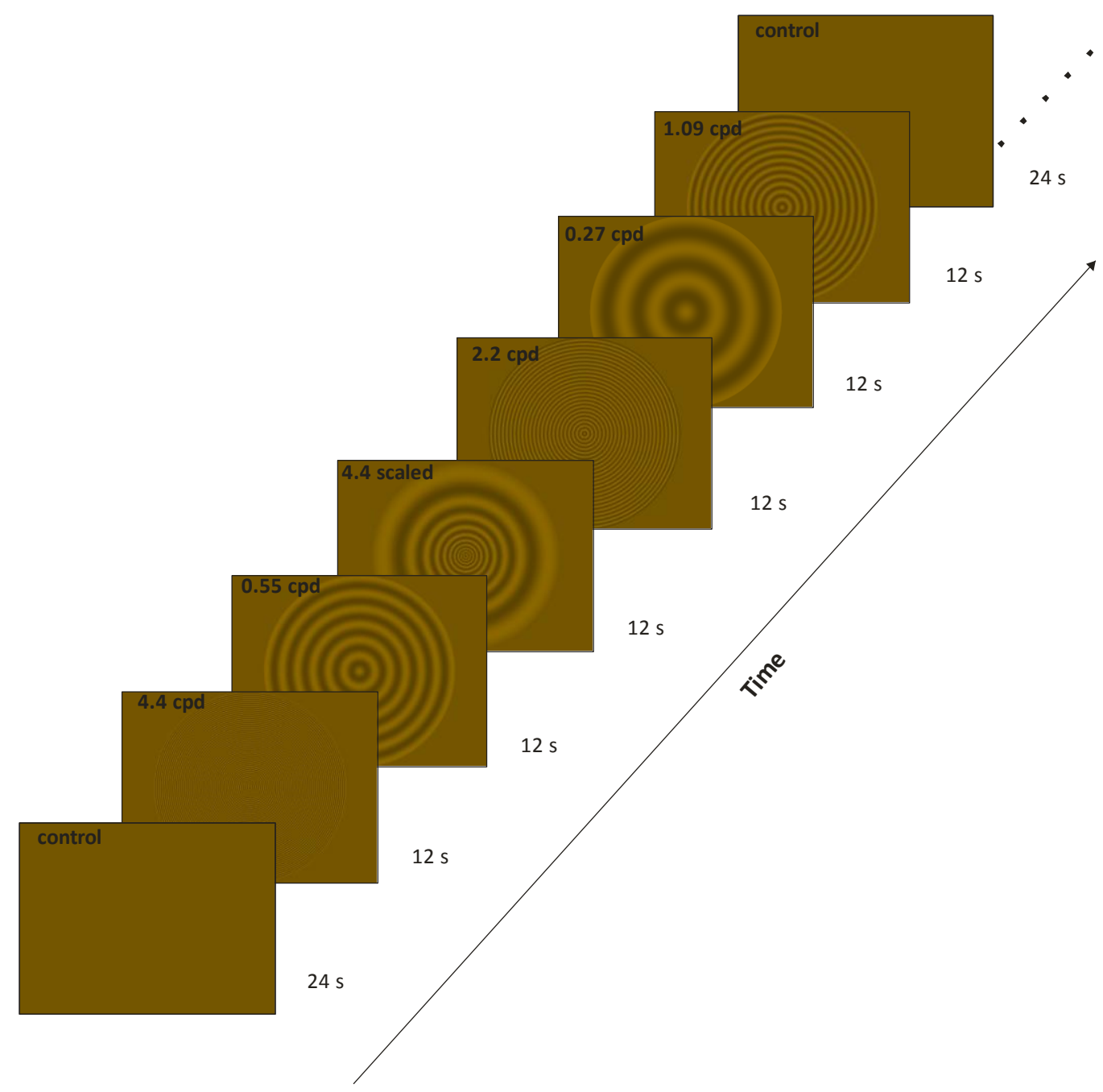

Figure 2.19: Schematic illustration of stimulus timing in the spatial frequency experiment

The above illustration is for stimulation of the luminance $(L+M)$ pathway. A central fixation cross was present at the center of the screen. Each experimental run commenced with a $24 \mathrm{~s}$ control period (data belonging to the first $12 \mathrm{~s}$ were discarded later in the analysis), followed by six stimulus cycles (only the first cycle is shown in the schematic). Each cycle (96 s) consisted of six stimulus blocks (12 s each) and a control block (24 s). In each stimulus block, the contrast of the grating stimulus was modulated at one of the six different spatial frequencies. The order in which the spatial frequencies were presented was pseudo-randomized within each cycle. 


\subsection{3 fMRI measurements and data processing}

All imaging studies were performed on a 2.9 T scanner (Magnetom Tim Trio, Siemens, Erlangen, Germany) using a 12-channel receive-only phased-array head coil.

Anatomical data: At the beginning of each spatial frequency experimental session (single day), T1-weighted 3D FLASH images $\left(1 \times 1 \times 1 \mathrm{~mm}^{3}\right)$ were acquired. The anatomical images served to align the functional data with the high-resolution structural images (i.e. MPRAGE data) acquired in the retinotopic session. The high-resolution T1-weighted 3D MPRAGE data set was used to reconstruct cortical surfaces for mapping and visualization purpose.

Functional data: A T2*-sensitive gradient echo-planar imaging (EPI) technique with an in-plane resolution of $2 \times 2 \mathrm{~mm}^{2}$ (repetition time (TR): $2000 \mathrm{~ms}$, echo time (TE): $36 \mathrm{~ms}$, flip angle: $70 \mathrm{deg}$, acquisition matrix: $96 \times 96$ ) was used to acquire functional volumes. Twentytwo consecutive sections of $4 \mathrm{~mm}$ thickness, approximately perpendicular to the calcarine fissure, covered early as well as the higher visual areas in the occipital lobe.

Data processing: Data analysis was performed using BrainVoyager QX 1.10 (Brain Innovation, Maastricht, The Netherlands). Preprocessing of the functional data included deletion of the initial 12 volumes (to allow longitudinal magnetization to reach a steady state), 3D-motion correction (also including intra-session alignment), slice-time correction, temporal high-pass filtering ( 3 cycles/run), linear trend removal, spatial smoothing with a Gaussian kernel (full width at half maximum $4 \times 4 \times 4 \mathrm{~mm}^{3}$ ). After preprocessing, functional data were co-registered to the anatomical volume (FLASH) acquired at the beginning of the same session, and subsequently transformed into Talairach space (Talairach \& Tournaux, 1988). 


\subsubsection{Eccentricity-based region-of-interest analysis}

The aim of the experiment described in this section was to identify dependence of the fMRI responses to stimulation of the chromatic and luminance channels, using grating stimuli of various spatial frequency patterns, and on visual field eccentricity. We therefore defined ROIs corresponding to the near-fovea and to increasing visual field eccentricities $(1.4,2.2$, $3.9,3.8,4.6,6.3,8.1$, and $9.8 \mathrm{deg}$ ) in the primary visual cortex (V1) and ventral visual areas (V2v, VP, and V4). ROIs (mean size: 28 voxels) were defined on the reconstructed cortical surface based on the meridian and eccentricity information available in the retinotopic mapping experiments. To estimate the strength of the fMRI response within the retinotopically mapped visual eccentricities, we performed a general linear model (GLM) analysis separately on each ROI. To do so, we first extracted the mean time course of a given ROI by averaging over all of its voxels. In the subsequent step, the time course was normalized using the z-transform. Next, the GLM was fit to the normalized time course with six predictors (corresponding to the six spatial-frequency patterns) and a baseline (control block). The time course of the predictors was computed from the stimulation protocol and was convolved with the two-gamma hemodynamic response function. As shown in the GLM equation below, each predictor time course is associated with a coefficient or beta weight 6 , quantifying its potential contribution in explaining the ROI time course $y$. The beta weights in this context reflect the strength of the fMRI signal in response to stimulation at different spatial frequencies $(0.27,0.55,1.09,2.2,4.4 \mathrm{cpd}$, and $4 \mathrm{cpd}$ scaled).

$$
y=b_{\text {baseline }}+b_{0.27} x_{1}+b_{0.55} x_{2}+b_{1.09} x_{3}+b_{2.2} x_{4}+b_{4.4} x_{5}+b_{4.4 s c a l e d} x_{6}+\varepsilon
$$




\subsubsection{Spatial frequency tuning curves}

To characterize the spatial-frequency tuning properties at a given eccentricity, we fitted Gaussian functions to the data. The four-parameter Gaussian function used in the analysis is given by

$$
R=R_{0}+R_{1} \exp \left(-(f-\mu) / 2 \sigma^{2}\right),
$$

where $R$ is the fMRI response (beta-weight) at a given spatial frequency $f$, and $R_{0}, R_{1}, \mu$, are parameters that were estimated using the Marquardt-Levenberg algorithm in SigmaPlot . The tuning curves were fitted to data averaged across six hemispheres (three subjects). As described by Henriksson et al. (2008), for each data with band-pass tuning characteristics, the fitted parameter $\mu$ indicated the optimal spatial frequency. And for the tuning data with low-pass or high-pass tuned characteristics, the lowest or the highest spatial frequency correspondingly was defined as the optimum. 


\subsection{Temporal frequency experiments}

\subsubsection{Visual stimuli}

In the second set of experiments designed to study the influence of stimulus temporal frequency on activation in visual areas, the stimuli consisted of contrast-reversing circular sine-wave gratings, spanning a visual angle of 21 deg (diameter) with the center cross (1 deg in diameter) used for the fixation task. To minimize the influence of cortical magnification, we only used M-scaled stimuli; they had a spatial frequency of $4 \mathrm{cpd}$ at the center and were scaled such that the spatial frequency was $0.16 \mathrm{cpd}$ at the stimulus perimeter. Three different stimuli (L-M, L+M, and S) were used that isolated the L-M cone opponent (or redgreen), the luminance, and the S-cone opponent (blue-yellow) pathways, respectively. Figure 2.20 illustrates the spatial layout of the three pathway-isolating stimuli used in the $\mathrm{fMRI}$ experiments. The stimuli had a mean luminance of $127 \mathrm{~cd} / \mathrm{m}^{2}$ (equal to the luminance of the control stimulus), and produced a mean cone-contrast of $29 \%$, calculated as the vector length (square root of the sum of squares) of individual cone-contrasts.
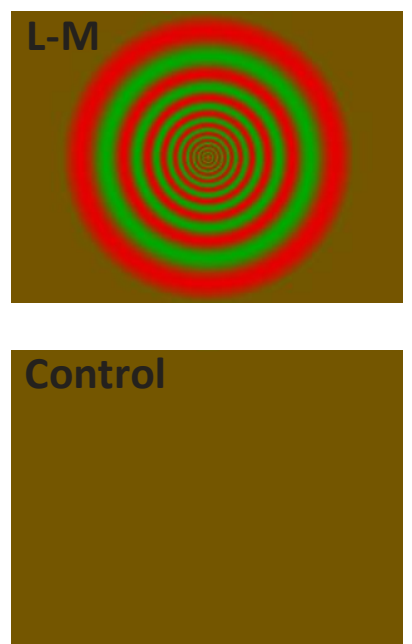
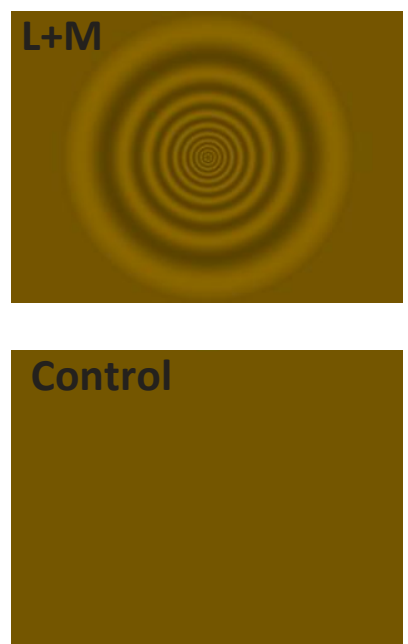
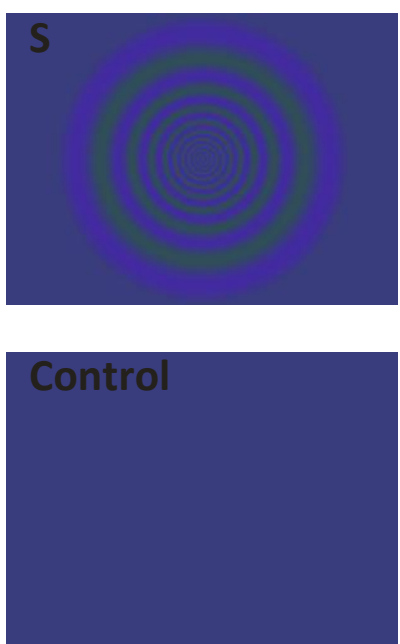

Figure 2.20: Spatial layouts of the pathway-selective stimuli

Circular sine-wave gratings subtending a visual angle of 21 deg (diameter) with spatial frequency scaled to compensate for cortical magnification. Stimuli to selectively stimulate the red-green $(L-M)$, luminance $(L+M)$, and blue-yellow (S) pathways, respectively, are shown in three columns (first row), corresponding control stimuli are shown in the second row. Note that the printed images are not an accurate representation of the actual stimuli. 


\subsubsection{Experimental design}

Each subject participated in six experimental runs, each of which lasted ten minutes. In a typical run only one of the three stimulus conditions (e.g. L-M stimuli in Figure 2.21) was shown at six different sinusoidal modulation frequencies $(2,4,6,8,10$, and $12 \mathrm{~Hz})$ using a block design paradigm. Two runs were acquired per stimulus type.

A similar experimental design was used to that in the spatial frequency experiments. Briefly, each experimental run commenced with the $24 \mathrm{~s}$ control period followed by six stimulus cycles. Again, each cycle (96 s) consisted of six stimulus blocks (12 s each) and a control block (24 s). In each stimulus block, the contrast of the grating stimulus was modulated at one of the six different temporal frequencies. As before, the order in which the temporal frequencies were presented was pseudo-randomized within each cycle and balanced across the run. During the control block, no stimulus appeared and a blank screen was shown. Each experimental run lasted $600 \mathrm{~s}$ (300 volumes, TR $=2 \mathrm{~s}$ ). The small gray fixation cross was present throughout the experimental run. 


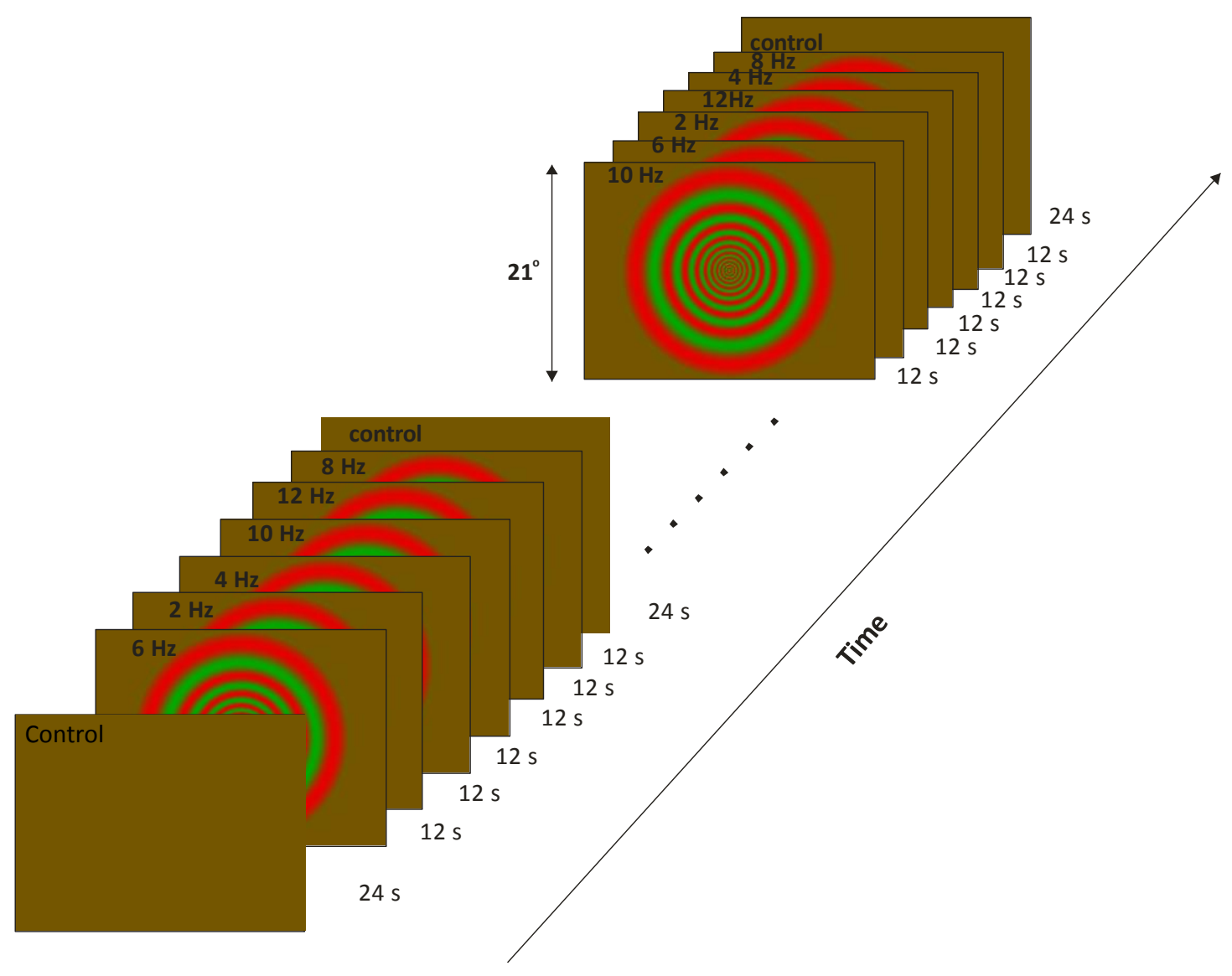

Figure 2.21: Schematic illustration of stimulus timing

The above illustration is for stimulation of the L-M (or red-green) pathway. A central fixation cross was present at the center of the screen. Each experimental run commenced with a $24 \mathrm{~s}$ control period (data belonging to the first 12 seconds were discarded later in the analysis) followed by six stimulus cycles. Each cycle (96 s) consisted of six stimulus blocks (12 s each) and a control block (24 s). In each stimulus block, the contrast of the grating stimulus was modulated at one of the six different temporal frequencies. The order in which the temporal frequencies were presented was pseudo-randomized within each cycle. 


\subsection{3 fMRI measurements and data processing}

fMRI measurements and data processing steps were similar to those described in section 2.7.3.

\subsubsection{Visual area based region-of-interest analysis}

To estimate the strength of the $\mathrm{fMRI}$ response within the retinotopically mapped visual areas, we performed a general linear model (GLM) analysis separately on each ROI. To do so, we first extracted the mean time course of a given ROI by averaging over all voxels. In the subsequent step, the time course was normalized using the z-transform Next, the GLM was fit to the normalized time course with six predictors (corresponding to six temporal frequencies) and a baseline (control block). The time course of the predictors was computed from the stimulation protocol and was convolved with the two-gamma haemodynamic response function As shown in GLM equation below, each predictor time course is associated with a coefficient or beta weight $b$, quantifying its potential contribution in explaining the ROI time course $y$. The beta weights in this context reflect the strength of the $\mathrm{fMRI}$ signal in response to stimulation at different temporal frequencies $(2,4,6,8,10$, and $12 \mathrm{~Hz})$.

$$
y=B_{\text {baseline }}+b_{2} x_{1}+b_{4} x_{2}+b_{6} x_{3}+b_{8} x_{4}+b_{10} x_{5}+b_{12} x_{6}+\varepsilon
$$

\subsubsection{Temporal frequency tuning curves}

For each visual area under investigation, the $\mathrm{fMRI}$ response (beta-weight) was plotted as a function of temporal frequency of the grating stimuli for three stimulus conditions: L-M (or red-green), L+M (or luminance), and S (blue-yellow), respectively. Because tuning curves can often be summarized by a Bode plot, approximating the response function by piecewise linear functions of log temporal frequency, we attempted a similar description of the fMRI response data. From visual inspection of the plots, a piece-wise linear fit with a break at $4 \mathrm{~Hz}$ 
would seem to fit the data well, so that the sections between 2 and $4 \mathrm{~Hz}$ and 4 and $12 \mathrm{~Hz}$ were fitted by two first-order polynomials (straight lines)

$$
R=s f+c
$$

to the data, where $R$ is the fMRI response at a particular temporal frequency, $s$ is the slope of the tuning curve, $f$ is the temporal frequency and $c$ is a constant. The sign and the slopes of the fitted lines indicate whether there is any systematic increase or decrease in response with ( $\log$ ) frequency. We also determined $p$ values of the slopes, which indicated the significance of the inclination or declination of the slope. An example for straight line fitting is provided in Figure 2.22.

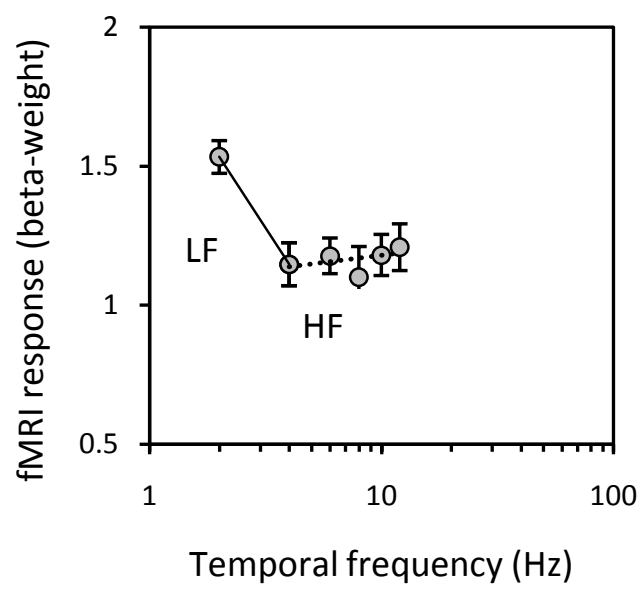

Figure 2.22: Example of a temporal frequency tuning curve

The fMRI response is plotted as a function of temporal frequency. As can be seen in the plot, there are two linear trends present in the data. The LF (low frequency) branch represents a straight line between 2 and $4 \mathrm{~Hz}$, and the HF (high frequency) branch represents the data between 4 and $12 \mathrm{~Hz}$.

\subsubsection{Cluster analysis of visual areas}

A hierarchical cluster analysis was performed to investigate whether the visual areas could be grouped into clusters based on their similarity in processing of temporal-frequency related chromatic and luminance information. For this purpose, we used the slope values $\left(\mathrm{S}_{\mathrm{LF}}\right.$ and $\left.\mathrm{S}_{\mathrm{HF}}\right)$ of the fitted low-frequency and high-frequency curves as input to the cluster analysis algorithm. In total there were 48 slope values (eight visual areas: MT, V3a, V3d, 
V2d, V1, V2V, VP, and V4; three stimulus conditions: L-M, L+M, and S; two slopes: $S_{L F}$ and $\mathrm{S}_{\mathrm{HF}}$ ). Cluster analysis was performed using the SPSS 16.0 statistical package. We used the default between-group linkage procedure for combining clusters and the Squared Euclidian distance for the distance measure. The output of the hierarchical cluster analysis is represented as a dendrogram. 


\section{3}

Results 


\subsection{Dependence of chromatic and luminance responses on visual field eccentricity and spatial frequency}

The aim was to investigate any dependence of the BOLD fMRI responses to the chromatic and luminance stimuli on visual field eccentricity. $\mathrm{FMRI}$ responses to selective stimulation of the chromatic (red-green or L-M cone-opponent; blue-yellow or S-cone) and luminance $(L+M)$ pathways were measured in ROls corresponding to the near-fovea ( $1.5 \mathrm{deg})$, and at higher visual field eccentricities up to approximately $10 \mathrm{deg}$, in retinotopically mapped visual areas (V1, V2V, VP, and V4). It is well established that there is a steeper loss of redgreen than luminance contrast sensitivity across the visual field psychophysically (Mullen, 1991; Stromeyer et al., 1992; Mullen \& Kingdom, 2002; Mullen et al., 2005). It has previously been reported that responses in the primary visual cortex (V1) to red-green stimulation are stronger close to the fovea and decline across eccentricity (Vanni et al., 2006; Mullen et al., 2007) for stimuli with relatively low spatial frequency. The effect of spatial frequency on eccentricity-dependent $f M R I$ responses was investigated by measuring responses to circular sine-wave gratings at a range of spatial frequencies $(0.27,0.55,1.09$, 2.2 , and $4.4 \mathrm{cpd}$ ). In addition to using grating stimuli with a fixed spatial frequency within the pattern, also M-scaled circular gratings, where the spatial frequency increases across the radius according to the cortical magnification factor, were tested.

\subsubsection{Sensitivity as a function of visual field eccentricity in V1}

In Figure 3.1, fMRI responses in V1 averaged across six hemispheres are plotted as a function of visual field eccentricity as extracted from our retinotopic results, for a set of spatial frequencies. The data show that V1 responds robustly to red-green as compared to luminance and blue-yellow stimulus conditions across all spatial frequencies. This result is in line with previous fMRI reports on cortical processing of chromatic modulation (Kleinschmidt et al., 1996; Engel et al., 1997a; Liu \& Wandell, 2005; Wade et al., 2008). For a spatial frequency $0.27 \mathrm{cpd}$ (Figure 3.1a), we found that both red-green and luminance responses increase as a function of eccentricity with peaks at about 8 deg eccentricity and a drop thereafter. Blue-yellow responses, however, showed only a minor increase across 
eccentricity. A similar response pattern can be seen at $0.55 \mathrm{cpd}$ (Figure 3.1b) with the peak slightly shifted to the left. Similarly, we observe that the peak response shifts progressively towards lower eccentricities as the spatial frequency of the stimulus increases further (Figure 3.1c-e) indicating the expected foveal specialization for higher spatial frequencies. At spatial frequencies 2.2 and $4.4 \mathrm{cpd}$, functional responses decline steeply with increasing eccentricity. This is consistent with receptive fields getting larger with increasing eccentricity and thus making a high spatial frequency stimulus less effective.

Finally, we tested whether an M-scaled grating stimulus results in evenly distributed responses across eccentricities (Figure 3.1f). A center spatial frequency of $4.4 \mathrm{cpd}$, which was scaled across the radius, resulting in $0.16 \mathrm{cpd}$ at the stimulus perimeter for red-green, luminance, and the blue-yellow stimuli, was used. For red-green the data show that the scaling was effective in bringing-about the largest responses overall and a reasonably flat characteristic except for the largest eccentricity $(9.8 \mathrm{deg})$ where a still lower spatial frequency might have yielded larger responses. The same can be said about the luminance stimulus. For the blue-yellow stimulus, the largest responses were obtained at $4.6 \mathrm{deg}$, after which there was a pronounced linear decline with log spatial frequency, indicating that a different scaling of spatial frequency may be necessary in order to achieve an even distribution of responses across eccentricity.

In the following section the optimum spatial frequencies at different eccentricities for red-green, luminance and blue-yellow stimulus conditions, respectively, were estimated. We estimate new scaling values for the M-scaled stimulus, for each condition, to achieve an even distribution of responses across eccentricities. 


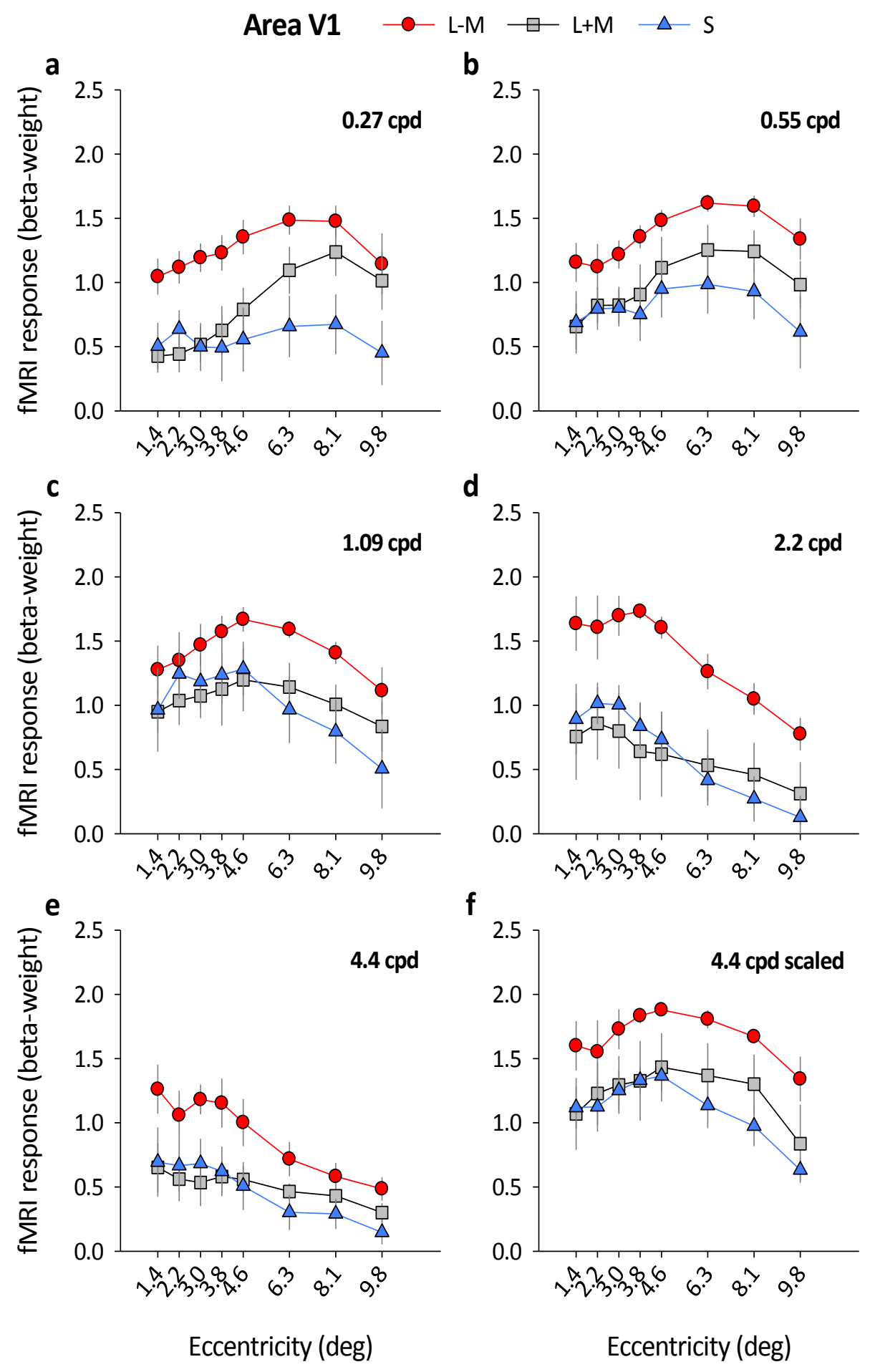

Figure 3.1: Sensitivity in V1 as a function of visual field eccentricity

fMRI responses averaged over six hemispheres, plotted as a function of visual field eccentricity for stimulation of the three post-receptoral pathways: L-M, L+M, and $S$, respectively. The subplots show eccentricitydependent responses at stimulus spatial frequencies of a) $0.27 \mathrm{cpd}$, b) $0.55 \mathrm{cpd}, \mathrm{c}) 1.09 \mathrm{cpd}, \mathrm{d}) 2.2 \mathrm{cpd}$, and e) $4.4 \mathrm{cpd}$, respectively. f) $4.4 \mathrm{cpd}$ scaled - here the stimulus had a central spatial frequency of $4.4 \mathrm{cpd}$, which was scaled along the radius by an estimate of the cortical magnification function, resulting in a spatial frequency of $0.16 \mathrm{cpd}$ at the periphery. The data are color coded according to stimulus conditions; error bars indicate \pm s.e.m. The data show that relative responses depend, across eccentricity, on stimulus spatial frequency. 


\subsubsection{Sensitivity as a function of spatial frequency across visual field eccentricity in V1}

As indicated in the previous section, the eccentricity-dependent responses in area V1 are affected by the spatial frequency of the stimulus. High spatial frequencies elicit stronger responses at the central parts of the visual field, whereas low spatial frequencies are favored by the peripheral parts of the visual field. In Figure 3.2, we estimate the optimum spatial frequency from tuning functions at the three tested eccentricities (circles: 1.4 deg; squares: $4.6 \mathrm{deg}$; triangles: $9.8 \mathrm{deg}$ ) in response to stimulation of the red-green, luminance, and blue-yellow pathways, respectively. Spatial frequencies were distributed between 0.27 and $4.4 \mathrm{cpd}$.

To characterize the spatial-frequency tuning properties at a given eccentricity, we fitted Gaussian functions to the data, following the fitting procedure explained in Henriksson et al. (2008). For red-green (Figure 3.2a) and luminance stimulus conditions (Figure 3.2c), the tuning curves are band-pass for all characterized eccentricities, with the peak spatial frequency declining with increasing visual field eccentricity. Similar findings have been reported in a recent study (Henriksson et al., 2008), using achromatic 'sinring' (Mullen et al., 2005) grating patterns. For the blue-yellow stimulus (Figure 3.2e), the tuning curves are also band-pass; however, their peak spatial frequencies lie close to each other. Optimum spatial frequencies in V1 across different eccentricities for different stimulus conditions are summarized in Table 3.1. We note that in the periphery (e.g. at 9.8 deg) low spatial frequency stimuli are required to obtain responses equivalent to those at the center (e.g. $1.4 \mathrm{deg})$. This is reflected in the sequential leftwards shift of the tuning functions as eccentricity increases.

The eccentricity dependence can be accounted for by applying scaling factors to the peripheral curves to superimpose them upon the 1.4 deg tuning function (Watson, 1987; Vakrou et al., 2005). This is shown in Figure 3.2, right column, for the red-green, luminance and blue-yellow stimuli, respectively. In the plots, the data represent the spatial frequency scaling values across eccentricity, normalized with respect to the peak spatial frequency of the 1.4 deg tuning function, i.e. peak spatial frequencies of the 4.6 and 9.8 deg tuning functions are divided by the 1.4 deg peak spatial frequency. 

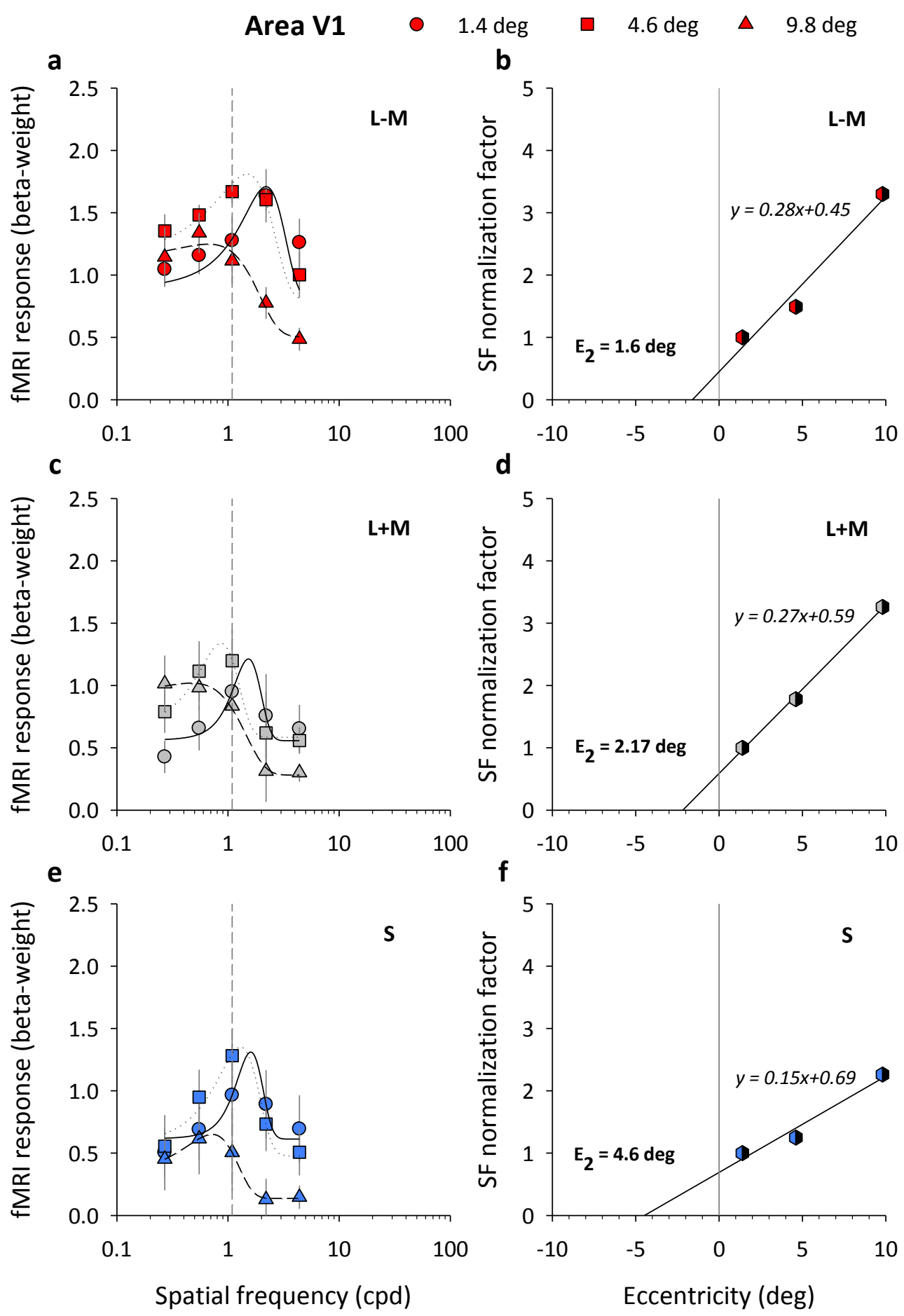

Figure 3.2: Spatial frequency tuning functions in V1 across different eccentricities

Left column: fMRI responses averaged over six hemispheres plotted as a function of spatial frequency across different eccentricities (o: $1.4 \mathrm{deg}$; $\square: 4.6 \mathrm{deg} ; \Delta: 9.8 \mathrm{deg}$ ) for stimulation of the three post-receptoral pathways: a) L-M, c) L+M, and e) S, respectively. For visualization purposes and for quantification of the optimum spatial frequencies, Gaussian functions (solid line: 1.4 deg; dotted line: $4.6 \mathrm{deg}$; short dashed line: 9.8 $\mathrm{deg}$ ) are fitted to the data. The vertical short dashed line represents the mean along the spatial frequency axis and serves as a reference. Right column: Spatial frequency scaling factors required for normalizing the three scaling functions at eccentricities $1.4,4.6$, and 9.8 deg to that at 1.4 deg by matching their optimum spatial frequency values. Straight lines are fitted to the data. $E_{2}$ value is the eccentricity axis intercept. 
Next we fitted linear regression lines to characterize the linear relationship between the visual field eccentricity and spatial-frequency normalization factor. The linear relationship between eccentricity and spatial frequency normalization factor can be summarized by the horizontal intercept $-\mathrm{E}_{2}$ (Levi et al., 1984; Levi et al., 1985) and slope of the fitted straight line (Slotnick et al., 2001). A smaller $E_{2}$ corresponds to a steeper scaling function, i.e. lower spatial frequency is required at larger eccentricity to match the performance at 1.4 deg. $E_{2}$ values are displayed on each plot corresponding to the three post-receptor channels. We found different scaling functions for each of the three mechanisms. For the red-green mechanism, the $E_{2}$ value was $1.6 \mathrm{deg}$. For the luminance mechanism, $E_{2}$ was 2.17 deg. Finally, for the blue-yellow mechanism we found an $E_{2}$ value of $4.6 \mathrm{deg}$.

\begin{tabular}{lccc}
\hline \hline Stimulus & $1.4 \mathrm{deg}$ & $4.6 \mathrm{deg}$ & $9.8 \mathrm{deg}$ \\
\hline $\mathrm{L}-\mathrm{M}$ & 2.19 & 1.48 & 0.67 \\
\hline $\mathrm{L}+\mathrm{M}$ & 1.53 & 0.86 & 0.47 \\
$\mathrm{~S}$ & 1.6 & 1.29 & 0.72 \\
\hline
\end{tabular}

Table 3.1: Optimum spatial frequencies (in cpd) across eccentricity in V1 for stimulation of the three postreceptoral pathways

The optimum spatial frequencies, i.e. the peak spatial frequencies of the tuning functions were obtained by fitting Gaussian functions to the mean (of six hemispheres) tuning data. 


\subsubsection{Sensitivity as a function of visual field eccentricity in extrastriate visual areas (V2v, VP, and V4)}

Following the same approach as described in Section 3.1.1 for V1, the visual field eccentricity-dependent responses are described in this section for ventral visual areas $V 2 v$, VP, and V4, respectively, again for stimulation of the red-green, luminance and blue-yellow mechanisms using a set of spatial frequencies.

Area V2v: In Figure 3.3, fMRI responses in V2v averaged across six hemispheres are plotted as a function of visual field eccentricity for a set of spatial frequencies. The data show that V2v exhibits a quite similar response profile to V1. It was noticed that for lower spatial frequencies responses increase as a function of eccentricity, and for high spatial frequencies responses decline with increasing eccentricity. It is of note that the peak response is shifted to a lower eccentricity at $1 \mathrm{cpd}$, as compared to $\mathrm{V} 1$, reflecting that receptive fields are larger in V2v relative to V1. For the scaled stimulus (center frequency 4.4 $\mathrm{cpd}, \mathrm{SF}$ at the perimeter $0.16 \mathrm{cpd}$ ), we see the best overall response profile (3.3f). Figure 3.4 illustrates the spatial frequency tuning properties in V2v across different eccentricities. The $E_{2}$ values, obtained as before by normalizing the tuning curves, are shown in the plots.

Area VP and V4: In Figure 3.5 and Figure 3.6, fMRI responses in VP and V4, respectively, averaged across six hemispheres, are plotted as a function of visual field eccentricity for various spatial frequencies. Both in VP (except for $0.27 \mathrm{cpd}$ ) and V4, we did not see an increase in response across eccentricity for low spatial frequencies but mostly rather a decline with eccentricity. One plausible explanation to this could be that in VP and V4, the receptive fields get relatively larger at lower eccentricities, so that much lower spatial frequencies (higher scaling values) are required to produce equivalent responses as compared to $1.4 \mathrm{deg}$. This is apparent in Figure $3.6 \mathrm{f}$ for the red-green response, where the high spatial frequencies at the lower most eccentricities evoke stronger responses; however, despite scaling of spatial frequency, the stimulus failed to evoke equally strong responses across eccentricities. Insofar as V4 has been considered to play an essential role in processing chromatic information, this may imply that loss of red-green chromatic sensitivity in peripheral vision occurs at this locus. 


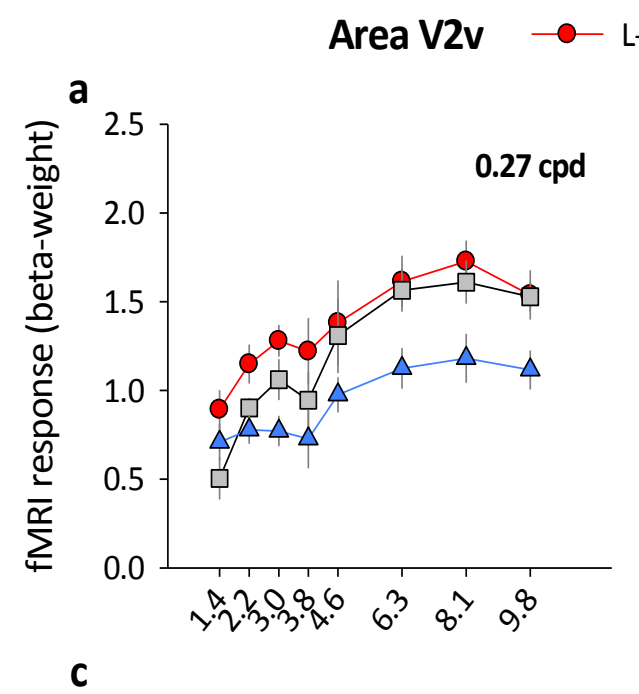

$-M \square L+M \backsim S$

b
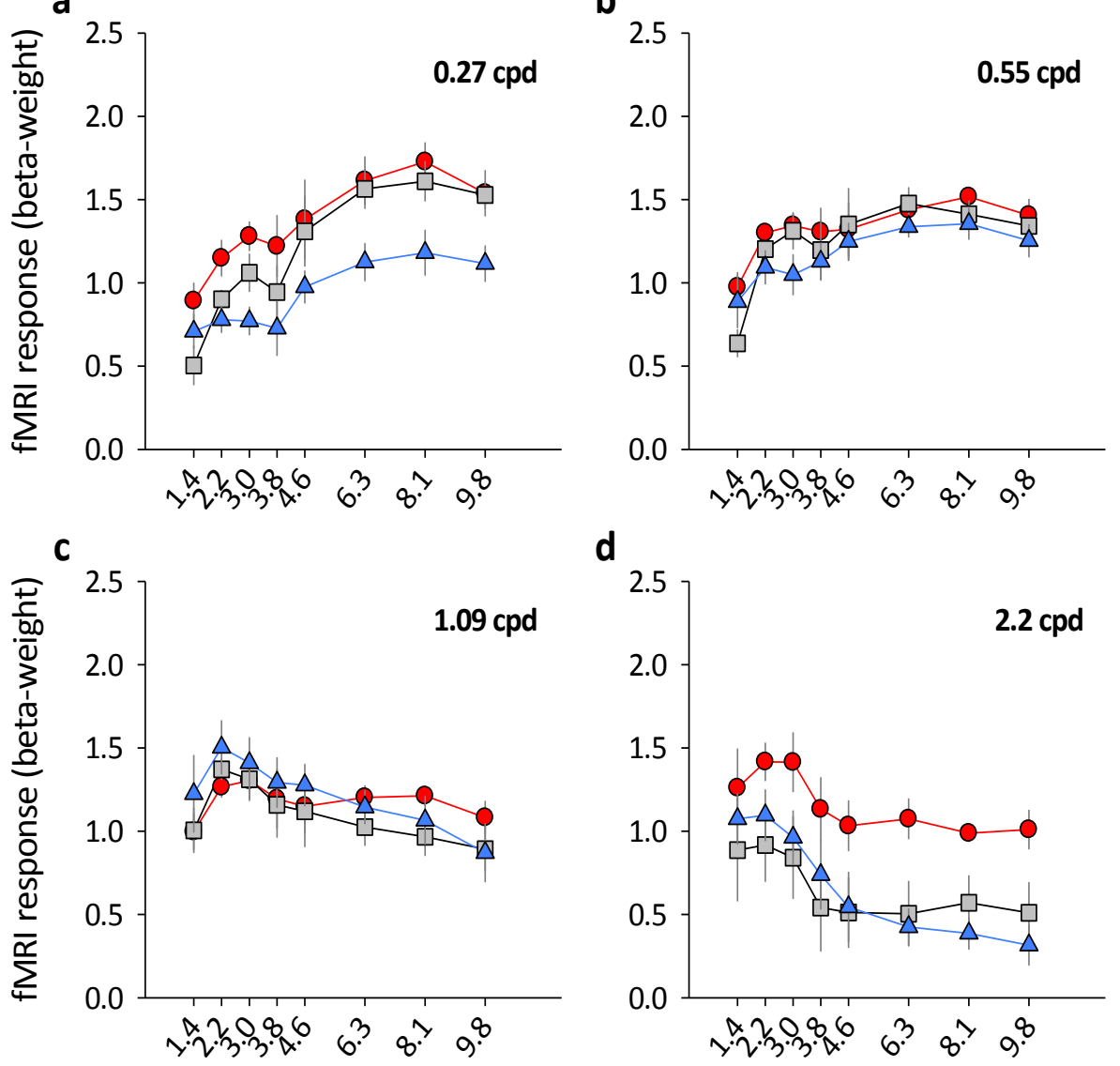

d
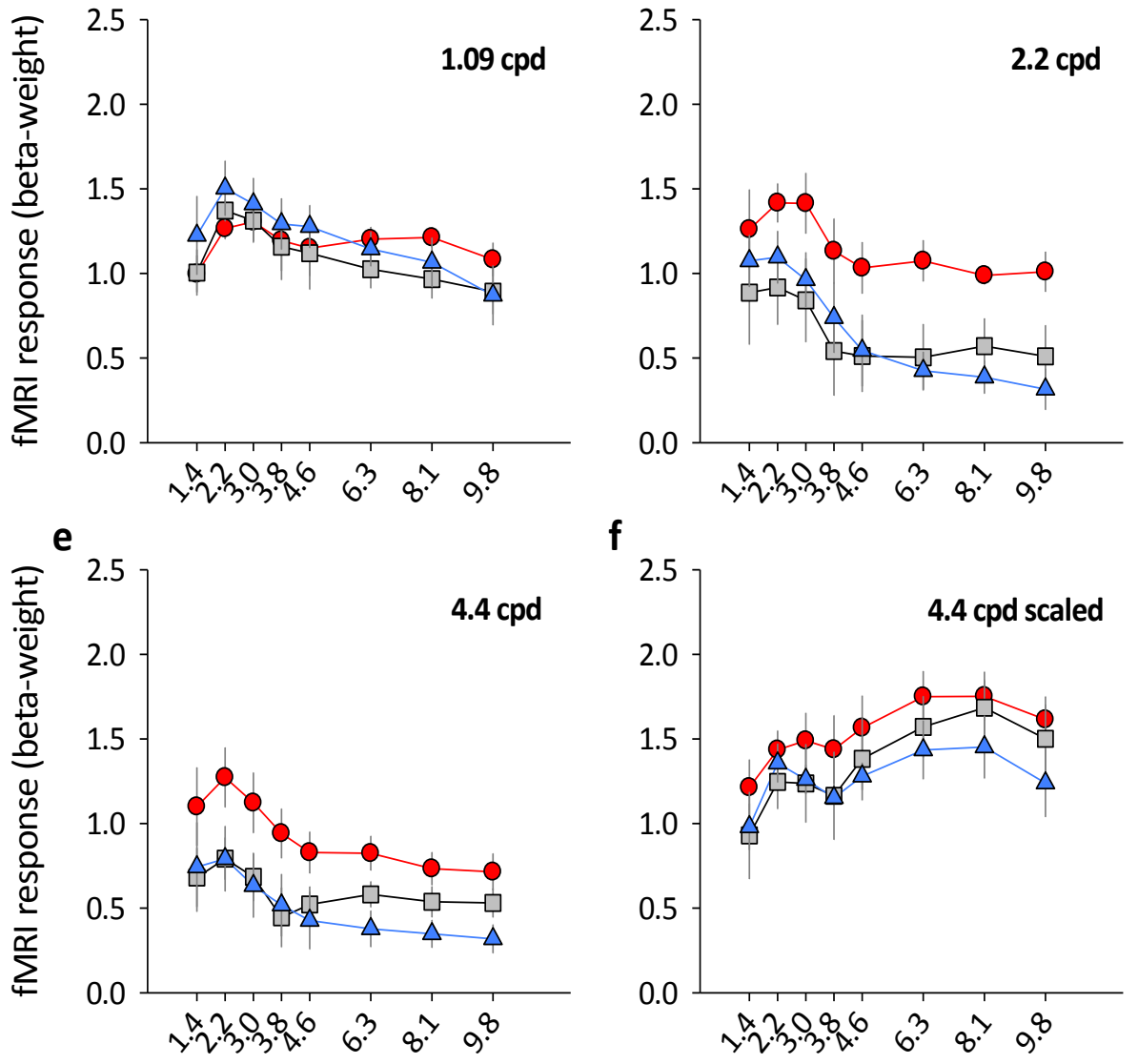

f

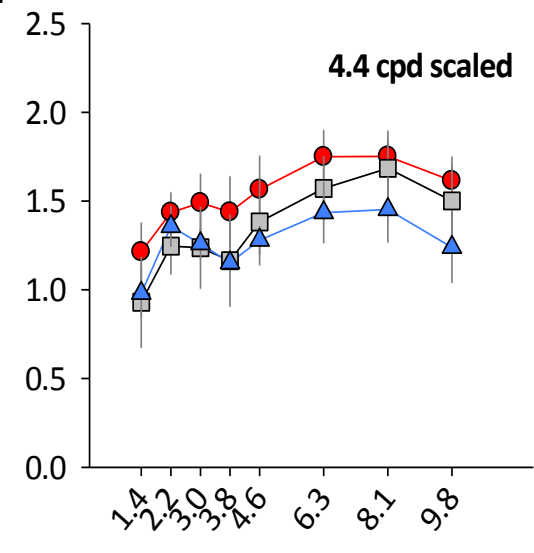

Eccentricity (deg)

Eccentricity (deg)

Figure 3.3: Sensitivity in V2v as a function of visual field eccentricity

fMRI responses averaged over six hemispheres plotted as a function of visual field eccentricity for stimulation of the three post-receptoral pathways: L-M, L+M, and $S$, respectively. The subplots show eccentricitydependent responses at stimulus spatial frequencies of a) $0.27 \mathrm{cpd}$, b) $0.55 \mathrm{cpd}, \mathrm{c}) 1.09 \mathrm{cpd}, \mathrm{d}) 2.2 \mathrm{cpd}$, and e) $4.4 \mathrm{cpd}$, respectively. f) The stimulus comprised a central spatial frequency of $4.4 \mathrm{cpd}$, which is scaled along the visual field eccentricities by an estimate of the cortical magnification function, resulting in a spatial frequency of $0.16 \mathrm{cpd}$ at the periphery.. The data are color coded according to stimulus conditions. Error bars indicate \pm s.e.m. The data show that relative responses to the different stimuli across eccentricity depend on spatial frequency of the stimulus. 

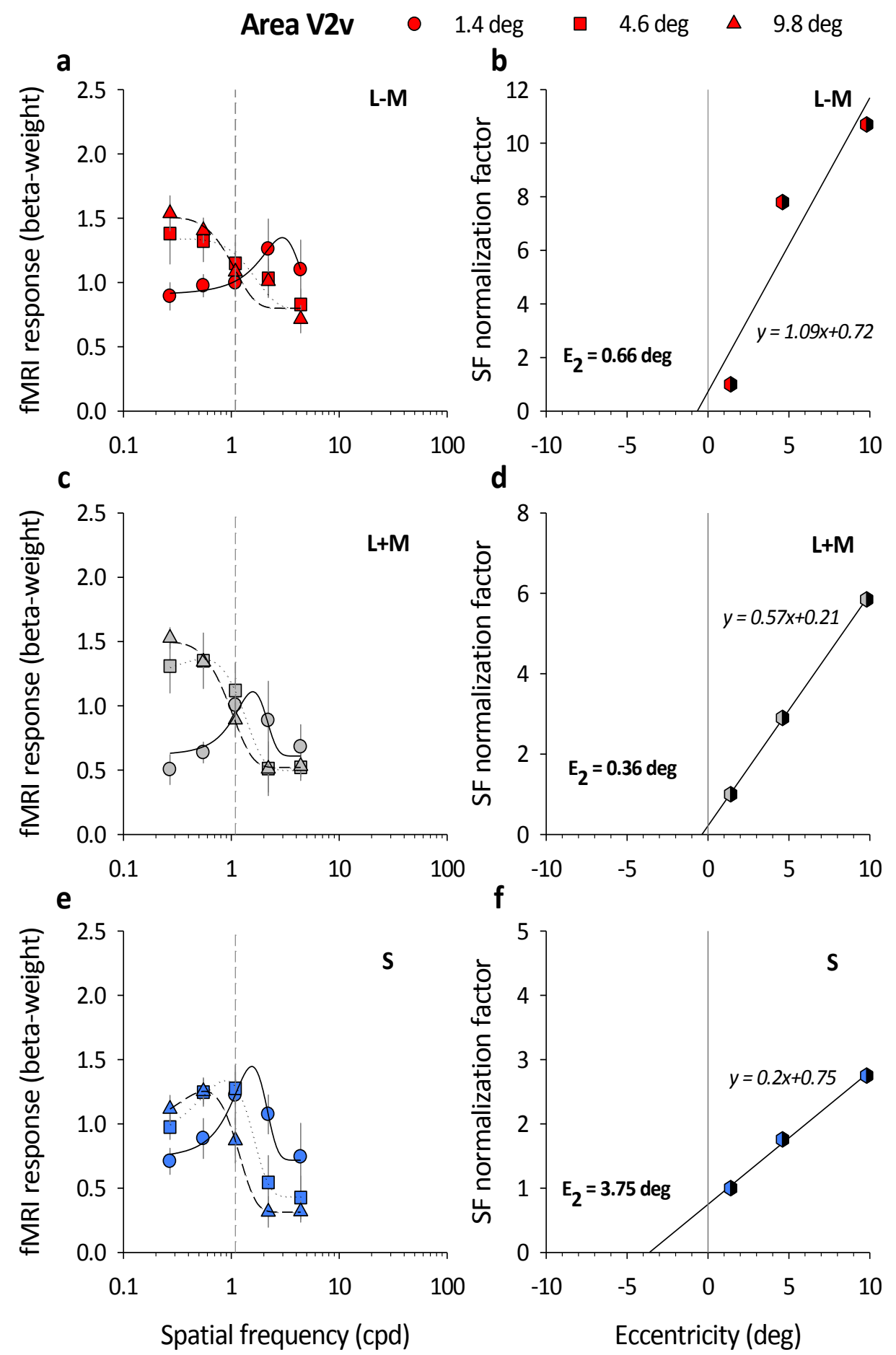

Figure 3.4: Spatial frequency tuning functions in V2v across different eccentricities

fMRI responses averaged over six hemispheres plotted as a function of spatial frequency across different eccentricities (o: $1.4 \mathrm{deg}$; $\square: 4.6 \mathrm{deg} ; \Delta: 9.8 \mathrm{deg}$ ) for stimulation of the three post-receptoral pathways: a) L-M, c) $\mathrm{L}+\mathrm{M}$, and e) $\mathrm{S}$, respectively. For visualization purposes and for quantification of the optimum spatial frequencies, Gaussian functions (solid line: $1.4 \mathrm{deg}$; dotted line: $4.6 \mathrm{deg}$; dashed line: $9.8 \mathrm{deg}$ ) are fitted to the data. The vertical short dashed line represents the mean along the spatial frequency axis and serves as a reference. b, d, \& f) Optimum spatial frequency values at eccentricities 1.4, 4.6, and 9.8 deg, respectively, are normalized with respect to the 1.4 deg optimum spatial frequency value. Straight lines are fitted to the data. $E_{2}$ value is the eccentricity axis intercept. 


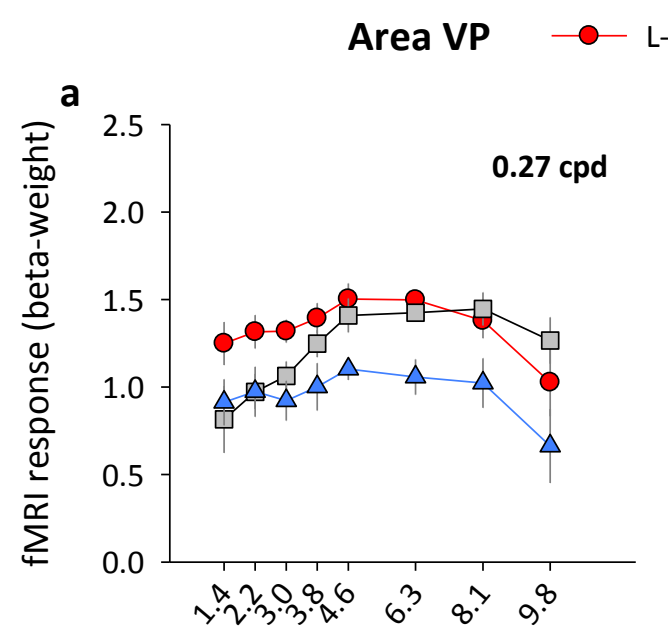

$-\mathrm{M} \square \mathrm{L}+\mathrm{M} \longrightarrow \mathrm{S}$

b
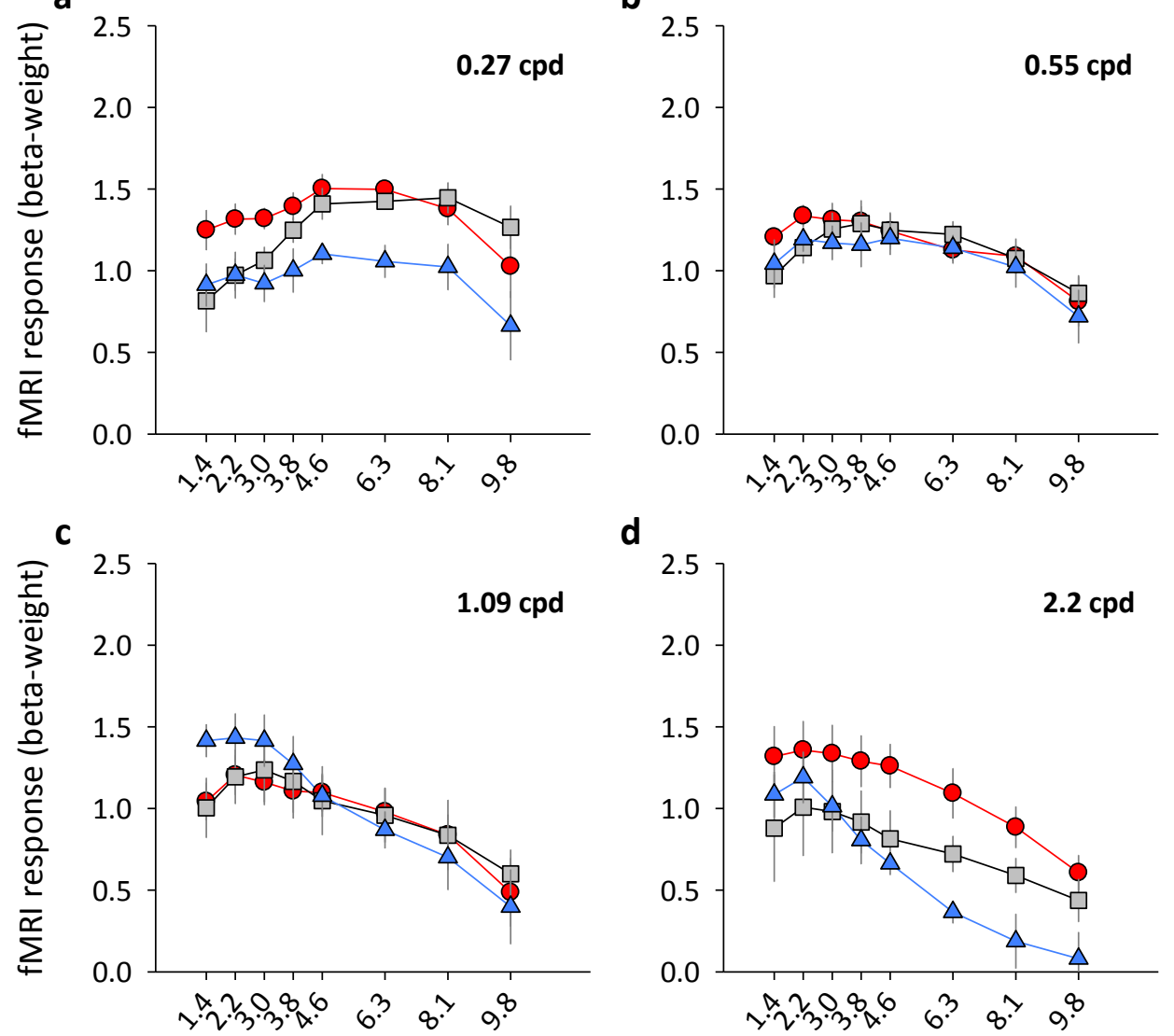

d
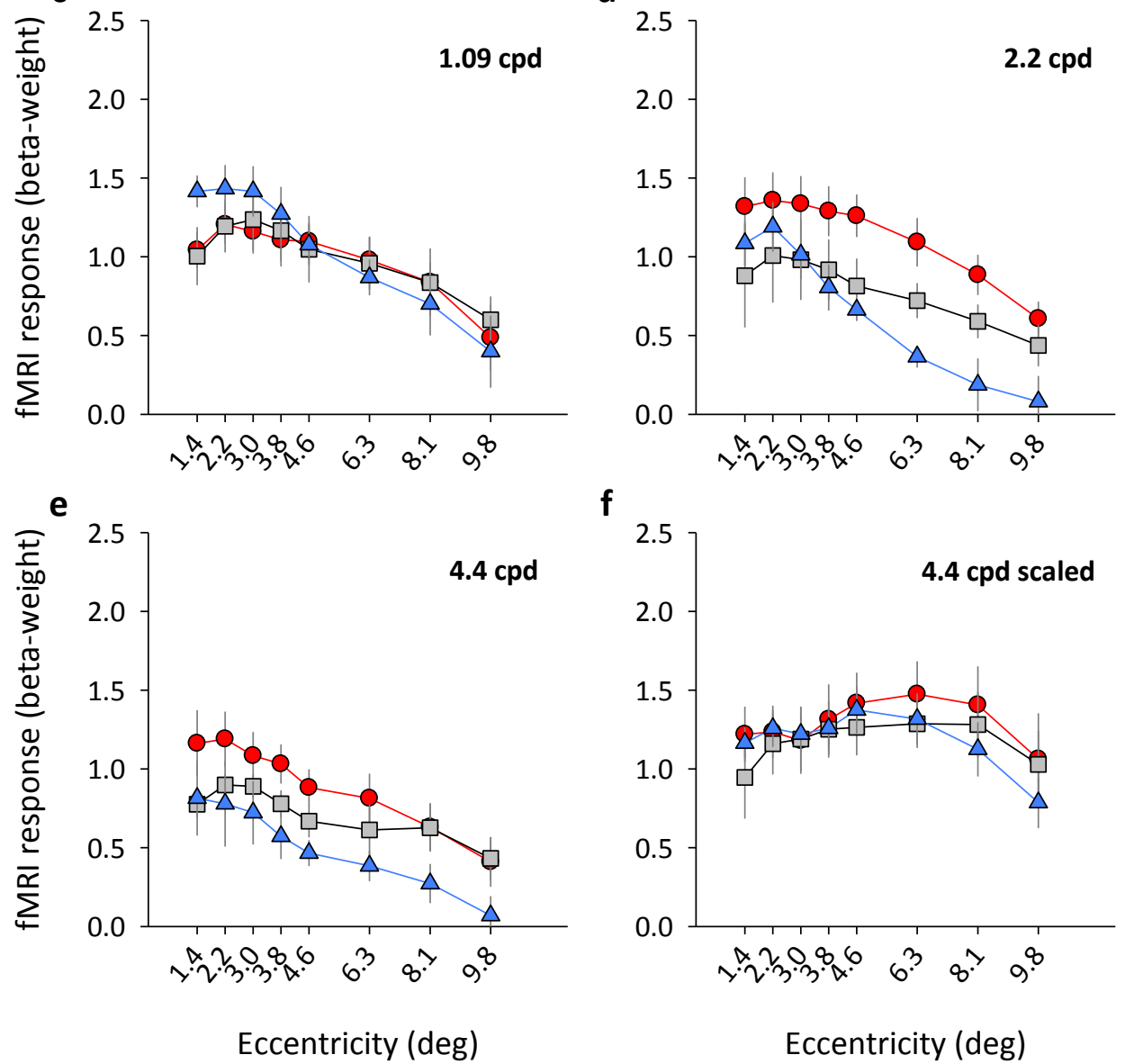

f

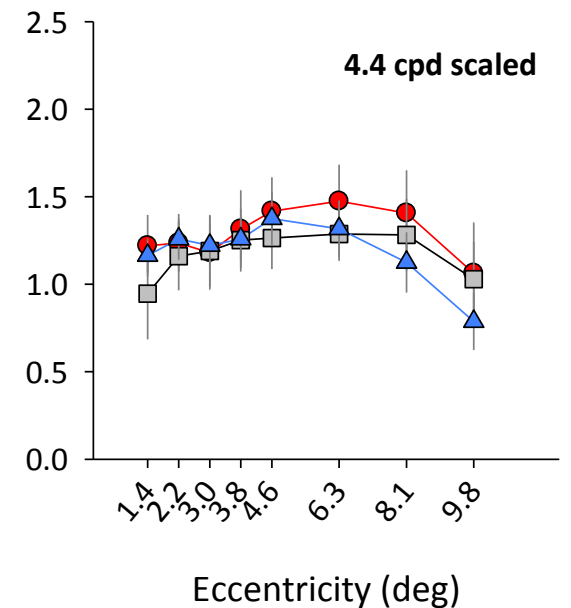

Figure 3.5: Sensitivity in VP as a function of visual field eccentricity

fMRI responses averaged over six hemispheres plotted as a function of visual field eccentricity for stimulation of the three post-receptoral pathways: L-M, L+M, and $S$, respectively. The subplots show eccentricitydependent responses at stimulus spatial frequencies of a) $0.27 \mathrm{cpd}, \mathrm{b}) 0.55 \mathrm{cpd}, \mathrm{c}) 1.09 \mathrm{cpd}, \mathrm{d}) 2.2 \mathrm{cpd}$, and e) $4.4 \mathrm{cpd}$, respectively. f) $4.4 \mathrm{cpd}$ scaled - here the stimulus comprised a central spatial frequency of $4.4 \mathrm{cpd}$, which is scaled along the visual field eccentricities according to the cortical magnification function, resulting in a spatial frequency of $0.16 \mathrm{cpd}$ at the periphery. The data are color coded according to the different stimulus conditions. Error bars indicate \pm s.e.m. The data shows that relative responses to the different stimuli across eccentricity depend on spatial frequency of the stimulus. 


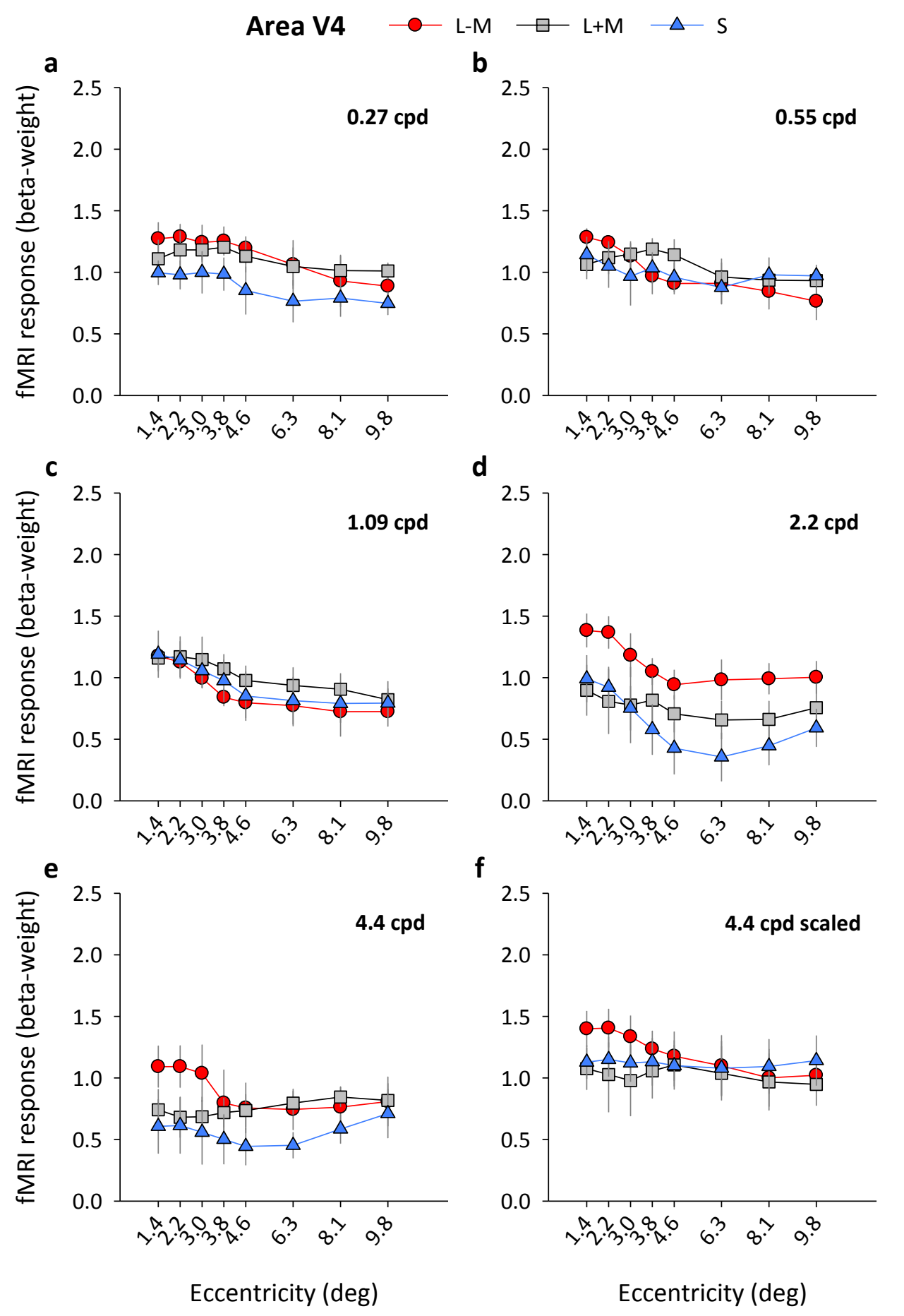

Figure 3.6: Sensitivity in V4 as a function of visual field eccentricity

fMRI responses averaged over six hemispheres plotted as a function of visual field eccentricity for stimulation of the three post-receptoral pathways: L-M, L+M, and $S$, respectively. The subplots show eccentricitydependent responses at stimulus spatial frequencies of a) $0.27 \mathrm{cpd}$, b) $0.55 \mathrm{cpd}, \mathrm{c}) 1.09 \mathrm{cpd}, \mathrm{d}) 2.2 \mathrm{cpd}$, and e) $4.4 \mathrm{cpd}$, respectively. f) $4.4 \mathrm{cpd}$ scaled - here the stimulus comprised a central spatial frequency of $4.4 \mathrm{cpd}$, which is scaled along the visual field eccentricities according to the cortical magnification function, resulting in a spatial frequency of $0.16 \mathrm{cpd}$ at the periphery. The data are color coded according to the different stimulus conditions. Error bars indicate \pm s.e.m. The data shows that relative responses to the different stimuli across eccentricity depend on spatial frequency of the stimulus. 
In summary we have measured chromatic and luminance responses in V1 and areas V2v, VP, and V4 in the ventral visual pathway as a function of visual field eccentricity and spatial frequency. It appears that red-green responses are well maintained in the near-peripheral visual field in V1, and to a certain extent in V2V and VP at low spatial frequencies. However, we observed a pronounced decline in red-green response with eccentricity even at low spatial frequencies in V4.

The spatial frequency tuning curves at different eccentricities in V1 are similar for both red-green and luminance mechanisms with $E_{2}$ values of 1.6 and 2.17 deg, respectively. However, for the blue-yellow mechanism, a relatively higher $E_{2}$ value of 4.6 deg was observed. For the blue-yellow mechanism, this corresponds to a slower increase in receptive field size with eccentricity in comparison to the other two mechanisms. In the discussion section, we will attempt to relate these findings to previous electrophysiological and psychophysical data, e.g. the low S-cone density in central retina. 


\subsection{Temporal frequency tuning of chromatic and luminance responses in human retinotopic visual areas}

\subsubsection{Temporal-frequency tuning curves in LGN}

Chromatic response properties of the human LGN were first studied only recently using fMRI (Mullen et al., 2008). According to this study, the LGN shows robust responses to stimulation of the red-green (or L-M cone opponent) pathway and, at equal cone-contrast, relatively weaker responses for stimulation of the luminance (or $L+M$ ), and blue-yellow (or S-cone opponent) pathways. I investigated how the responsivity of the LGN varies to stimulation of the chromatic (red-green and blue-yellow) and luminance pathways for a range of temporal frequencies $(2,4,6,8,10$, and $12 \mathrm{~Hz}$ ) using high cone-contrast (29\%) stimuli. In the experiments, we presented M-scaled contrast-reversing circular-grating stimuli in a pseudo-randomized block design.

Figure 3.7 presents the results of the region-of-interest (ROI) analyses, averaged across twelve hemispheres, for the chromatic and luminance stimulus conditions in LGN. Figure 3.7a shows location and size of the two ROIs in the brain of a representative subject; Figure 3.7b shows the temporal frequency tuning curves, i.e. the LGN's response amplitude as a function of temporal frequency. In the plot we can see that the red-green response is well maintained up to the maximum temporal frequency $(12 \mathrm{~Hz})$. For luminance and blue-yellow stimuli, the responses are weaker at $2 \mathrm{~Hz}$ and peak at $4 \mathrm{~Hz}$, slightly decreasing at higher temporal frequencies.

Because the frequency axis is logarithmically scaled, the graph resembles a Bode plot, and we have fitted two separate linear functions to the tuning data. The first line connected data at 2 and $4 \mathrm{~Hz}$ (low frequency or LF curve, shown as solid line), the second was fitted through the set of data between 4 and $12 \mathrm{~Hz}$ (high frequency or HF curve, shown as dotted line). The slopes of the two lines describe the putative piece-wise trends present in the tuning data. A positive slope corresponds to an increase in $\mathrm{fMRI}$ response as a function of temporal frequency, i.e. a high pass behavior, whereas a negative slope corresponds to a low pass. For the LGN, the slopes of the fitted HF curve were small (negative) and nonsignificant for all stimulus conditions, meaning that the BOLD responses neither increased 
nor declined with increasing temporal frequency. Table 3.2 summarizes the slope values as well as their significance values for the temporal-frequency tuning curves.

a

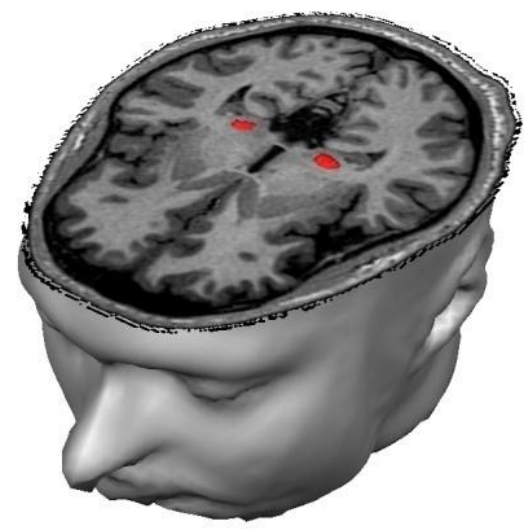

b

C

$\because \mathrm{L}-\mathrm{M} \square \mathrm{L}+\mathrm{M} \backsim \mathrm{S}$
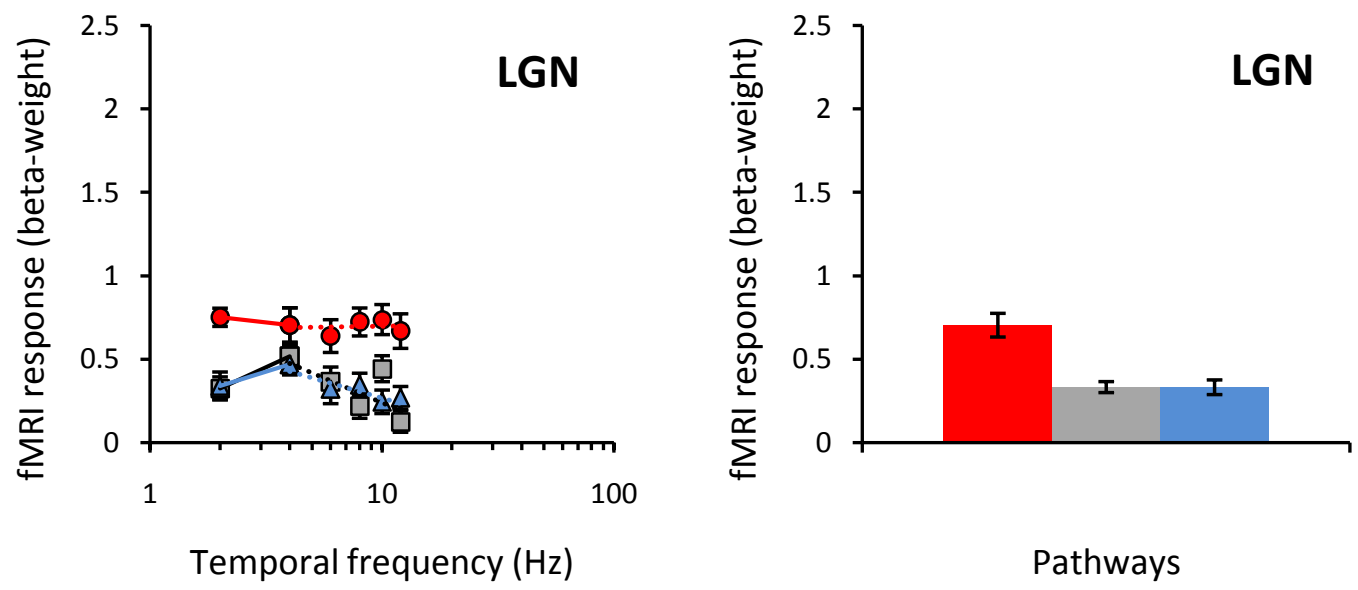

Pathways

Figure 3.7: Temporal frequency tuning curves in LGN

a) $3 D$ rendering of the clusters (red) representing the LGN for a representative subject. b) fMRI responses averaged over twelve hemispheres as a function of temporal frequency for stimulation of the three postreceptoral pathways: L-M, L+M, and S, respectively. Solid lines connect the 2 and $4 \mathrm{~Hz}$ points; the dotted lines are linear fits through data from 4 to $12 \mathrm{~Hz}$. Error bars indicate \pm s.e.m. c) Mean fMRI responses (across twelve hemispheres and six temporal frequencies) for the three stimulus types. Error bars indicate \pm s.e.m. 


\begin{tabular}{lccc}
\hline \hline Stimulus & $\mathrm{S}_{\mathrm{LF}}$ & $\mathrm{S}_{\mathrm{HF}}$ & $\mathrm{p}_{\mathrm{HF}}$ \\
\hline $\mathrm{L}-\mathrm{M}$ & -0.023 & 0.001 & 0.86 \\
\hline $\mathrm{L}+\mathrm{M}$ & 0.095 & -0.035 & 0.19 \\
$\mathrm{~S}$ & 0.065 & -0.024 & 0.06 \\
\hline
\end{tabular}

Table 3.2: Slope values for the temporal frequency tuning curves in LGN

Slopes of the fitted curves. $\mathrm{S}_{\mathrm{LF}}$, and $\mathrm{S}_{\mathrm{HF}}$ correspond to the slopes of the fitted curves between data 2 and $4 \mathrm{~Hz}$, and set of points between 4 and $12 \mathrm{~Hz}$, respectively (see Figure 3.7). $p_{\mathrm{HF}}$ indicates the significance of $\mathrm{S}_{\mathrm{HF}}$. Negative slope values indicate low pass behavior.

LGN responsivity is highest to the red-green stimulus

Figure 3.7c shows the mean responses averaged across twelve hemispheres and six temporal frequencies, for the three stimulus conditions. Averaging across temporal frequencies allows for amplitude comparison across channels to gauge the general chromatic responsivity of LGN. Two-way (three stimulus types: red-green, luminance, blueyellow; six temporal frequencies: $2,4,6,8,10,12 \mathrm{~Hz}$ ) repeated-measures ANOVA, performed to ascertain the general chromatic sensitivity, showed a main effect of stimulus type $\left(\mathrm{F}_{(2,22)}=17.3 ; \mathrm{p}<0.001\right)$. Bonferroni corrected, pairwise comparisons of the stimulus types showed that the red-green response was significantly stronger than the luminance $(p<0.001)$ and blue-yellow response $(p<0.01)$. These results are consistent with the recent findings on the chromatic selectivity of the LGN (Mullen et al., 2008). We consider these results in relation to the firing rate of ganglion and LGN cells in the discussion. 


\subsubsection{Temporal frequency tuning curves in V1}

Figure 3.8 presents the results of the ROI analyses in V1, averaged across 12 hemispheres for chromatic and luminance conditions. Figure $3.8 \mathrm{a}$ shows the demarcation of V1 on a reconstructed cortical surface of the left hemisphere of a representative subject. The temporal frequency tuning curves are shown in Figure $3.8 \mathrm{~b}$. From the tuning curves it is evident that $\mathrm{fMRI}$ responses in V1 for the red-green stimulus are approximately constant up to $12 \mathrm{~Hz}$. This was confirmed by the small negative, non-significant slope value. V1 responded most strongly to the luminance stimulus at $2 \mathrm{~Hz}$; the luminance response then decreases at $4 \mathrm{~Hz}$ and remains approximately constant up to $12 \mathrm{~Hz}$. There is a steep decrease with temporal frequency for the blue-yellow pathway. Liu \& Wandell (2005) have previously reported that such differential responses between low and high temporal frequencies occur with low cone-contrast, blue-yellow stimuli, whereas at high conecontrast (>20\%), both low and high temporal frequencies elicit equally strong fMRI responses in V1. Here we show that despite the high cone contrast of the stimuli, there is still a prominent difference of the blue-yellow response between low and high temporal frequencies in V1. Table 3.3 summarizes the slope values and their significance values for the temporal frequency tuning curves in V1. 
a

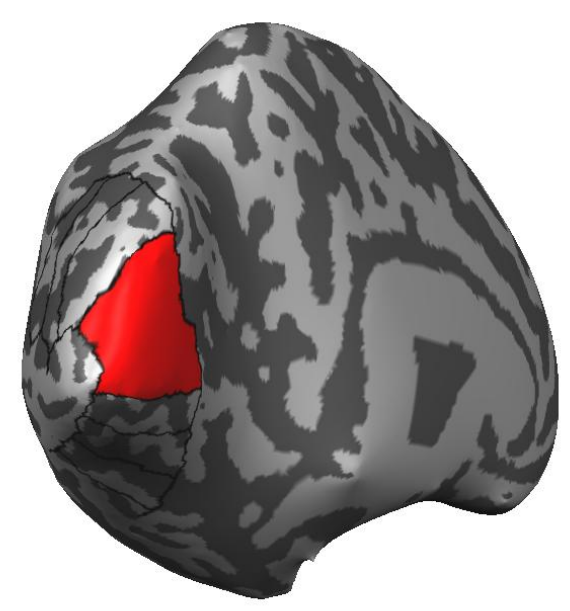

b

C

$\square \mathrm{L}-\mathrm{M} \sim \mathrm{L}+\mathrm{M} \sim \mathrm{S}$
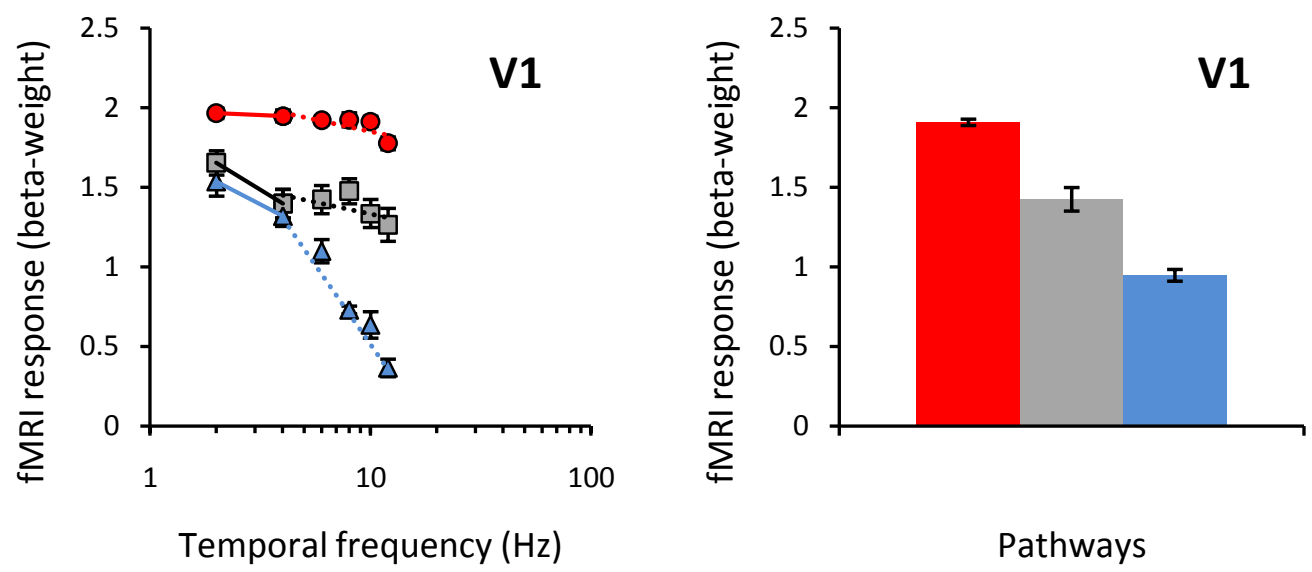

Figure 3.8: Temporal frequency tuning curves in V1

a) Retinotopic map of area V1 (red) on a reconstructed cortical surface of a representative subject. b) fMRI responses averaged over twelve hemispheres are plotted as a function of temporal frequency for stimulation of the three post-receptoral pathways: $L-M, L+M$, and $S$, respectively. The solid lines indicate first-order polynomial curve fit between temporal frequencies 2 and $4 \mathrm{~Hz}$, whereas the dotted lines indicate first-order polynomial curve fit through temporal frequency data between 4 and $12 \mathrm{~Hz}$. Error bars indicate \pm s.e.m. c) Mean fMRI responses (across twelve hemispheres and six temporal frequencies) are shown for three stimulus types. Error bars indicate \pm s.e.m. 


\begin{tabular}{lccc}
\hline Stimulus & $\mathrm{S}_{\mathrm{LF}}$ & $\mathrm{S}_{\mathrm{HF}}$ & $\mathrm{p}_{\mathrm{HF}}$ \\
\hline $\mathrm{L}-\mathrm{M}$ & -0.008 & -0.018 & 0.09 \\
\hline $\mathrm{L}+\mathrm{M}$ & -0.128 & -0.018 & 0.20 \\
$\mathrm{~S}$ & -0.108 & -0.119 & $0.001^{*}$ \\
\hline
\end{tabular}

Table 3.3: Slope values for the temporal frequency tuning curves V1

Slopes of the fitted curves are summarized in the table. $\mathrm{S}_{\mathrm{LF}}$, and $\mathrm{S}_{\mathrm{HF}}$ correspond to slopes of the fitted curves between data at 2 and $4 \mathrm{~Hz}$, and the set of points between 4 and $12 \mathrm{~Hz}$ (see Figure 3.8). $\mathrm{p}_{\mathrm{HF}}$ indicates the significance of the slope $\mathrm{S}_{\mathrm{HF}}$. Negative slopes correspond to low-pass characteristic. ${ }^{*} p \leq 0.05$.

\section{V1 responsivity is highest to red-green stimulus}

Figure $3.8 \mathrm{c}$ shows the mean responses averaged across twelve hemispheres and six temporal frequencies for the three stimulus conditions. Two-way (three stimulus types: redgreen, luminance, blue-yellow; six temporal frequencies: $2,4,6,8,10,12 \mathrm{~Hz}$ ) repeated measures ANOVA, performed to ascertain the general chromatic sensitivity characteristics, showed a main effect of stimulus type $\left(F_{(2,22)}=115.24 ; p<0.001\right)$. Bonferroni corrected pairwise comparisons of the stimulus types showed that red-green responses were significantly stronger than luminance $(p<0.001)$ and blue-yellow responses $(p<0.001)$. Also, the luminance response was significantly $(p<0.001)$ higher than the blue-yellow response. These findings are in accordance with previous studies (Kleinschmidt et al., 1996; Engel et al., 1997a; Liu \& Wandell, 2005; Wade et al., 2008), suggesting high responsivity of V1 to modulation of the red-green pathway.

Our findings suggest that high temporal frequency blue-yellow chromatic information is subject to filtering in the primary visual cortex (V1). However, high-temporal frequency redgreen information appears to be well maintained in this area, implying that major filtering occurs across the extrastriate areas. 


\subsubsection{Temporal frequency tuning curves in ventral visual areas: V2v, VP, and} V4

Figure 3.9a shows the location of the ventral visual areas on the reconstructed cortical surface of the left hemisphere of a representative subject. Temporal frequency tuning curves are shown in Figure $3.9 \mathrm{~b}-\mathrm{d}$, for the ventral areas V2v, VP and V4, respectively. The patterns of temporal frequency responsivity in the ventral areas to modulation of the redgreen, luminance and blue-yellow pathway are largely similar to that of V1 for temporal frequencies beyond $4 \mathrm{~Hz}$, i.e. for temporal frequencies beyond $4 \mathrm{~Hz}$, blue-yellow responses declined steeply with temporal frequency, whereas red-green and luminance modulations resulted in robust responses up to $12 \mathrm{~Hz}$. Table 3.4 summarizes the slope values as well as their significance values for the temporal frequency tuning curves in ventral visual areas. It is widely held that V4 is a candidate area for color processing and plays a critical role in human color perception (Zeki, 1983b; Kleinschmidt et al., 1996; McKeefry \& Zeki, 1997; Zeki \& Marini, 1998; Beauchamp et al., 1999; Wade et al., 2002; Brewer et al., 2005; Wade et al., 2008). Also, a few studies have shown that V4 is specialized to process slow color modulations (<4 Hz) (Liu \& Wandell, 2005). In accordance with these previous studies, the date indicates an amplification of color (red-green and blue-yellow) relative to luminance responses in VP and V4 at temporal frequency of $2 \mathrm{~Hz}$, as compared to higher frequencies. 
a

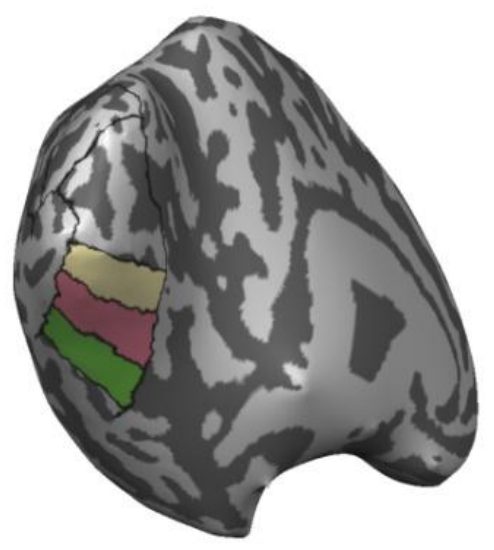

b

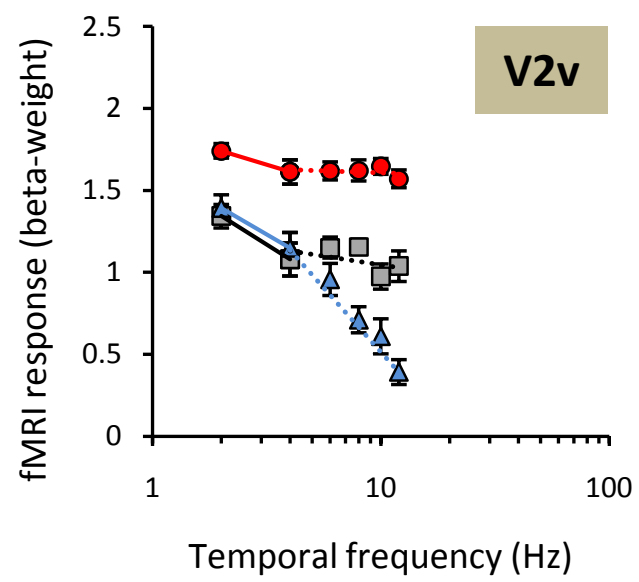

d
C

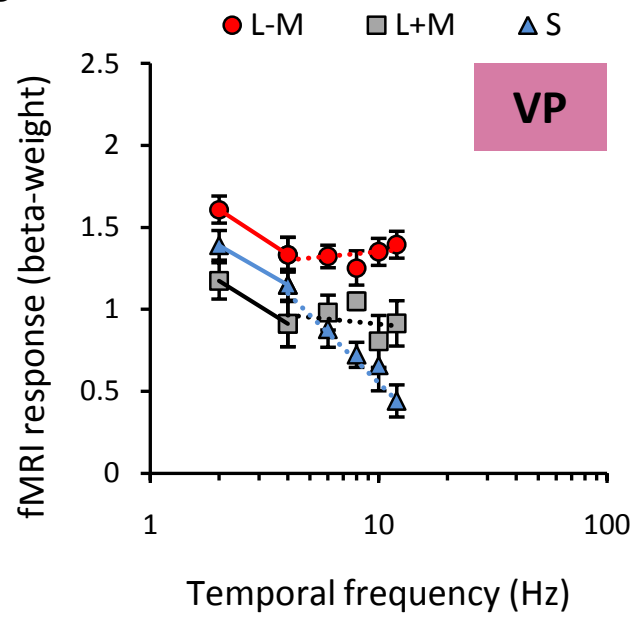

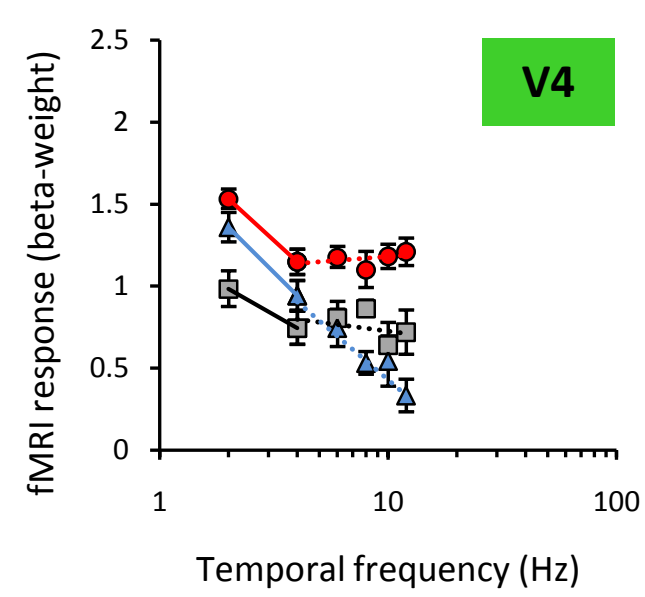

Figure 3.9: Temporal frequency tuning curves in ventral visual areas

a) Retinotopic maps of areas V2v, VP, and V4 are color coded on a reconstructed cortical surface of a representative subject. $\mathrm{fMRI}$ responses in V2v (b), VP (c), and V4 (d), averaged over twelve hemispheres, are plotted as a function of temporal frequency for stimulation of the three post-receptoral pathways: L-M, L+M, and $S$, respectively. The solid lines indicate first-order polynomial curve fit between temporal frequencies 2 and $4 \mathrm{~Hz}$, whereas the dotted lines indicate first-order polynomial curve fit through temporal frequency data between 4 and $12 \mathrm{~Hz}$. Error bars indicate \pm s.e.m. 


\begin{tabular}{clcccc}
\hline & Stimulus & $\mathrm{S}_{\mathrm{LF}}$ & $\mathrm{S}_{\mathrm{HF}}$ & $\mathrm{p}_{\mathrm{HF}}$ \\
\hline \multirow{2}{*}{$\mathrm{V} 2 \mathrm{v}$} & $\mathrm{L}-\mathrm{M}$ & -0.065 & -0.003 & 0.59 \\
& $\mathrm{~L}+\mathrm{M}$ & -0.132 & -0.013 & 0.35 \\
& $\mathrm{~S}$ & -0.122 & -0.093 & $0.001^{*}$ \\
\hline \multirow{2}{*}{$\mathrm{VP}$} & $\mathrm{L}$ & $\mathrm{L}+\mathrm{M}$ & -0.137 & 0.007 & 0.44 \\
\hline & $\mathrm{S}$ & -0.132 & -0.008 & 0.63 \\
\hline \multirow{2}{*}{ V4 } & L-M & -0.121 & -0.082 & $0.003^{*}$ \\
\hline & $\mathrm{L}+\mathrm{M}$ & -0.193 & 0.006 & 0.40 \\
\hline & $\mathrm{S}$ & -0.119 & -0.011 & 0.49 \\
\hline
\end{tabular}

Table 3.4: Slope values for the temporal frequency tuning curves in ventral visual areas Slopes of the fitted curves. $\mathrm{S}_{\mathrm{LF}}$, and $\mathrm{S}_{\mathrm{HF}}$ correspond to the line connecting 2 and $4 \mathrm{~Hz}$, and set of points between 4 and $12 \mathrm{~Hz}$, respectively (see Figure 3.9). $\mathrm{p}_{\mathrm{HF}}$ indicates the significance of the slope $\mathrm{S}_{\mathrm{HF}}$. Negative slope value indicates low-pass behavior. ${ }^{*} p \leq 0.05$.

\section{Overall responsivity of ventral visual areas}

Two-way (three stimulus types: red-green, luminance, blue-yellow; six temporal frequencies: $2,4,6,8,10,12 \mathrm{~Hz}$ ) repeated-measures ANOVA, computed for individual visual areas, showed a main effect of stimulus type in all ventral visual areas (V2v: $F_{(2,22)}=103.61$; $\left.p<0.001, V P: F_{(2,22)}=50.33 ; p<0.001, V 4: F_{(2,22)}=51.24 ; p<0.001\right)$. Bonferroni corrected pairwise comparisons of the stimulus types for each area showed that the red-green response was significantly stronger than the luminance and blue-yellow responses in V2v $(p<0.001), V P$ $(p<0.001)$, and $\mathrm{V} 4(p<0.001)$; only in area $\mathrm{V} 2 \mathrm{v}$ the luminance response was stronger than the blue-yellow response $(p<0.01)$.

In summary, there was a significant decrease in red-green, luminance as well as blueyellow responses from 2 to $4 \mathrm{~Hz}$. Furthermore, this decrease becomes more pronounced moving along the ventral pathway. However, red-green and luminance responses are maintained at temporal frequencies above $4 \mathrm{~Hz}$, contrary to our expectation. It is uncertain whether this a contrast-related effect as described by Liu \& Wandell (2005) in V1. 


\subsubsection{Temporal frequency tuning curves in dorsal visual areas: V2d, V3d, V3a, and MT}

Figure 3.10a shows the location of the dorsal visual areas on the reconstructed cortical surface of the left hemisphere of a representative subject. Temporal frequency tuning curves are shown in Figure $3.10 \mathrm{~b}-\mathrm{d}$, for areas V2d, V3d, V3a, and MT, respectively. The pattern of temporal frequency responsivity in $\mathrm{V} 2 \mathrm{~d}$ to modulations of the red-green, luminance and blue-yellow pathways is similar to its ventral counterpart, i.e. area V2v. Both areas $\mathrm{V} 3 \mathrm{~d}$ and $\mathrm{V} 3 \mathrm{a}$ show strong red-green and luminance responses at high temporal frequencies. A decline in blue-yellow responses with temporal frequency was observed in V2d, V3d, and in area V3a as well. The motion- sensitive area MT shows responses which increase with increasing temporal modulations of the red-green, luminance as well as blueyellow pathways. Table 3.5 summarizes the slope values as well as their significance values for the temporal frequency tuning curves in dorsal visual areas. 
a

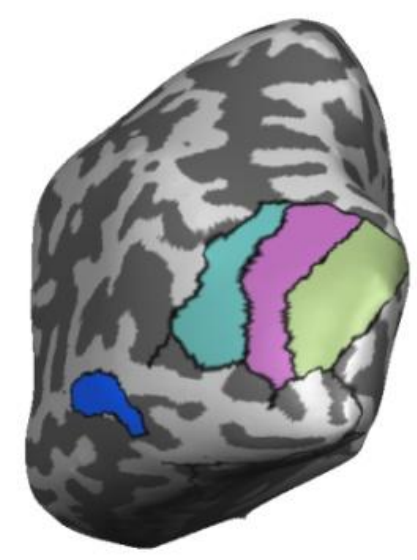

b

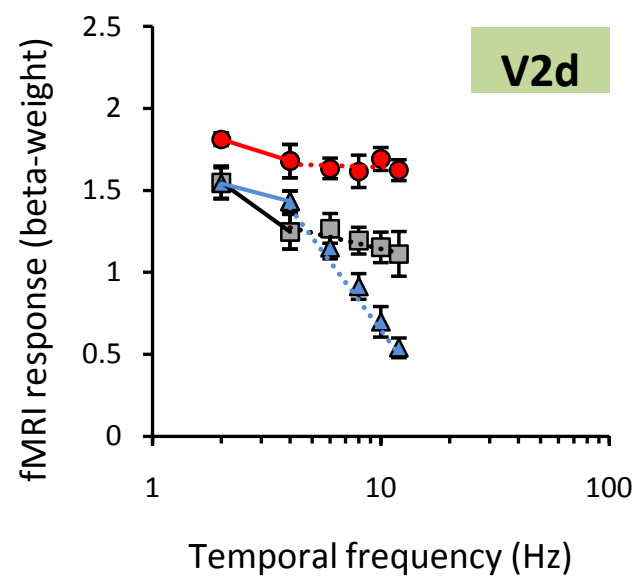

d

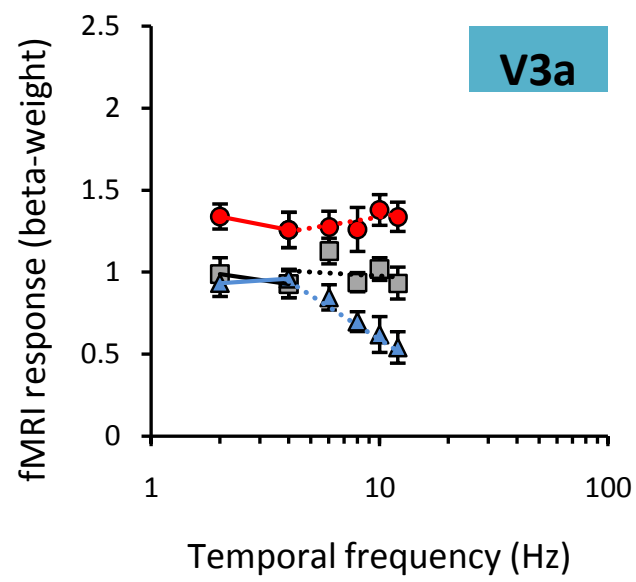

C

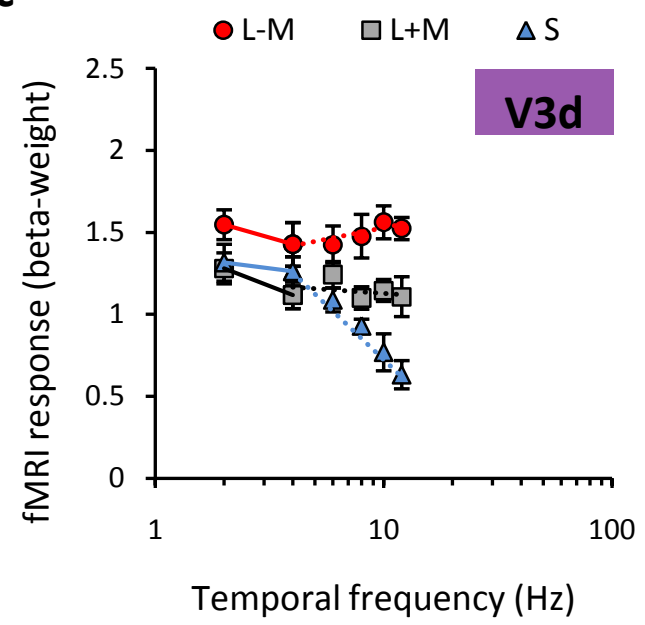

e

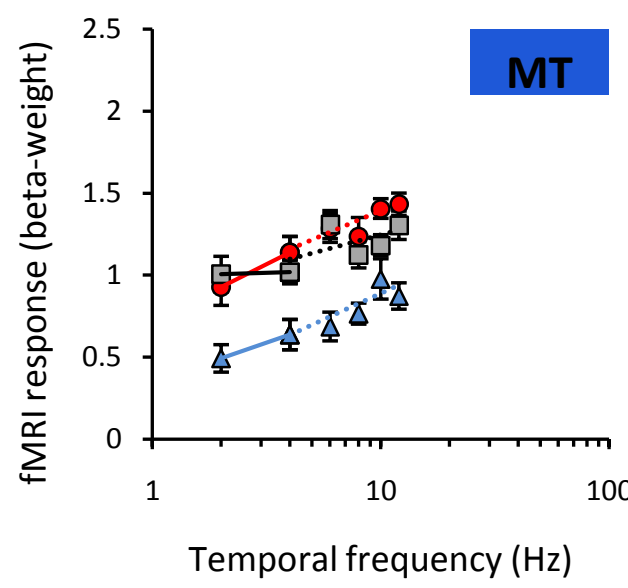

Figure 3.10: Temporal frequency tuning curves in dorsal visual areas

a) Retinotopic maps of areas V2d, V3d, V3a, and MT are color coded on a reconstructed cortical surface of a representative subject. fMRI responses in V2d (b), V3d (c), V3a (d), and MT (e), averaged over twelve hemispheres, are plotted as a function of temporal frequency for stimulation of the three post-receptoral pathways: L-M, L+M, and S, respectively. The solid lines indicate first-order polynomial curve fit between temporal frequencies 2 and $4 \mathrm{~Hz}$, whereas the dotted lines indicate first-order polynomial curve fit through temporal frequency data between 4 and $12 \mathrm{~Hz}$. Error bars indicate \pm s.e.m. 


\begin{tabular}{clcccc}
\hline & Stimulus & $\mathrm{S}_{\mathrm{LF}}$ & $\mathrm{S}_{\mathrm{HF}}$ & $\mathrm{p}_{\mathrm{HF}}$ \\
\hline \multirow{2}{*}{ V2d } & L-M & -0.067 & -0.003 & 0.69 \\
& L+M & -0.151 & -0.019 & $0.01^{*}$ \\
& $\mathrm{~S}$ & -0.055 & -0.112 & $0.001^{*}$ \\
\hline \multirow{2}{*}{ V3d } & L-M & -0.060 & 0.016 & 0.05 \\
& L+M & -0.082 & -0.006 & 0.61 \\
\hline \multirow{2}{*}{ V3a } & S & -0.026 & -0.079 & $0.001^{*}$ \\
\hline & L+M & -0.041 & 0.013 & 0.12 \\
\hline \multirow{2}{*}{ MT } & S & -0.031 & -0.005 & 0.78 \\
\hline & L-M & 0.013 & -0.053 & $0.001^{*}$ \\
\hline & S & 0.106 & 0.035 & $0.03^{*}$ \\
\hline
\end{tabular}

Table 3.5: Slope values for the temporal frequency tuning curves in dorsal visual areas

Slopes of the fitted curves are summarized in the table. $\mathrm{S}_{\mathrm{LF}}$, and $\mathrm{S}_{\mathrm{HF}}$ correspond to slopes of the fitted curves between data 2 and $4 \mathrm{~Hz}$, and set of data between 4 and $12 \mathrm{~Hz}$ (see Figure 3.10). $p_{\mathrm{HF}}$ indicates the significance of the slope $\mathrm{S}_{\mathrm{HF}}$. Negative slope value indicates that responses decline with increasing temporal frequency. ${ }^{*} p \leq$ 0.05

\section{Overall responsivity of dorsal visual areas}

Two-way (three stimulus types: red-green, luminance, blue-yellow; six temporal frequencies: $2,4,6,8,10,12 \mathrm{~Hz}$ ) repeated measures ANOVA, computed for individual visual areas, showed a main effect of stimulus type in all dorsal visual areas $\left(V 2 d: F_{(2,22)}=40.75\right.$; $\left.p<0.001, V 3 d: F_{(2,22)}=19.71 ; p<0.001, V 3 a: F_{(2,22)}=25.38 ; p<0.001, M T: F_{(2,22)}=44.15 ; p<0.001\right)$. Bonferroni corrected pairwise comparisons of the stimulus types for each area showed that the red-green response was significantly stronger than the luminance and blue-yellow responses in $\mathrm{V} 2 \mathrm{~d}(\mathrm{p}<0.01), \mathrm{V} 3 \mathrm{~d}(\mathrm{p}<0.01)$, and $\mathrm{V} 3 \mathrm{a}(\mathrm{p}<0.01)$. In $\mathrm{MT}$, the luminance responses were equally strong compared to red-green responses. This amplification of luminance response can be accounted for by the high selectivity of MT neurons to luminance modulation. 


\subsubsection{Summary of temporal frequency tuning curves}

Histograms summarizing the temporal tuning properties of the primary, ventral, and dorsal cortical visual areas are shown in Figure 3.11. In the first panel (Figure 3.11a) are the distribution of slopes $\mathrm{S}_{\mathrm{LF}}$, i.e. the slope of the fitted line between 2 and $4 \mathrm{~Hz}$. The values indicate that for color modulations (red-green and blue-yellow), the optimum temporal frequency is $2 \mathrm{~Hz}$ for all the ventral visual areas. The left side of the panel (left of the gray shading) suggests that dorsal areas do not show such preferential color responses at low temporal modulations. The second panel (Figure 3.11b) shows the distribution of slopes $\mathrm{S}_{\mathrm{HF}}$, (i.e. slope of the fitted lines through data between 4 and $12 \mathrm{~Hz}$ ). Given the high conecontrast values, both red-green and luminance stimuli elicit equally strong responses for temporal modulation frequencies up to $12 \mathrm{~Hz}$ in all visual areas (except for V2d). In MT, however, the red-green response increased significantly with temporal frequency. Blueyellow responses decline significantly with increasing temporal frequency in all areas except MT, whereas in MT, we see an increase in blue-yellow response, similar to that of red-green. 
a $\square \mathrm{L}-\mathrm{M} \quad \mathrm{L}+\mathrm{M} \backsim \mathrm{S}$

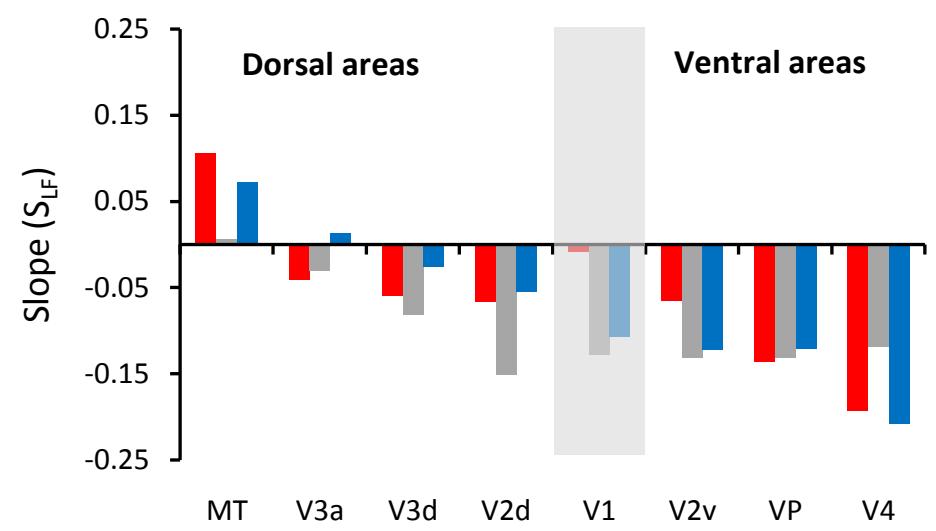

Visual areas

b $\square \mathrm{L}-\mathrm{M} \square \mathrm{L}+\mathrm{M} \square \mathrm{S}$

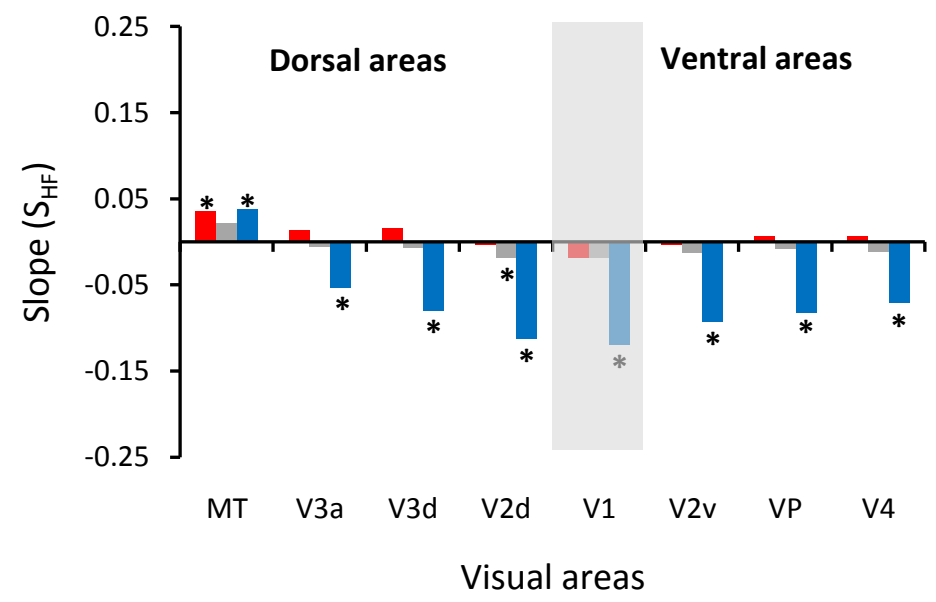

Figure 3.11: Distribution of slopes of the temporal frequency tuning curves across dorsal and ventral visual areas

a) Slopes of the fitted lines between temporal frequencies 2 and $4 \mathrm{~Hz}$, for the three post-receptoral pathways: L-M, L+M, and S, respectively. b) Slopes of the fitted lines through data between 4 and $12 \mathrm{~Hz}$. ${ }^{*} p \leq 0.05$.

\subsubsection{Cluster analysis of visual areas}

The result of the cluster analysis based on the slopes of the temporal frequency tuning curves (for all three stimulus conditions) is shown in Figure 3.12 in terms of a dendrogram. Visual areas showing similar temporal frequency tuning characteristics belong to one cluster. Areas V1, V2v, and V2d were clustered together as they all showed approximately 
an all-pass temporal frequency tuning characteristic to red-green and luminance modulations, and low-pass characteristics for blue-yellow modulations. Because these areas are located in the medial surface of the occipital cortex, the cluster comprising them is referred to as MO (medio occipital). Areas VP and V4 are clustered together into a VO (ventral occipital) cluster because of their high preferential sensitivity to color as compared to luminance modulations at $2 \mathrm{~Hz}$. Areas V3d and V3a are grouped together in cluster DO (dorsal occipital) because they respond well to high temporal frequency modulations. Area MT is singled out from all other areas in the dendrogram owing to its high sensitivity to increasing temporal frequency of the stimuli which reflects its specialized role in processing motion information.

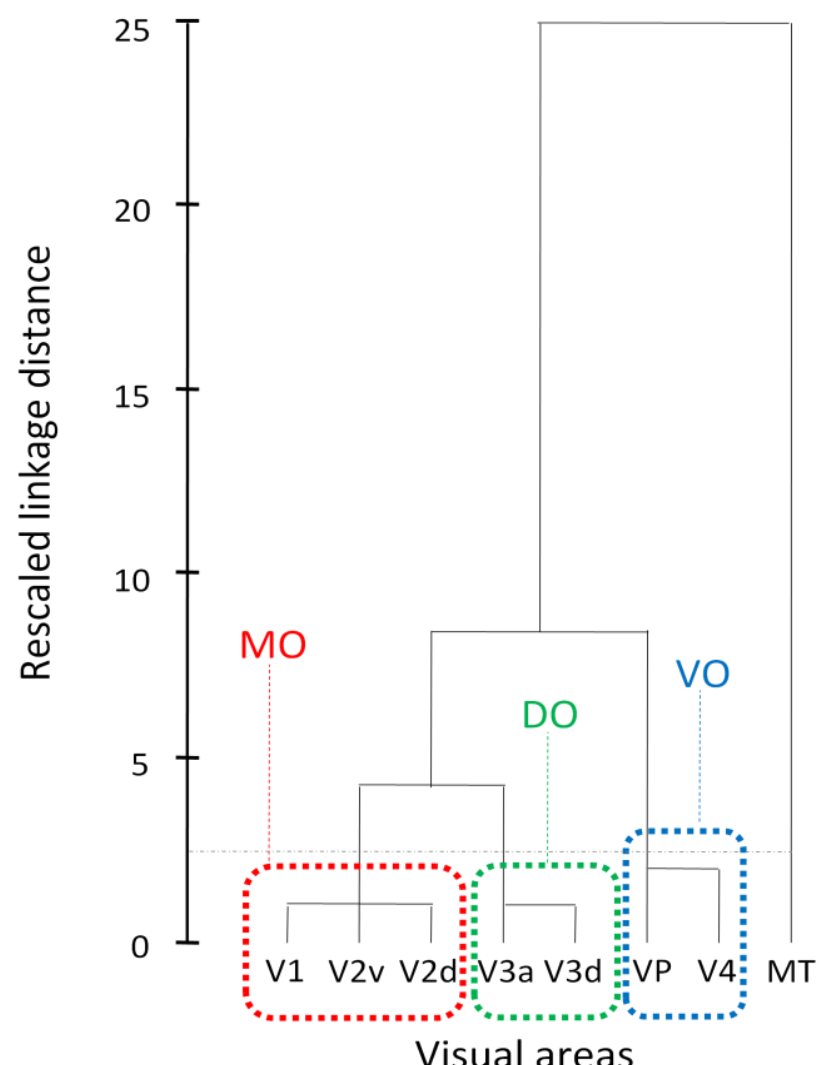

Figure 3.12: Clustering of retinotopic cortical visual areas

The dendrogram presents the output of the hierarchical cluster analysis as applied to categorize the eight cortical visual areas into groups based on similarities among their responses to color and luminance modulations at various temporal frequencies. The dendrogram above depicts the clustering of pattern, and degree of similarity (i.e. distances), among clusters. The squared Euclidian distance was used as a measure of response-profile similarity. The "between-group linkage" amalgamation schedule was used. Cluster analysis determined three groups: medial occipital, or MO (V1, V2v, and V2d), dorsal occipital, or DO (V3d, and V3a), and ventral occipital, or VO (VP, and V4). 
4

Discussion 


\subsection{Methodological aspects}

The experiments described in this thesis measured BOLD fMRI responses to stimuli designed to selectively stimulate the different afferent (retino-geniculo-cortical) pathways: red-green, luminance, and blue-yellow. In common with a previous approach (Kleinschmidt et al., 1996), fMRI responses were measured using a neurophysiological 'bottom up' rather than a 'top-down' approach, in which fMRI responses depend on behavioral or cognitive context.

It is critical for selective stimulation of the afferent pathways that calibration is such as to ensure adequate isolation of a given pathway. There are two critical aspects to calibration. One is the choice of 2 or 10 deg cone fundamentals (spectral sensitivities of the cones and $V_{\lambda}$ function) as a basis for stimulus calibration. These fundamentals differ mainly at short-wavelengths, and calculation of the red-green stimuli is little affected by the choice. However, on the other hand blue-yellow stimuli are affected; for example, an S-cone input to the MC-pathway was postulated (Chatterjee \& Callaway, 2002) based on parafoveal measurements using the 2 deg cone fundamentals, and this was later found to be artifactual (Sun et al., 2006). In the present experiments 10 deg cone fundamentals were used. However, this issue is not straightforward, since a large proportion of cortex is devoted to central vision ( $\pm 2-3 \mathrm{deg}$ ), and so the choice of a given fundamental set is a compromise. This is particularly the case for MT responses, because MT is highly sensitive to luminance contrast. There have been reports of MT fMRI responses attributed to S-cones (Wandell et al., 1999), who also used the 2 deg cone fundamentals. However, single cell measurements have not found evidence of S-cone input to MT (Gegenfurtner et al., 1994; Riecansky et al., 2005).

In the current experiments, responses to luminance modulation were generally weak, especially in comparison to red-green responses, so an artifactual red-green fMRI response due to luminance artifacts is unlikely. The only possible exception might be a blue-yellow response observed in $\mathrm{MT}$, wherein luminance responses became dominant and it cannot be excluded that the blue-yellow responses observed could have an artifactual origin.

A further critical issue in calibration is the inter-individual variability in subjects' luminosity function $\left(V_{\lambda}\right)$. Subjects' luminance matches (Anstis \& Cavanagh, 1983) for the red, green and blue lights were tested and found to be close to the standard observer. The main 
reason for inter-individual variability in $V_{\lambda}$ is inter-individual variability of the proportion of $\mathrm{M} / \mathrm{L}$ cones, which would mainly affect the selectivity in the red-green stimulus condition. Because, again, luminance responses were generally weak compared to red-green responses, contamination of measured red-green responses by a luminance artifact is unlikely.

In the present experiments, colors were modulated around different mean chromaticities depending on the pathway to be isolated. This permits using relatively high cone-contrasts. Other investigators have modulated about the white mean point (Vanni et al., 2006; Mullen et al., 2007). If one chooses to define the three pathway-selective stimuli in the DKL space (Derrington et al., 1984), as is the case with Mullen and Vanni (Mullen et al., 2007; Vanni et al., 2006), then the maximum achievable cone-contrast in the case of the L-M stimulus is often limited because of the less extent of the color gamut available for the display systems. In terms of pathway isolation, the mean chromaticity used is not thought to affect psychophysical results. This may not be the case if the object of the study is more cognitive aspects of color perception.

In an extensive series of preliminary experiments not reported here, I made measurements in which individual conditions (e.g. different temporal frequencies) were presented in different runs. Also, some measurements were made with stimuli modulated about equal energy white mean point. The general pattern of results obtained was similar to those described in the results sections. However, use of high cone-contrast stimuli and the pseudo-randomized stimulus block design, as described, yielded the most reliable results with the least inter-individual variability, and these experiments form the basis of the results section. 


\subsection{Dependence of chromatic and luminance responses on visual field eccentricity and spatial frequency}

Psychophysical sensitivity to chromatic modulation (especially red-green) declines more steeply toward the peripheral visual field (Anderson et al., 1991; Mullen, 1991; Stromeyer et al., 1992; Mullen \& Kingdom, 1996; Mullen \& Kingdom, 2002; Mullen et al., 2005) than does luminance sensitivity. Different models of midget ganglion cell (responsible for red-green color vision) receptive fields have been proposed to account for the deterioration of sensitivity to red-green color variations with eccentricity. However, recently, it has been shown through electrophysiological measurements that sensitivity loss does not have a retinal locus; it occurs post-retinally (Martin et al., 2001). Cortical mechanisms have been thought to be involved in the sensitivity loss. In the Spatial Frequency Experiment, eccentricity-dependent chromatic (L-M cone-opponent and S-cone pathways) and luminance $(L+M)$ pathways responses were measured in the primary visual cortex (V1) as well as in extrastriate visual areas. The interaction of eccentricity-dependent responses with spatial frequency was also studied. Through the eccentricity-dependent chromatic responses evident in several visual areas, it was possible to locate a putative neural locus underlying the steeply decline in psychophysical sensitivity to red-green chromatic modulation in the periphery.

\subsubsection{Psychophysical sensitivity loss to peripheral chromatic modulation does not have a neural correlate in V1}

Although distinct spatial distributions of $f M R I$ responses for stimulation of the L-M coneopponent (red-green), luminance $(L+M)$ and S-cone (blue-yellow) pathways were observed in V1, there was no loss in response as a function of eccentricity. This is evident in Figure $3.1 a$ and $b$, where the spatial frequencies of the grating pattern were 0.27 and $0.55 \mathrm{cpd}$, respectively. The reason to consider responses at these spatial frequencies was determined by psychophysical experiments which showed that peak cone-contrast sensitivity across eccentricity for chromatic and luminance mechanisms occur around these spatial frequencies (Mullen \& Kingdom, 2002; Mullen et al., 2005). Moreover, the choice of these 
spatial frequencies (which are too low to generate significant chromatic aberration) allows for comparison of results with two previous fMRI studies (Vanni et al., 2006; Mullen et al., 2007) concerned with eccentricity-related chromatic responses in V1. These studies showed significant responses to red-green modulation for the fovea and claimed a weak decline in response amplitude at higher eccentricities.

In contrast to these previous studies, we found little evidence of a decline in response with eccentricity (Figure 3.1a and b), and plausible reasons for this discrepancy are discussed in the introductory chapter (see section 1.6.2). It should also be stressed that the peripheral decline in sensitivity seen psychophysically is a log unit or more in magnitude. The relation between fMRI signal and psychophysical effect is uncertain, but decreases in fMRI response amplitude with eccentricity in these previous studies have been of much smaller amplitude. From our results it is clear that much of the retinal chromatic output is well represented in the primary visual cortex (V1) and V1 cannot be accounted as a neural substrate for the psychophysically observed chromatic sensitivity loss across eccentricity.

\subsubsection{Interaction of the stimulus spatial frequency with eccentricity- dependent response in $\mathrm{V} 1$}

Responses were measured at various spatial frequencies of the grating stimulus, and the results suggest a considerable degree of interaction between eccentricity-dependent responses and spatial frequency (Figure 3.1a-e). While lower spatial frequencies elicited stronger responses at higher eccentricities, as spatial frequency increased, there was a systematic shift in peak response toward lower eccentricities. This is indicative of the fact that with eccentricity there is an increase in average receptive field size and decrease in the density of the neural representation. However, distribution patterns for red-green, luminance, and blue-yellow responses were quite distinct. The differential distribution of red-green and blue-yellow responses in V1 for the M-scaled grating stimulus (Figure 3.1f) suggests that these pathways have distinct neurophysiological origins, and hence the spatial frequency sensitivity profiles might differ between them.

Anatomical and physiological studies (in non-human primates) have provided evidence that blue-yellow cone-opponency passes through a separate pathway originating in the 
small bistratified ganglion cell type of the retina. There is also evidence suggesting that this pathway remains distinct through the koniocellular layers of the LGN and to the blobs of V1 (Chatterjee \& Callaway, 2003). By contrast, the red-green cone-opponency originates in the midget $(P C)$ cells of the retina that predominate in the central visual field. However, little physiological data is available on the anatomical distribution of the specialized small bistratified ganglion cells across the visual field; presumably their density change in parallel to S-cone density.

One criterion to verify if the red-green and blue-yellow pathways have different neurophysiological origins would be to measure spatial frequency tuning properties across different eccentricities in V1 (in our human subjects) and this may complement previous psychophysical experiments (Vakrou et al., 2005). Different optimal spatial frequencies were obtained at different eccentricities for the red-green, luminance and blue-yellow mechanisms. In our experiments, high optimal spatial frequencies for the red-green and luminance stimuli were observed at the fovea, however with eccentricity there was a rapid decline in optimum spatial frequency (Figure 3.2a). This rapid variation in the scale (optimum spatial frequency) with eccentricity is evident from the small $E_{2}$ value (Figure $3.2 b)$ for the red-green response.

A slightly higher $E_{2}$ was estimated for the luminance mechanism. In contrast to red-green and luminance mechanisms, a large $E_{2}$ value is obtained for the blue-yellow mechanism. This disparate variation in spatial scale (optimum spatial frequency) for red-green and luminance versus blue-yellow pathways with eccentricity may indicate distinct neurophysiological origins.

It has been proposed (Mollon, 1982) that opponent systems which receive S-cone input may be the manifestation of an evolutionarily older color pathway that is shared by most (dichromatic) mammals, prior to the more recent acquisition of a second system based upon the differentiation of cones into long- (or L-cone) and middle-wavelength (M-cone) sensitive receptors. These different evolutionary origins are echoed in the different genetic coding of S-cone versus L- and M-cone photopigments (Nathans, 1999), physiological processing in separable anatomical pathways (Dacey \& Lee, 1994) and retinal distribution of cones. S-cones are absent in the tritanopic fovea, increase rapidly relative to $\mathrm{M}$ - and $\mathrm{L}$-cones 
up to 7 deg eccentricity, and then stabilize and remain relatively constant beyond this point (Curcio, 1991).

Some psychophysical observations suggest that chromatic (red-green and blue-yellow) sensitivity in the peripheral visual field can be made equal with foveal sensitivity by incorporating different spatial scaling factors for individual pathways (Vakrou et al., 2005; Hansen et al., 2009). However, it should be stressed that this suggestion from psychophysics is at variance with the original M-scaling concept, which was directly based on density of retinal elements and their representation in V1. How far the different $E_{2}$ value for the $S$ cone pathway is directly related to the low S-cone density in central retina remains uncertain.

Overall, although these findings provide evidence that, the differential distribution of red-green and blue-yellow cone-opponency across the visual field in human vision stem from pathways which have distinct neurophysiological origins, it appears that eccentricityrelated red-green sensitivity loss does not occur in V1.

As mentioned in the introduction, PC cell responses to luminance and chromatic gratings tend to have similar spatial frequency cutoff limits. This also appeared to be the case in our V1 measurements, where spatial frequency tuning curves for L-M and L+M appear similar. This is a further instance in which V1 responses appear to match retinal physiology rather than perceptual results.

\subsubsection{Does eccentricity-dependent psychophysical chromatic sensitivity loss have neural correlates in the extrastriate visual areas?}

Previous $\mathrm{fMRI}$ reports have shown that extrastriate area V4 and areas ventral to it elicit robust responses to chromatic over luminance stimuli (Kleinschmidt et al., 1996; Liu \& Wandell, 2005). Though regarded as controversial, there is also a suggestion that conscious perception of color is linked to these ventral visual areas (Murphey et al., 2008). Furthermore, the representation of visual field eccentricities in V4 is comparatively coarser than other visual areas. From this, it is likely that substantial discrepancies in processing chromatic information emerge among ventral visual areas as the information passes through these hierarchically organized areas. In this thesis, the analysis pertaining to the 
spatial distribution of chromatic and luminance response was therefore restricted to the ventral areas (V2v, VP, and V4). In addition, in the Spatial Frequency Experiments, by setting the temporal modulation frequency of the grating stimuli to low values (e.g. $2 \mathrm{~Hz}$ ), any possible confounding effects of high temporal frequencies on ventral visual areas were excluded.

Based on the present results, the response properties of $\mathrm{V} 2 \mathrm{v}$ bear a close resemblance to V1 data, meaning there was no loss in both chromatic (red-green and blue-yellow) and luminance responses as a function of eccentricity in V2v. As in V1, there was also a foveal preference for high spatial frequency and peripheral preference for low spatial frequencies in V2v. The spatial frequency tuning curves and slightly lower $E_{2}$ values, however, suggest that the average receptive-field size in $\mathrm{V} 2 \mathrm{v}$ increases as a function of eccentricity at a faster rate than in $V 1$. Different $E_{2}$ values were estimated for red-green, luminance and blueyellow mechanisms, respectively. While these three pathways are known to be segregated in V1, this could also hold true for area V2v. The findings support the view that visual information processing in V2v occurs at a progressively coarser scale, which is further consolidated in VP and V4.

In VP the data indicate a decline in response across eccentricity even at the lower spatial frequency of $0.55 \mathrm{cpd}$ (regarded as an optimal spatial frequency resulting in no psychophysical sensitivity loss across eccentricity (Mullen et al., 2007)). This is in contrast with the response profile observed in V1, wherein an increase in response across eccentricity can be observed for the same spatial frequency.

Finally, in area V4, for all spatial patterns studied here, there was a tendency of the fMRI response to decrease with eccentricity. The M-scaled stimulus, that elicited an approximately even distribution of responses across eccentricity in early areas (V1, V2v, and VP), also failed to produce an equal response across eccentricity in V4. This clearly indicates that visual information in V4 is processed on a different spatial scale in comparison to other areas. In the past it has been shown that receptive field size is smallest in human V1 and increases both as a function of eccentricity and from V1 to extrastriate areas with the largest receptive fields in V4 (Henriksson et al., 2008). The present findings are consistent with these observations. One may therefore pose the question whether area V4 can account for the loss in psychophysical chromatic sensitivity across eccentricity. The poor 
Discussion

representation of the peripheral chromatic information shown here indeed favors the view that V4 has neural substrates for this sensitivity loss, which is also in line with the fact that V4 has a salient role supporting conscious perception of color. It remains uncertain whether the deterioration of ability to process chromatic information in V4 is specific to this modality; it could be that ventral cortical areas are specialized for processing of information from central retina, independent of its chromatic content. 


\subsection{Temporal frequency tuning of chromatic and luminance responses in human retinotopic visual areas}

Psychophysical sensitivity to chromatic (red-green and blue-yellow) modulation declines rapidly at temporal frequencies beyond $4 \mathrm{~Hz}$ (Kelly, 1983); sensitivity loss is thought to occur post-retinally, i.e. in cortical visual areas. Up to now, little is known about how the temporal resolution for chromatic information is set through cortical visual areas. Moreover, current fMRI studies have not yet reached a consensus regarding temporal frequency processing in visual areas. The purpose of the Temporal Frequency Experiment was to investigate functional response properties of human visual areas to stimulation of the chromatic (L-M cone-opponent or red-green and S-cone or blue-yellow) and luminance ( $L+M)$ pathways at different temporal modulation frequencies. BOLD $\mathrm{FMRI}$ responses to chromatic and luminance modulations (at equal cone-contrast) were measured in lateral geniculate nucleus (LGN) and cortical visual areas (V1, V2v, V2d, VP, V3d, V3a, V4, and MT). The results show a distinct temporal frequency tuning of chromatic and luminance processing in visual areas.

\subsubsection{Does high temporal frequency chromatic information pass through LGN?}

The LGN is the primary thalamic nucleus linking the retina to the primary visual cortex (V1). So, it is natural to ask how LGN responds to chromatic and luminance modulations at different temporal frequencies. There is evidence, from single unit electrophysiology recordings in macaques that the LGN responds to high temporal frequency chromatic and luminance modulation (Hawken et al., 1996; Solomon et al., 1999), as do retinal ganglion cells (Lee et al., 1990); see also Figure 1.11. The experiments investigated the effect of temporal frequency up to $12 \mathrm{~Hz}$. The temporal frequency tuning curves (Figure 3.7b) suggest that red-green responses are well maintained up to $12 \mathrm{~Hz}$, whereas luminance and blueyellow responses peak at $4 \mathrm{~Hz}$, slightly decreasing thereafter. The results indicate that even though psychophysical sensitivity has declined steeply above $4 \mathrm{~Hz}$ (Kelly, 1983), LGN responses to red-green modulation persist to high temporal frequencies. Responses are less 
strong in amplitude to luminance and blue-yellow stimuli but also persist to high temporal frequencies. These findings are consistent with a recent fMRI study in LGN (Mullen et al., 2008), reporting robust red-green and blue-yellow responses to an $8 \mathrm{~Hz}$ stimulus.

While the main goal was to confirm temporal frequency tuning properties of the LGN, the overall responsivity of LGN to stimulation of the L-M cone-opponent (red-green), L+M (luminance), and S-cone (blue-yellow) pathways was also gauged. To do so, for each stimulus condition, the responses were averaged across six temporal frequencies used in the functional experiments (Figure 3.7c). In agreement with a previous report (Mullen et al., 2008), it is found that LGN activation is significantly greater for red-green than for the luminance and blue-yellow stimuli, supporting the same arguments provided by Mullen (Mullen et al., 2008) to interpret these findings. The significant difference between redgreen and blue-yellow response is likely to reflect, at least in part, the wide difference in the numbers of neurons activated by each stimulus. L-M cone-opponency is supported by the primate PC-cells of the LGN, which respond optimally to red-green stimulus (Derrington et al., 1984; Lee et al., 1990; Solomon \& Lennie, 2005) and form the large majority of the cells in the LGN (Shapley \& Perry, 1986). S-cone opponency, on the other hand, is supported in the primate LGN by a much smaller group of sparse, specialized neurons (Derrington et al., 1984; White et al., 1998), reflecting the low proportion of S-cones in the retinal cone population, $\sim 7 \%$ (Curcio et al., 1991). The lower discharge rate of koniocellular (KC)-cells compared with PC-cells (White et al., 2001), notably seen in the 'Blue-OFF' cells (Solomon \& Lennie, 2005), may also contribute to the relatively small LGN response to blue-yellow stimuli.

The relatively poor response to luminance may be accounted for by the poor responses of PC-cells to luminance modulation. This is made more specific in Figure 4.1. Figure 4.1a shows responses of a typical +M-L PC-cell to red-green modulation as a function of temporal frequency. The response ( $1^{\text {st }}$ harmonic) is vigorous when the modulation is present and almost zero in the absence of stimulation (maintained). The mean firing rate $\left(0^{\text {th }}\right.$ harmonic) is significantly increased by the stimulus (red and black lines). On the other hand, for luminance modulation (Figure $4.1 \mathrm{~b}$ ) there is a weak $1^{\text {st }}$ harmonic response (increasing rapidly at high frequency) but little change in mean rate compared to the resting level. There has been some discussion as to whether presynaptic mechanisms or spike generation 
account for the BOLD fMRI signal (Logothetis \& Wandell, 2004), but it would seem likely that little luminance fMRI signal derives from the PC-pathway. On the other hand, for MC-cell, luminance modulation significantly enhances mean rate (Figure 4.1c). This suggests that the luminance $\mathrm{fMRI}$ response may largely originate in the MC-layers of the LGN. Because cells in this pathway are much less numerous than in the PC-layers, this may account for the lower fMRI signal.

a

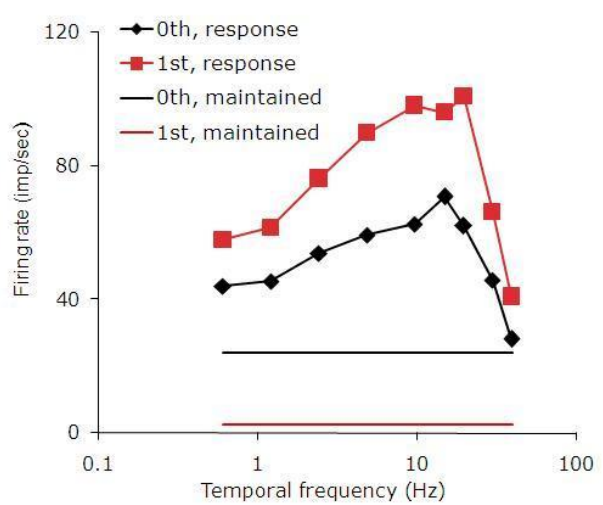

b

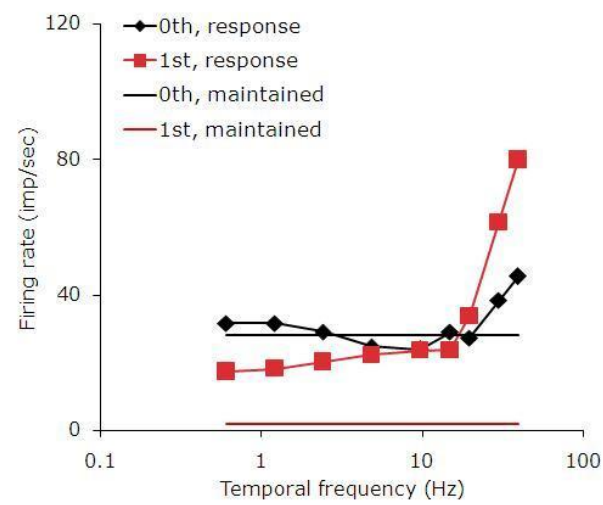

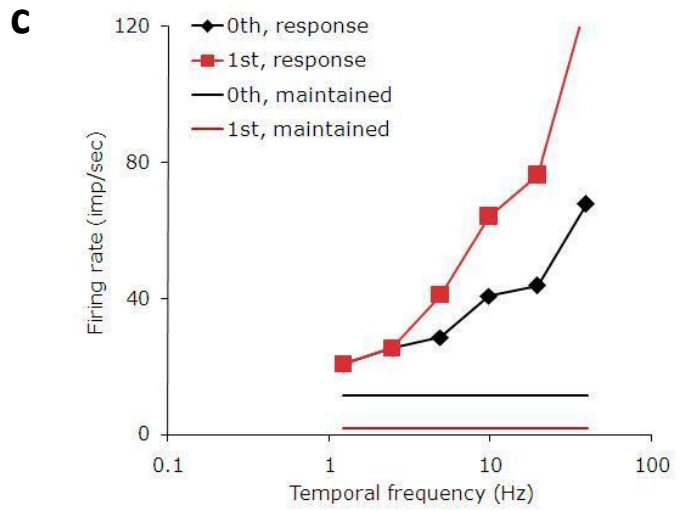

Figure 4.1: Effect on mean activity of luminance and chromatic modulation

a) Response of a +L-M PC-cell as a function of temporal frequency ( $50 \%$ contrast, 4 deg field) is shown.

$1^{\text {st }}$ harmonic amplitude and mean rate are shown, compared to values in control periods ( $0 \%$ contrast, shown as horizontal lines). Both response and mean rate are elevated by the chromatic modulation. Insofar as presynaptic activity and mean spike rate generate a BOLD response, a BOLD fMRI response may be generated. b) Similar conditions but for luminance modulation of same PC-cell. Luminance modulation responses are weaker, but there is little elevation of mean rate. Thus, if mean rate derives from synaptic activity arising from retinal input, little or no BOLD response may occur, since presynaptic activity (and spikes) are similar in both conditions. c) Responses (mean rate and modulated response) of MC-cell to luminance modulation. Mean rate is elevated. This may indicate that luminance BOLD responses in LGN arise from MC layers. Source: Lee B. B. (unpublished data). 
The present findings advance our understanding of chromatic information processing in human LGN. It was ascertained that red-green response dominates over luminance and blue-yellow response. In concordance with previous single unit data (Hawken et al., 1996), the results show that high temporal chromatic information is well preserved in human LGN, indicating that psychophysical sensitivity loss has no neural correlates in LGN. The differences in temporal tuning for chromatic stimuli are most likely to be a cortical phenomenon.

\subsubsection{V1 filters high temporal frequency blue-yellow chromatic information}

We observed robust red-green and luminance responses in V1 for temporal frequencies up to $12 \mathrm{~Hz}$ (Figure 3.8a). On the other hand, blue-yellow responses decreased significantly as a function of temporal frequency. For red-green (Jiang et al., 2007) and luminance responses, our results are in line with previous studies (Engel et al., 1997a; Liu \& Wandell, 2005; Mullen et al., 2008) showing strong response at high temporal frequencies. Furthermore, our data are also consistent with previous electrophysiological data showing that at least some V1 neurons respond strongly to red-green stimuli at high temporal frequencies (Gur \& Snodderly, 1997). Also, a single unit study (Hawken et al., 2001) has previously shown that for red-green modulation at the highest level of cone-contrast, V1 neurons show robust responses to an optimal temporal frequency of $12 \mathrm{~Hz}$ and then show very rapid attenuation of responses at about $20 \mathrm{~Hz}$.

Previous reports have suggested that raising temporal frequency (e.g. to $10 \mathrm{~Hz}$ ) selectively reduces the blue-yellow response in V1 over that of red-green (Engel et al., 1997a; Liu \& Wandell, 2005), but in the same studies it was shown that by increasing the cone-contrast of the stimulus it is possible to obtain equally strong responses even at high temporal frequencies. There was a significant decline in blue-yellow response with increasing temporal frequency in spite of the high cone contrast produced by the current experimental stimulus. One explanation for this discrepancy is that the studies from Liu and Wandell (2005) used the 2 deg rather than the 10 deg luminosity function $\left(V_{\lambda}\right)$ for calibration, which may have introduced a luminance artifact in the blue-yellow stimulus at high-contrast. Another issue which could be raised here is whether the decline in blue- 
yellow response with temporal frequency could potentially be associated to the interaction between spatial pattern of the grating stimulus and temporal frequency. Previously it has been shown that the $\mathrm{fMRI}$ response varies as a function of spatio-temporal frequency (Mirzajani et al., 2006). However, the grating stimuli used in our experiments were an approximate version of an M-scaled stimulus and can be regarded as an optimum spatial pattern. Hence, it is unlikely that the loss of blue-yellow responses at high frequency has occurred due to interaction between spatial and temporal factors.

One plausible explanation for the loss in blue-yellow response could be that V1 neurons which respond to blue-yellow stimuli may have poor temporal dynamics and an inefficient contrast gain-control mechanism as compared to their counterparts, i.e. neurons with responses to red-green and luminance stimuli, and robust responses to temporal frequencies at high cone-contrast (Hawken et al., 2001). Unfortunately, no single-unit data on temporal frequency tuning of cells in V1 with strong S-cone input is available.

The red-green and blue-yellow pathways are thought to have evolved at different times and for different purposes, and their substrates remain anatomically and genetically distinct (Mollon, 1991; Mollon 2002; Smithson \& Mollon, 2004). It has been suggested that the phylogenetically ancient blue-yellow pathway is temporally sluggish (Brindley, 1966), or has a long response latency (Smithson \& Mollon, 2004) as compared to the red-green pathway. These latter authors suggested that macaque V1 neurons receive blue-yellow signals with a delay in comparison to red-green due to a delay in arrival of blue-yellow signals in V1, related to the small diameter of the axons of the blue-yellow pathway between LGN and V1. However, it is unclear how this by itself could contribute to the high-frequency attenuation of the blue-yellow signals. In any event, this thesis provides evidence suggesting that V1 plays a major role in the psychophysical sensitivity loss to high temporal frequency blueyellow modulation.

\subsubsection{Temporal frequency tuning in extrastriate visual areas}

Consistent with V1 data, we observed that there was a significant decline in blue-yellow response with temporal frequency across the ventral visual areas (V2V, VP, and V4), further confirming the participation of ventral areas in psychophysical sensitivity loss to high 
temporal frequency blue-yellow modulation. The findings suggest that high temporal frequency chromatic (red-green and blue-yellow) information goes through increasing levels of filtering as it passes from V1 to V2V, VP, and V4, albeit this is apparent (especially for redgreen) only in terms of a differential response between 2 and $4 \mathrm{~Hz}$. This difference is pronounced in V4 suggesting its specialization for low-frequency chromatic information $1<4$ $\mathrm{Hz}$ ). Unlike blue-yellow, for red-green and luminance stimuli, no response change was observed between 4 and $12 \mathrm{~Hz}$. It is speculated that these responses might be due to a frequency doubling effect as reported previously by Lee et al. (1989). In comparison to the 2 $\mathrm{Hz}$ response, these responses were still significantly smaller in amplitude. The size of area V4 in our experiment was relatively large, so it is possible that some parts of area VO and V8 might have been included in our topographical definition of V4 (Brewer et al., 2005). It has been previously suggested that ventral visual areas (V4, VO) have a critical role in color perception and are highly selective for low temporal chromatic modulations (Liu \& Wandell, 2005). Our results are consistent with these findings. The current results provide substantial evidence that red-green high temporal information might be lost along the ventral pathway.

Unlike ventral areas, we saw an improvement in response to high temporal frequency stimuli (luminance and red-green) as information ascends from primary visual areas to dorsal extrastriate areas, i.e. V2d, V3d, to V3a. This enhancement in response is, however, well captured only between frequencies 2 and $4 \mathrm{~Hz}$ (blue-yellow information is filtered beyond $4 \mathrm{~Hz}$ ). This observation is in agreement with previous reports using chromatic and achromatic stimulation.

Area MT showed strongest luminance and red-green responses as compared to blueyellow and its temporal frequency tuning properties profoundly differ from all other areas. It has recently been suggested (Lee \& Sun, 2009) that the non-linear, frequency-doubled response of MC-cells is a means of enhancing motion signals for chromatic red-green targets close to isoluminance. This may correspond to the unsigned chromatic motion signal described by Dobkins and Albright (1995). The red-green fMRI signal we observed in MT may arise from this source. 


\subsubsection{Do 'Slow' and 'fast' information processing streams exist in the visual cortex?}

The present results indicate the existence of a hierarchy among visual areas which might belong to two previously described visual streams with functional specialization for slow and fast chromatic modulation, respectively. For example, Gegenfurtner and Hawken (1996) proposed two processing streams in the visual cortex for moving targets that differ mostly in their temporal characteristics. They suggested that the 'slow' channel has a high sensitivity for color, but does not code the velocity of the moving patterns veridically. On the other hand, the 'fast' channel has a high sensitivity to luminance-defined stimuli. Color variations are processed like small luminance variations without actually signifying color itself. Whereas area MT was thought very likely to be forming the neural substrate for the 'fast' channel, area V4 was hypothesized to be involved in the slow motion mechanism.

Results of this thesis are in agreement with the dual motion pathway view proposed by Gegenfurtner and Hawken (1996). It is interesting to note that it is implicit in this scheme that high temporal frequency luminance signals are also filtered out in the ventral pathway; the low critical flicker fusion (CFF) frequency for red-green is thus a consequence of lowpass filtering already occurring in the ventral stream. This again points to a distinction of the blue-yellow signal, in which high temporal frequencies are already lost in V1. If this is the case, it is unexpected that low temporal frequency filtering of the blue-yellow and red-green signals occurs at different cortical loci. 


$$
5
$$

Summary 
Visual performance in the detection of luminance patterns is generally well matched to the behavior of retinal ganglion cells across the retina, after stimuli are appropriately scaled with retinal eccentricity for the size of the cortical projection (M-scaling). However, this is not the case for chromatic vision. Chromatic sensitivity of a human observer is high in foveal vision, and - especially to red-green modulation - deteriorates towards the periphery of the visual field even after M-scaling. However, midget retinal ganglion cells, which are responsible for the detection of red-green change, respond equally well to chromatic modulation in the peripheral visual field as in the fovea. It has been postulated that central mechanisms are involved in the psychophysical sensitivity loss for color discrimination in the periphery. Further, psychophysical sensitivity to chromatic modulation has been observed to decrease at high temporal frequencies $(12 \mathrm{~Hz})$, whereas the relevant ganglion cells (midget ganglion cells for red-green modulation, small bistratified cells for blue-yellow modulation) show robust responses to high temporal frequency chromatic modulation. It has therefore been posited that low-pass filtering of chromatic information occurs at neural loci across the visual cortex.

In this thesis, I conducted three functional magnetic resonance imaging (fMRI) experiments on human subjects to investigate the cortical representation of peripheral as well as high temporal frequency chromatic information. In the first experiment, I employed retinotopic mapping methods to identify visual areas and to obtain detailed maps of visual field eccentricities. To ascertain the neural locus of peripheral color sensitivity loss, in the second experiment, I measured fMRI responses as a function of eccentricity in response to high cone-contrast chromatic, as well as luminance modulated circular grating stimuli. Furthermore, I studied the effect of spatial frequency on the eccentricity-dependent response.

The experimental findings in the primary visual cortex (V1) closely resemble retinal physiology, and imply that V1 is not associated with the psychophysical sensitivity loss. In addition, I observed a high degree of interaction between spatial frequency and retinal eccentricity. However, by accounting for the primary-cortical projection sizes (cortical magnification) by spatial frequency scaling at different eccentricities, it was possible to achieve an approximately even distribution of responses across eccentricity. 
When extending the analysis to the extrastriate cortex, it appears that the color-selective area V4 can be regarded as a neural substrate for the psychophysical sensitivity loss to redgreen colors in peripheral vision.

In the third experiment, I focused on ascertaining the characteristics of retinotopic visual areas in processing high temporal frequency chromatic information. To this aim, fMRI responses to high cone-contrast chromatic and luminance grating stimuli at various temporal frequencies were measured in both the lateral geniculate nucleus (LGN; the primary visual pathway's thalamic relay station) and cortical visual areas. Special M-scaled circular grating stimulus patterns were designed to eliminate the confounding effect of spatial frequency across visual eccentricity. The fMRI results provide clear evidence that high-temporal-frequency-chromatic information crosses LGN. On arrival in V1, however, blue-yellow information is subjected to low-pass filtering. This finding implies that a loss of psychophysical sensitivity to high temporal frequency blue-yellow information has a neural substrate as early as V1. There was no filtering of high temporal frequency red-green information in V1. Moreover, the data suggest that ventral and dorsal visual areas have distinct specialization for temporal frequency-dependent chromatic information. The ventral areas present with low-pass tuning characteristics, whereas the dorsal areas reveal robust responses to high temporal frequencies. In comparison to the other visual areas, responses in medial temporal area (MT) to luminance modulation show a strong amplification. Furthermore, MT responses increase with increasing temporal frequency, which is in line with MT's established role in luminance-mediated motion processing.

On the basis of the temporal frequency characteristic processing of luminance and chromatic information, it is proposed that visual areas can be hierarchically organized in clusters. A cluster containing the dorsal areas V3d \& V3a and, in combination with area MT, constitutes a functional network for the coding of high temporal frequency information. In contrast, another cluster comprising the ventral areas VP \& V4 constitutes a functional network for processing low temporal frequency chromatic information. Hence, it might provide a neural substrate for the psychophysical sensitivity loss to high temporal frequency chromatic information. These findings provide neurophysiological evidence for two behaviorally defined processing streams for motion that differ, mostly, in their temporal characteristics. 

6

References 
Abramov, I. \& GoRdon, J. (1977). Color vision in the peripheral retina II, Hue and saturation. Journal of the Optical Society of America 67, 202-206.

AbRAMOV, I., GORDON, J. \& CHAN, H. (1991). Color appearance in the peripheral retina: effects of stimulus size. Journal of the Optical Society of America A 8, 404-414.

Anderson, S. J., Mullen, K. T. \& Hess, R. F. (1991). Human peripheral spatial resolution for achromatic and chromatic stimuli: limits imposed by optical and retinal factors. Journal of Physiology 442, 47-64.

Anstis, S. \& CAVANAGH, P. (1983). A minimum motion technique for judging equiluminance. Academic Press, London.

Bach, M., Meigen, T. \& Strasburger, H. (1997). Raster-scan cathode-ray tubes for vision research--limits of resolution in space, time and intensity, and some solutions. Spatial Vision 10, 403-414.

BARLOW, H. B. (1986). Why have multiple cortical areas? Vision Research 26, 81-90.

Beauchamp, M. S., Haxby, J. V., Jennings, J. E. \& DeYoe, E. A. (1999). An fMRI version of the Farnsworth-Munsell 100-Hue test reveals multiple color-selective areas in human ventral occipitotemporal cortex. Cerebral Cortex 9, 257-263.

BILLMEYeR, F. W., JR. \& WebBeR, A. C. (1953). Three-dimensional color models constructed on the CIE and Munsell systems. Journal of the Optical Society of America 43, 69-70.

Brainard, D. H., Pelli, D. G. \& T., R. (2002). Display characterization. In: Encyclopedia of Imaging Science and Technology (Hornak J, ed), pp. 172-188.

Brainard, D. H., Roorda, A., Yamauchi, Y., Calderone, J. B., Metha, A., Neitz, M., Neitz, J., WILLIAMS, D. R. \& JACOBS, G. H. (2000). Functional consequences of the relative numbers of $L$ and $M$ cones. Journal of the Optical Society of America 17, 607-614.

Brewer, A. A., LiU, J. J., WAde, A. R. \& WANDell, B. A. (2005). Visual field maps and stimulus selectivity in human ventral occipital cortex (vol 8, pg 1102, 2005). Nature Neuroscience 8, 1411-1411.

Calkins, D. J., Schein, S. J., Tsukamoto, Y. \& Sterling, P. (1994). M and L cones in macaque fovea connect to midget ganglion cells by different numbers of excitatory synapses. Nature 371, 70-72.

Cavanagh, P., Tyler, C. W. \& Favreau, O. E. (1984). Perceived velocity of moving chromatic gratings. Journal of the Optical Society of America A 1, 893-899.

CAVonius, C. R. \& RobBins, D. O. (1973). Relationship between luminance and visual acuity of the rhesus monkey. Journal of Physiology 232, 501-511. 
Chatterjee, S. \& Callaway, E. M. (2002). S cone contributions to the magnocellular visual pathway in macaque monkey. Neuron 35, 1135-1146.

Chatterjee, S. \& Callaway, E. M. (2003). Parallel colour-opponent pathways to primary visual cortex. Nature 426, 668-671.

CHICKERING, K. D. (1969). Perceptual significance of the differences between CIE tristimulus values. Journal of the Optical Society of America 59, 986-990.

Cole, G. R., Hine, T. \& Mcllhagga, W. (1993). Detection mechanisms in L-, M- and S-cone contrast space. Journal of the Optical Society of America A 10, 38-51.

ConWAY, B. R. (2001). Spatial structure of cone inputs to color cells in alert macaque primary visual cortex (V-1). Journal of. Neuroscience. 21, 2768-2783.

ConWAY, B. R. \& LIVINGSTONE, M. S. (2006). Spatial and temporal properties of cone signals in alert macaque primary visual cortex. Journal of Neuroscience. 26, 10826-10846.

CotTARIS, N. P. \& De VAloIS, R. L. (1998). Temporal dynamics of chromatic tuning in macaque primary visual cortex. Nature 395, 896-900.

CoWeY, A. \& Rolls, E. T. (1974). Human cortical magnification factor and its relation to visual acuity. Experimental Brain Research 21, 447-454.

Crook, J. M., Lange-Malecki, B., Lee, B. B. \& ValberG, A. (1988). Visual resolution of macaque retinal ganglion cells. Journal of Physiology 396, 205-224.

Curcio, C. A., Kimberly, A. A., Sloan, K. R., Lerea, C. L., Hurley, J. B., Klock, I. B. \& Milam, A. H. (1991). Distribution and morphology of human cone photoreceptors stained with anti-blue opsin. Journal of Comparative Neurology 312, 610-624.

DACEY, D. M. (1993). The mosaic of midget ganglion cells in the human retina. Journal of Neuroscience13, 5334-5355.

DACEY, D. M. \& LEE, B. B. (1994). The 'blue-on' opponent pathway in primate retina originates from a distinct bistratified ganglion cell type. Nature 367, 731-735.

DANIELS, P. M. \& WhitTERIDGE, D. (1961). The representation of the visual field on the cerebral cortex in monkey. Journal of Physiology 159, 203-221.

DAW, N. W. (1968). Colour-coded ganglion cells in the goldfish retina: extension of their receptive fields by means of new stimuli. Journal of Physiology 197, 567-592.

de Monasterio, F. M., Schein, S. J. \& McCrane, E. P. (1981). Staining of blue-sensitive cone of the macaque retina by a fluorescent dye. Science $\mathbf{2 1 3}, 1278$. 
Demonasterio, F. M. \& SCHEIN, S. J. (1982). Spectral bandwidths of color-opponent cells of geniculocortical pathway of macaque monkeys. Journal of Neurophysiology 47, 214224.

DerRington, A. (2001). Vision: Why do colours fade at the edges? Nature 410, 886-887.

Derrington, A. M., Krauskopf, J. \& LenNie, P. (1984). Chromatic mechanisms in lateral geniculate nucleus of macaque. Journal of Physiology 357, 241-265.

DeRrington, A. M. \& LeNNIE, P. (1984). Spatial and temporal contrast sensitivities of neurones in lateral geniculate nucleus of macaque. Journal of Physiology 357, 219-240.

DESIMONE, R. \& SCHEIN, S. J. (1987). VISUAL PROPERTIES OF NEURONS IN AREA V4 OF THE MACAQUE - SENSITIVITY TO STIMULUS FORM. Journal of Neurophysiology 57, 835868.

Desimone, R., Schein, S. J., Moran, J. \& Ungerleider, L. G. (1985). Contour, color and shape analysis beyond the striate cortex. Vision Research. 25, 441-452.

DeValois, R. L., Abramov, I. \& Jacobs, G. H. (1966). Analysis of response patterns of LGN cells. Journal of the Optical Society of America 56, 966-977.

DeValois, R. L. \& JacoBs, G. H. (1968). Primate Color Vision. Science 162, 533-540.

DeYoe, A. \& VAN ESSEN, D. C. (1985). Segregation of efferent connections and receptive field properties in visual area V2 of the macaque. Nature $317,58-61$.

DeYoe, E. A., Bandettini, P., Neitz, J., Miller, D. \& Winans, P. (1994). Functional magnetic resonance imaging (FMRI) of the human brain. Journal of Neuroscience Methods 54, 171-187.

DeYoe, E. A., Carman, G. J., Bandettini, P., Glickman, S., Wieser, J., Cox, R., Miller, D. \& Neitz, J. (1996). Mapping striate and extrastriate visual areas in human cerebral cortex. Proceedings of the National Academy of Sciences USA 93, 2382-2386.

Dobkins, K. R. \& ALbRIGHT, T. D. (1995). Behavioral and neural effects of chromatic isoluminance in the primate visual motion system. Visual neuroscience 12, 321-332.

Dow, B. M. \& GouRAs, P. (1973). Color and spatial specificity of single units in Rhesus monkey foveal striate cortex. Journal of Neurophysiology 36, 79-100.

Engel, S., ZhANG, X. \& WANDelL, B. (1997a). Colour tuning in human visual cortex measured with functional magnetic resonance imaging. Nature 388, 68-71.

Engel, S. A., Glover, G. H. \& WANDell, B. A. (1997b). Retinotopic organization in human visual cortex and the spatial precision of functional MRI. Cerebral Cortex 7, 181-192. 
Engel, S. A., Rumelhart, D. E., Wandell, B. A., Lee, A. T., Glover, G. H., Chichilnisky, E. J. \& SHADLEN, M. N. (1994). fMRI of human visual cortex. Nature 369, 525.

FARNSWORTH, D. (1943). The Farnsworth-Munsell 100 hue and dichotomous tests for color vision Journal of the Optical Society of America 33, 568-578.

FELLEMAN, D. \& ESSEN, D. V. (1991). Distributed hierarchical processing in the primate cerebral cortex. Cerebral Cortex 1, 1-47.

Felleman, D. J. \& EsSEN, D. C. V. (1987). Receptive field properties of neurons in area V3 of macaque monkey extrastriate cortex. Journal of Neurophysiology 57, 889-920.

Ferrera, V. P., Nealey, T. A. \& Maunsell, J. H. (1994). Responses in macaque visual area V4 following inactivation of the parvocellular and magnocellular LGN pathways. Journal of Neuroscience 14, 2080-2088.

FitZPATRICK, D., ITOH, K. \& DiAmond, I. T. (1983). The laminar organization of the lateral geniculate body and the striate cortex in the squirrel monkey (Saimiri sciureus). Journal of Neuroscience 3, 673-702.

Fransson, P., Kruger, G., Merboldt, K. D. \& Frahm, J. (1998). Temporal characteristics of oxygenation-sensitive MRI responses to visual activation in humans. Magnetic Resonance in Medicine 39, 912-919.

GalBRAith, W. \& MARShALL, P. N. (1985). A survey of transforms of the CIE 1931 chromaticity diagram with some new non-linear transforms and histological illustrations of their utility. Acta Histochemica 77, 79-100.

GegenfurtneR, K. R. (2003). Cortical mechanisms of colour vision. Nature Reviews Neuroscience 4, 563-572.

GegenfuRtneR, K. R. \& HAWKen, M. J. (1996). Interaction of motion and color in the visual pathways. Trends in Neuroscience 19, 394-401.

Gegenfurtner, K. R., Kiper, D. C., Beusmans, J. M. H., Carandini, M., Zaidi, Q. \& Movihon, J. A. (1994). Chromatic properties of neurons in macaque MT. Visual Neuroscience 11, 455-466.

Gegenfurtner, K. R., Kiper, D. C. \& Fenstemaker, S. B. (1996). Processing of color, form, and motion in macaque area V2. Visual Neuroscience 13, 161-172.

GegenfurtneR, K. R., Kiper, D. C. \& LeVitT, J. B. (1997). Functional properties of neurons in macaque area V3. Journal of Neurophysiology. 77, 1906-1923.

Goodale, M. A. \& Milner, A. D. (1992). Separate Visual Pathways for Perception and Action. Trends in Neurosciences 15, 20-25. 
GoURAS, P. (1974). Opponent-colour cells in different layers of foveal striate cortex. Journal of Physiology 199, 533-547.

GRUESHOW, M. (2007). Chromatic responses in visual cortex - Effects of color, velocity and contrast, University of Magdeburg, Magdeburg.

GUR, M. \& SNODDERLY, D. M. (1997). A dissociation between brain activity and perception: Chromatically opponent cortical neurons signal chromatic flicker that is not perceived. Vision Research 37, 377-382.

haAse, A., Matthaei, D., Bartkowski, R., Duhmke, E. \& Leibfritz, D. (1989). Inversion recovery snapshot FLASH MR imaging. Journal of Computer Assisted Tomography 13, 10361040.

hansen, T., Pracejus, L. \& Gegenfurtner, K. R. (2009). Color perception in the intermediate periphery of the visual field. Journal of Vision $\mathbf{9}, 13$.

HaWken, M. J., Shapley, R. M. \& Grosof, D. H. (1996). Temporal-frequency selectivity in monkey visual cortex. Visual Neuroscience 13, 477-492.

Hawken, M. J., Shapley, R. M., Ringach, D. L., Sceniak, M. P., Johnson, E. N., Mareschal, I. \& HENRIE, J. A. (2001). Contrast gain as function of temporal frequency of macaque V1 neurons. Investigative Ophthalmology \& Visual Science 42, S726-S726.

HendRY, S. H. \& REID, R. C. (2000). The koniocellular pathway in primate vision. Annual Review of Neuroscience. 23, 127-153.

Henriksson, L., Nurminen, L., Hyvarinen, A. \& Vanni, S. (2008). Spatial frequency tuning in human retinotopic visual areas. Journal of Vision 8, 5 1-13.

Hofer, H., Carroll, J., Neitz, J., Neitz, M. \& Williams, D. R. (2005). Organization of the human trichromatic cone mosaic. Journal of Neuroscience 25, 9669-9779.

Horton, J. C., Landau, K., Maeder, P. \& Hoyt, W. F. (1990). Magnetic resonance imaging of the human lateral geniculate body. Archives of Neurology 47, 1201-1206.

HubeL, D. H. \& Wiesel, T. N. (1962). Receptive fields, binocular interaction, and functional architecture in the cat's visual cortex. Journal of Physiology 160, 106-154.

HubeL, D. H. \& Wiesel, T. N. (1968). Receptive fields and functional architecture of monkey striate cortex. Journal of Physiology 195, 215-243.

Huk, A. C., Dougherty, R. F. \& Heeger, D. J. (2002). Retinotopy and functional subdivision of human areas MT and MST. Journal of Neuroscience 22, 7195-7205. 
Molton, J. D., (2002). When the rainbow resembles the tricolour of France: The two subsystems of colour vision. (Eds) Rétine, cerveau et vision des couleurs, pages 3-17, Solal, Marseille, Solal, Marseille.

JIANG, Y., ZHOU, K. \& HE, S. (2007). Human visual cortex responds to invisible chromatic flicker. Nature Neuroscience 10, 657-662.

Kandel, E. R., Schwartz, J. H. \& Jessel, T. T. (2000). Principles of Neural Science. McGraw-Hill.

Kastner, S., O'Connor, D. H., Fukui, M. M., Fehd, H. M., Herwig, U. \& Pinsk, M. A. (2004). Functional imaging of the human lateral geniculate nucleus and pulvinar. Journal of Neurophysiology 91, 438-448.

KeLLY, D. H. (1983). Spatiotemporal variation of chromatic and achromatic contrast thresholds. Journal of the Optical Society of America 73, 742-750.

Kiper, D. C., Fenstemaker, S. B. \& Gegenfurtner, K. R. (1997). Chromatic properties of neurons in macaque area V2. Visual Neuroscience. 14, 1061-1072.

Kleinschmidt, A., Lee, B. B., Requardt, M. \& Frahm, J. (1996). Functional mapping of color processing by magnetic resonance imaging of responses to selective $\mathrm{P}$ - and $\mathrm{M}$ pathway stimulation. Experimental Brain Research 110, 279-288.

Krauskopf, J., Williams, D. R. \& HeEley, D. W. (1982). Cardinal directions of color space. Vision Research 22, 1123-1131.

KWAK, Y. \& MACDONALD, L. (2000). Characterisation of a desktop LCD projector. Displays 21, 179-194.

LEE, B. B. (1996). Receptive fields in primate retina. Vision Research 36, 631-644.

LEE, B. B. (2004). Paths to colour in the retina. Clinical and Experimental Optometry 87, 239248.

LEE, B. B. (2008). Neural models and physiological reality. Visual Neuroscience 25, 231-241.

Lee, B. B., Martin, P. R. \& Valberg, A. (1989). Nonlinear summation of M- and L-cone inputs to phasic retinal ganglion cells of the macaque. Journal of Neuroscience 9, 1433-1442.

Lee, B. B., Pokorny, J., Smith, V. C., Martin, P. R. \& Valberg, A. (1990). Luminance and chromatic modulation sensitivity of macaque ganglion cells and human observers. Journal of the Optical Society of America A 7, 2223-2236.

LEE, B. B., SUN, H. \& ZUCCHINI, W. (2007). The temporal properties of the response of macaque ganglion cells and central mechanisms of flicker detection. Journal of Vision 7, 1 116. 
LENNIE, P. (2000). Color vision: putting it together. Current Biology 10, R589-591.

Lennie, P., HaAke, P. W. \& Williams, D. R. (1991). The design of chromatically opponent receptive fields. In Computational models of visual processing. ed. LANDY, M. S. \& Movshon, J. A., pp. 71-82. MIT Press, Cambridge, MA, USA.

Lennie, P., Krauskopf, J. \& Sclar, G. (1990). Chromatic mechanisms in striate cortex of macaque. The Journal of Neuroscience 10, 649-669.

Leonova, A. \& Smithson, H. (2002). Book Review: Color Vision: From Genes to Perception. Edited by Karl R. Gegenfurtner and Lindsay T. Sharpe, Cambridge University Press, Cambridge, UK. 2001, xi + 492 pp., 59.95 (paperback). International Journal of Primatology 23, 1151-1152.

LeVenthal, A. G., Rodieck, R. W. \& Dreher, B. (1981). Retinal ganglion cell classes in the old world monkey: morphology and central projections. Science 213, 1139-1142.

LeVI, D. M., Klein, S. A. \& Aitsebaomo, A. P. (1985). Vernier acuity, crowding and cortical magnification. Vision Research 25, 963-977.

LeVI, D. M., KleIN, S. A. \& Aitsebaomo, P. (1984). Detection and Discrimination of the Direction of Motion in Central and Peripheral-Vision of Normal and Amblyopic Observers. Vision Research 24, 789-800.

LEVITT, J. B., KIPER, D. C. \& Movshon, J. A. (1994). Receptive fields and functional architecture of macaque V2. Journal of Neurophysiology. 71, 2517-2542.

Linden, D. E., Kallenbach, U., Heinecke, A., Singer, W. \& Goebel, R. (1999). The myth of upright vision. A psychophysical and functional imaging study of adaptation to inverting spectacles. Perception 28, 469-481.

LIU, J. \& WANDELL, B. A. (2005). Specializations for chromatic and temporal signals in human visual cortex. Journal of Neuroscience. 25, 3459-3468.

LiVINGstone, M. S. \& HubeL, D. H. (1984). Anatomy and physiology of a color system in the primate visual cortex. Journal of Neuroscience. 4, 309-356.

Logothetis, N. K. \& WANDelL, B. A. (2004). Interpreting the BOLD signal. Annual Review of Physiology 66, 735-769.

Lu, H., Basso, G., Serences, J. T., Yantis, S., Golay, X. \& van ZiJl, P. C. (2005). Retinotopic mapping in the human visual cortex using vascular space occupancy-dependent functional magnetic resonance imaging. Neuroreport 16, 1635-1640.

MANSFIELD, P. \& MAUdSLEY, A. A. (1977). Medical imaging by NMR. British Journal of Radiology 50, 188-194. 
Martin, P. R., Lee, B. B., White, A. J., Solomon, S. G. \& Ruttiger, L. (2001). Chromatic sensitivity of ganglion cells in the peripheral primate retina. Nature 410, 933-936.

Maunsell, J. H., Nealey, T. A. \& DePriest, D. D. (1990). Magnocellular and parvocellular contributions to responses in the middle temporal visual area (MT) of the macaque monkey. Journal of Neuroscience 10, 3323-3334.

MCKeEFRY, D. J. \& ZEKI, S. (1997). The position and topography of the human colour centre as revealed by functional magnetic resonance imaging. Brain 120 ( Pt 12), 2229-2242.

Merigan, W., Freeman, A. \& Meyers, S. P. (1997). Parallel processing streams in human visual cortex. Neuroreport 8, 3985-3991.

Merigan, W. H. \& MAUnSelL, J. H. (1993). How parallel are the primate visual pathways? Annual Review of Neuroscience. 16, 369-402.

Mirzajani, A., Oghabian, M. A., Riyahi-Alam, N., Saberi, H., Firouznia, K. \& Bakhtiary, M. (2006). Spatial frequency modulates the human visual cortical response to temporal frequency variation: an fMRI study. Conference Proceedings of IEEE Engineering in Medicine and Biology Society 1, 1032-1035.

Mollon, J. D. (1982). Color vision. Annual Review of Psychology 33, 41-85.

Molton, J. D. (1991). Uses and evolutionary origins of primate color vision. In Evolution of the eye and visual system, vol. 2. ed. CRONLY-DiLLON, J. R. \& GREGORY, R. L., pp. 306-319. MacMillan, London.

MUgleR, J. P., 3RD \& BROOKEMAN, J. R. (1990). Three-dimensional magnetization-prepared rapid gradient-echo imaging (3D MP RAGE). Magnetic Resonance in Medicine 15, 152-157.

MULLEN, K. T. (1985). The contrast sensitivity of human colour vision to red-green and blueyellow chromatic gratings. Journal of Physiology 359, 381.

MuLLEN, K. T. (1991). Colour vision as a post-receptoral specialization of the central visual field. Vision Research 31, 119-130.

Mullen, K. T., Dumoulin, S. O. \& Hess, R. F. (2008). Color responses of the human lateral geniculate nucleus: [corrected] selective amplification of S-cone signals between the lateral geniculate nucleno and primary visual cortex measured with high-field fMRI. European Journal of Neuroscience 28, 1911-1923.

Mullen, K. T., Dumoulin, S. O., McMahon, K. L., de Zubicaray, G. I. \& Hess, R. F. (2007). Selectivity of human retinotopic visual cortex to S-cone-opponent, L/M-coneopponent and achromatic stimulation. European Journal of Neuroscience 25, 491502. 
MuLLEN, K. T. \& KINGDOM, F. A. (1996). Losses in peripheral colour sensitivity predicted from "hit or miss" post-receptoral cone connections. Vision Resarch 36, 1995-2000.

MuLLEN, K. T. \& KINGDOM, F. A. (2002). Differential distributions of red-green and blue-yellow cone opponency across the visual field. Visual Neuroscience 19, 109-118.

Mullen, K. T., SAKuRAi, M. \& Chu, W. (2005). Does L/M cone opponency disappear in human periphery? Perception 34, 951-959.

Murphey, D. K., Yoshor, D. \& Beauchamp, M. S. (2008). Perception matches selectivity in the human anterior color center. Current Biology 18, 216-220.

NATHANS, J. (1999). The evolution and physiology of human color vision: insights from molecular genetic studies of visual pigments. Neuron 24, 299-312.

NeRI, D. F. (1990). Color CRT characterization presented solely in terms of the CIE system. Percept Mot Skills 71, 51-64.

Newton, J. R. \& ESKEW, R. T., JR. (2003). Chromatic detection and discrimination in the periphery: a postreceptoral loss of color sensitivity. Visual Neuroscience 20, 511-521.

NiCKERSO.D. (1969). History of Munsell Color System. Color Engineering 7, 42-\&.

NiCKERSON, D. (1940). History of the Munsell Color System and its scientific application. Journal of the Optical Society of America 30, 575-586.

Noorlander, C., Koenderink, J. J., den Ouden, R. J. \& Edens, B. W. (1983). Sensitivity to spatiotemporal colour contrast in the peripheral visual field. Vision Research 23, 111.

Ogawa, S., Tank, D. W., Menon, R., Ellermann, J. M., Kim, S. G., Merkle, H. \& Ugurbil, K. (1992). Intrinsic signal changes accompanying sensory stimulation: functional brain mapping with magnetic resonance imaging. Proceedings of the National Academy of Sciences USA 89, 5951-5955.

PEICHL, L. \& WASSLE, H. (1979). Size, scatter and coverage of ganglion cell receptive field centres in the cat retina. Journal of Physiology 291, 117-141.

REID, R. C. \& ShAPLEY, R. M. (1992). Spatial structure of cone inputs to receptive fields in primate lateral geniculate nucleus. Nature 356, 716-718.

REID, R. C. \& SHAPLEY, R. M. (2002). Space and time maps of cone photoreceptor signals in macaque lateral geniculate nucleus. Journal of Neuroscience 22, 6158-6175. 
Riecansky, I., Thiele, A., Distler, C. \& Hoffmann, K. P. (2005). Chromatic sensitivity of neurones in area MT of the anaesthetised macaque monkey compared to human motion perception. Experimental Brain Research 167, 504-525.

Rigby, H. S., Warren, B. F., Diamond, J., Carter, C. \& Bradfield, J. W. B. (1991). Color-Perception in Pathologists - the Farnsworth-Munsell 100-Hue Test. Journal of Clinical Pathology 44, 745-748.

Rodieck, R. W., Binmoeller, K. F. \& Dineen, J. (1985). Parasol and midget ganglion cells of the human retina. Journal of Comparative Neurology 233, 115-132.

ROVAMO, J., VIRSU, V. \& NASANEN, R. (1978). Cortical magnification factor predicts the photopic contrast sensitivity of peripheral vision. Nature 271, 54-56.

SCHEIN, S. J. \& DESIMONE, R. (1990). Spectral properties of V4 neurons in the macaque. Journal of Neuroscience. 10, 3369-3389.

SCHNAPF, J. L. \& BAYLOR, D. A. (1987). How photoreceptor cells respond to light. Scientific American 256, 40-47.

Sereno, M. I., Dale, A. M., Reppas, J. B., Kwong, K. K., Belliveau, J. W., Brady, T. J., Rosen, B. R. \& TOOTELL, R. B. (1995). Borders of multiple visual areas in humans revealed by functional magnetic resonance imaging. Science 268, 889-893.

Sereno, M. I., McDonald, C. T. \& Allman, J. M. (1994). Analysis of retinotopic maps in extrastriate cortex. Cerebral Cortex 4, 601-620.

SHAPLEY, R. (1990). Visual sensitivity and parallel retinocortical channels. Annual Review of Psychology. 41, 635-658.

Shapley, R. \& Perry, V. H. (1986). Cat and monkey retinal ganglion cells and their visual functional roles. Trends in Neurosciences 9, 229-235.

SinCiCH, L. C., PARK, K. F., Wohlgemuth, M. J. \& Horton, J. C. (2004). Bypassing V1: a direct geniculate input to area MT. Nature Neuroscience 7, 1123-1128.

Slotnick, S. D., Klein, S. A., CARney, T. \& Sutter, E. E. (2001). Electrophysiological estimate of human cortical magnification. Clinical Neurophysiology 112, 1349-1356.

SMithson, H. E. \& Mollon, J. D. (2004). Is the S-opponent chromatic sub-system sluggish? Vision Research 44, 2919-2929.

Solomon, S. G. \& LeNNIE, P. (2005). Chromatic gain controls in visual cortical neurons. Journal of Neuroscience 25, 4779-4792. 
Solomon, S. G. \& LeNNIE, P. (2007). The machinery of colour vision. Nature Reviews Neuroscience 8, 276-286.

SOlOMON, S. G., WHITE, A. J. \& MARTIN, P. R. (1999). Temporal contrast sensitivity in the lateral geniculate nucleus of a New world monkey, the marmoset Callithrix Jacchus. Journal of Physiology 517, 907-917.

Stockman, A. \& Sharpe, L. T. (2000). Tritanopic color matches and the middle- and longwavelength-sensitive cone spectral sensitivities. Vision Research 40, 1739-1750.

Stockman, A., Sharpe, L. T., Merbs, S. \& Nathans, J. (2000). Spectral sensitivities of human cone visual pigments determined in vivo and in vitro. Methods in Enzymology 316, 626-650.

Strasburger, H., Wustenberg, T. \& JANCKE, L. (2002). Calibrated LCD/TFT stimulus presentation for visual psychophysics in fMRI. Journal of Neuroscience Methods 121, 103-110.

Stromeyer, C. F., Lee, J. \& R.T. Eskew, J. (1992). Peripheral chromatic sensitivity for flashes: a post-receptoral red-green asymmetry. Vision Research 32, 1865-1873.

Sun, H., Smithson, H. E., ZAidI, Q. \& LeE, B. B. (2006). Do magnocellular and parvocellular ganglion cells avoid short-wavelength cone input? Visual Neuroscience. 23, 441-446.

SWANSON, W. H., PoKorny, J. \& SMITH, V. C. (1987). Effects of temporal frequency on phasedependent sensitivity to heterochromatic flicker. Journal of the Optical Society of America A 4, 2266-2273.

SzMAJDA, B. A. (2006). Subcortical Pathways for Colour Vision. The National Vision Research Institute of Australia and Department of Optometry \& Vision Sciences, PhD Thesis. The University of Melbourne, Melbourne.

TALAIRACH, J. \& TOURnAUX, P. (1988). Coplanar stereotaxic atlas of the human brain. . Thieme, New York

TAMRAZ, J. (1994). Neuroradiologic investigation of the visual system using magnetic resonance imaging. Journal of Clinical Neurophysiology 11, 500-518.

Thorell, L. G., DeValois, R. L. \& Albrecht, D. G. (1984). Spatial tuning of monkey V1 cells with pure color and luminance stimuli. Vision Research 24, 751-769.

Tootell, R. B., Hadjikhani, N. K., Vanduffel, W., Liu, A. K., Mendola, J. D., Sereno, M. I. \& Dale, A. M. (1998). Functional analysis of primary visual cortex (V1) in humans. Proceedings of the National Academy of Sciences USA 95, 811-817. 
Tootell, R. B., Reppas, J. B., Kwong, K. K., Malach, R., Born, R. T., Brady, T. J., Rosen, B. R. \& BeluIVEAU, J. W. (1995). Functional analysis of human MT and related visual cortical areas using magnetic resonance imaging. Journal of Neuroscience 15, 3215-3230.

Tootell, R. B., Silverman, M. S., Hamilton, S. L., De Valois, R. L. \& SWitkes, E. (1988). Functional anatomy of macaque striate cortex. III. Color. Journal of Neuroscience 8, 1569-1593.

TS'O, D. Y. \& GILBERT, C. D. (1988). The organization of chromatic and spatial interactions in the primate striate cortex. Journal of Neuroscience. 8, 1712-1727.

TYLER, J. E. \& HARDY, A. C. (1940). An analysis of the original Munsell Color System. Journal of the Optical Society of America 30, 587-590.

UngeRLEIDER, L. D. \& MISHKIN, M. (1982). Two cortical visual systems. In: Analysis of visual behaviour. MIT Press, Cambridge.

Vakrou, C., Whitaker, D., McGraw, P. V. \& McKeefry, D. (2005). Functional evidence for conespecific connectivity in the human retina. Journal of Physiology 566, 93-102.

Vanni, S., Henriksson, L., Vilkari, M. \& James, A. C. (2006). Retinotopic distribution of chromatic responses in human primary visual cortex. European Journal of Neuroscience 24, 1821-1831.

VIRSU, V., NÄSÄNEN, R. \& OSMOVIITA, K. (1987). Cortical magnification and peripheral vision. Journal of the Optical Society of America A 4, 1568-1578.

VIRSU, V. \& RoVAmo, J. (1979). Visual resolution, contrast sensitivity, and the cortical magnification factor. Experimental Brain Research 37, 475-494.

Wade, A., Augath, M., Logothetis, N. \& Wandell, B. (2008). fMRI measurements of color in macaque and human. Journal of Vision 8, 6 1-19.

Wade, A. R., Brewer, A. A., Rieger, J. W. \& Wandell, B. A. (2002). Functional measurements of human ventral occipital cortex: retinotopy and colour. Philosophical Transactions of the Royal Society of London Series B-Biological Sciences 357, 963-973.

WANDELL, B. A. (1999). Computational neuroimaging of human visual cortex. Annual Reviews of Neuroscience 22, 145-173.

Wandell, B. A., Poirson, A. B., Newsome, W. T., Baseler, H. A., Boynton, G. M., Huk, A., Gandhi, S. \& SHARPE, L. T. (1999). Color signals in human motion-selective cortex. Neuron 24, 901-909.

Warnking, J., Dojat, M., Guerin-Dugue, A., Delon-Martin, C., Olympieff, S., Richard, N., CHEHIKIAN, A. \& SEGEBARTH, C. (2002). fMRI retinotopic mapping--step by step. Neuroimage 17, 1665-1683. 
WÄSSLE, H. \& BOYCOTT, B. B. (1991). Functional architecture of the mammalian retina. Physiological Reviews 71, 447-480.

WATson, A. B. (1987). Estimation of local spatial scale. Journal of the Optical Society of America A 4, 1579-1582.

Watson, J. D., Myers, R., Frackowiak, R. S., Hajnal, J. V., Woods, R. P., Mazziotta, J. C., Shipp, S. \& ZEKI, S. (1993). Area V5 of the human brain: evidence from a combined study using positron emission tomography and magnetic resonance imaging. Cerebral Cortex $\mathbf{3}$, 79-94.

WERNER, J. S. Human colour vision 2: Colour appearance and cortical transformations.

White, A. J., Solomon, S. G. \& MARTiN, P. R. (2001). Spatial properties of koniocellular cells in the lateral geniculate nucleus of the marmoset Callithrix jacchus. Journal of Physiology 533, 519-535.

White, A. J. R., Goodchild, A. K., Wilder, H. D., Sefton, A. E. \& Martin, P. R. (1998). Segregation of receptive field properties in the lateral geniculate nucleus of a New-World monkey, the marmoset, Callithrix jacchus. Journal of Neurophysiology 80, 2063-2076.

Wiesel, T. \& HubEL, D. H. (1966). Spatial and chromatic interactions in the lateral geniculate body of the rhesus monkey. Journal of Neurophysiology 29, 1115-1156.

WYSZECKI, G. (1954). A graphical interpretation of a three-components theory of chromatic adaptation in terms of the CIE chromaticity diagram. Journal of the Optical Society of America 44, 787-792.

Yeh, T., Lee, B. B. \& KRemers, J. (1995). The temporal response of ganglion cells of the macaque retina to cone-specific modulation. Journal of the Optical Society of America A 12, 456-464.

ZEKI, S. (1983a). Colour coding in the cerebral cortex: the reaction of cells in monkey visual cortex to wavelengths and colors. Neuroscience $9,741-765$.

ZEKI, S. (1983b). Colour coding in the cerebral cortex: the responses of wavelength-selective and colour-coded cells in monkey visual cortex to changes in wavelength composition. Neuroscience 9 , 767-781.

ZeKI, S. \& MARINI, L. (1998). Three cortical stages of colour processing in the human brain. Brain 121, 1669-1685.

Zeki, S., Watson, J. D., Lueck, C. J., Friston, K. J., Kennard, C. \& Frackowiak, R. S. (1991). A direct demonstration of functional specialization in human visual cortex. Journal of Neuroscience 11, 641-649. 
Abbreviations 
3D

ANOVA

B

BOLD

CBF

CFF

CO

cpd

deg

EPI

FLASH

fMRI

G

GLM

$\mathrm{Hz}$

KC

LCD

L-cone

LGN

$\mathrm{MC}$

M-cone

$\mathrm{mm}$

MPRAGE

MRI

MT

PC

$\mathrm{R}$

ROI

$\mathrm{s}$

S-cone

TAL
Three-dimensional

Analysis of variance

Blue

Blood oxygenation level dependent

Cerebral blood flow

Critical flicker fusion

Cytochromoxidase

Cycles per degree

Degree

Echo planar imaging

Fast low angle shot

Functional magnetic resonance imaging

Green

General linear modeling

Hertz

Koniocellular

Liquid crystal display

Long-wavelength sensitive cone

Lateral geniculate nucleus

Magnocellular

Medium-wavelength sensitive cone

Millimeter

Magnetization prepared rapid gradient-echo sequence

Magnetic resonance imaging

Medial temporal

Parvocellular

Red

Region of interest

Seconds

Short-wavelength sensitive cone

Talairach 
TE

TR

V1

$\mathrm{V} 2 \mathrm{~d} / \mathrm{v}$

V3a

V3d

V4

VP

$V_{\lambda}$
Time to echo

Repetition time

Primary visual cortex (or striate cortex)

Visual area 2 dorsal/ventral

Visual area 3 accessory (a dorsal region)

Visual area 3 dorsal

Visual area 4 (ventral)

Ventral part of visual area V3

Luminosity function 

Acknowledgements 
First and foremost, I wish to express my sincere gratitude to Prof. Dr. Jens Frahm for giving me the opportunity to pursue doctoral studies in his vibrant research lab. I am very thankful to him for providing an excellent research facility, constant support, and encouragement.

It is with a deep sense of gratefulness I would like to acknowledge the finest supervision Prof. Dr. Barry Lee has offered me during the course of this thesis work. This work could not have been realized without the constant, companionable and stimulating guidance from him. Thank you Barry for introducing me to the colorful world of visual neuroscience! This has been the most inspirational phase of my life.

I am thankful to Prof. Dr. Stefan Treue, for providing me insightful comments, valuable suggestions and feedback at the thesis committee meetings.

I am deeply indebted to Prof. Dr. Hans Strasburger for his constant interest in my research work and for sharing his insights and amazing knowledge in vision science with me. Working with you has been a real treat Hans!

I would like to extend my sincere thanks to Dr. Klaus-Dietmar Merboldt - who from my day one in the lab has been very helpful in teaching me the know-how of MRI - for his key role in establishing the new set-up for vision experiments.

Very special thanks to:

Dr. Henry Lütcke, for introducing me to $\mathrm{fMRI}$ experimentation, data analysis

Dr. Tibor Auer, for his never ending patience, help in statistics, exciting discussions

Dr. Renate Schweizer, for her support, discussions and the pep talks

Dr. Sabine Hofer, for her patience in reading through the thesis, and feedback

Dr. Dirk Voit, for the know-how of MRI

Kurt Böhm, for technical and computational support

Sylke Wallbrecht, for her secretarial assistance

Lutz Präkelt, for assistance in installing the experimental set-up

All staff members of the Biomedizinische NMR Forschungs $\mathrm{GmbH}$

Dr. Hans-Christoph Nothdurft, for lending me the color vision test apparatus

Rev. Jephrin Monis, for his generosity, moral support

All my friends who have always been there for me through thick and thin

Finally, I would like to express a sense of my deepest gratitude to my parents, for their unconditional love, support, and blessings. Also, I would like to extend my warmest thanks to my grandmother, brother and his family, and relatives. 
9

Curriculum Vitae 
Dany V. D'Souza, в.E., M.Tech.

Zindel Str. 1

37073 Göttingen

Germany

Email: ddsouza@gwdg.de

\section{Personal details}

Date of Birth:

13.08.1980

Place of Birth:

Pilar-Karnataka, India

Nationality:

Indian

\section{Education}

2006-2009

PhD in Systems Neuroscience

University of Göttingen, Germany

Research lab: Biomedizinische NMR Forschungs $\mathrm{GmbH}$

am Max-Planck-Institut für biophysikalische Chemie

2004-2005

Research Student

Dept. of Medical Informatics

University of Heidelberg, Germany

2003-2004

Master Thesis

Dept. of Biology

Technical University of Kaiserslautern, Germany

2002-2004

M. Tech. in Biomedical Engineering

Manipal University, India

1998-2002

\section{B. E. in Biomedical Engineering}

Manipal University, India

\section{Professional experience}

03/2005-10/2005

\section{Research Assistant}

Dept. of Clinical Epidemiology

German Cancer Research Center, Heidelberg, Germany

\section{Conferences}

$09 / 2009$

Optical Society of America Fall Vision Meeting

Seattle, WA-USA

06/2008 Organization for human brain mapping

Melbourne, Australia

$09 / 2007$

Optical Society of America Fall Vision Meeting

Berkeley, CA-USA

05/2007

International Society for Magnetic Resonance in Medicine

Berlin, Germany 


\section{Published abstracts}

2008

D'Souza, D. V., Lee, B. B., \& Frahm, J. (2008). fMRI of chromatic and achromatic responses in human visual areas: Specializations for spatial \& temporal frequency. Neurolmage, 41(s1): S130. [Organization for Human Brain Mapping]

2007

D'Souza, D. V., Lee, B. B., \& Frahm, J. (2007). Do chromatic responses in V1 match retinal output or perceptual performance? [Abstract]. Journal of Vision, 7(15):60, 60a, http://journalofvision.org/7/15/60/, doi:10.1167/7.15.60

\section{Manuscripts in preparation}

2009

"Dependence of chromatic responses in human primary visual cortex on visual field eccentricity and spatial frequency of the stimulus - An fMRI study"

Dany V. D'Souza, Hans Strasburger, Jens Frahm \& Barry B. Lee

"Temporal frequency tuning of chromatic and luminance responses in human lateral geniculate nucleus and retinotopic cortical visual areas" Dany V. D'Souza, Tibor Auer, Hans Strasburger, Jens Frahm \& Barry B. Lee 